\title{
A MATRIX-BASED REGULARITY MEASURE FOR SYMBOLIC SEQUENCES
}

\author{
TREVOR WINE
}

\begin{abstract}
A set of statistics is developed for defining and determining the regularity of symbolic sequences. This is achieved by testing a given sequence against a template set with fixed asymptotic symbol proportions $p_{i}, \sum_{i} p_{i}=$ 1. The process centers on casting the sequence into matrix product form, and defining a parametrized probability distribution via the entrywise norms. The parameter allows varying the weighting between strict adherence to the template sequences, and a generalized Bernoulli randomness. The logarithms of the norms of the sequences under test are shown to satisfy a central limit theorem. This allows the assignment of z-scores, and rigorous comparison of the regularity between sequences of different types. The random vectors associated with the method are also found to have a well-defined entropy rate, leading to a definition of symbolic sequence homogeneity entropy. Numerical methods for estimating the entropy are also developed. Potential applications are explored, including time series and ergodic systems.
\end{abstract}

\section{Contents}

1. Introduction

1.1. Paper layout

2. Regularity distributions; binary alphabets and bit splicing, $p=1 / 2$

2.1. Two extremes at $p=1 / 2$ : fully regular and fully random

2.2. Parametrization between extremes

2.3. Meaning of intermediary parameter values, $\delta \in(0,1)$

3. Extension I: $p>1 / 2$, splicing

3.1. Development of template sequences, $p>1 / 2$

3.2. Some examples

4. Qualities of the probability distributions; random matrix theory

4.1. Random matrix theory in particular 15

4.2. Brute force Monte Carlo 20

4.3. Some example sequences; z-scores 21

5. Extension II: Pad-based methods 23

5.1. Padding method I: padding only 23

5.2. Padding method II: padding and splicing 25

5.3. Central limit theorem for padding 26

5.4. Examples for all three methods: splice-only, pad-and-splice, and pad-only

2020 Mathematics Subject Classification. 94A55, 60B20, 94A12, 37B10, 94A17.

Key words and phrases. sequence regularity, sequence discrepancy, random matrix products. 
6. Extension III: increasing the proportion spread; matrix dimensions $>d_{0}+d_{1}$

7. Extension IV: alphabets with more than 2 symbols 31

7.1. Simplices introduction 31

7.2. Matrix representations of simplex shifts 34

$\begin{array}{lll}\text { 7.3. } & \text { Block rows as graphs } & 36\end{array}$

7.4. Relations to sequence discrepancy and fairness 38

7.5. Graph properties and Christoffel extensions; cylinder graphs 42

7.6. Discrepancy examples 44

7.7. Regularity matrices for longer symbol alphabets 44

7.8. The central limit theorem and z-scores for longer symbol alphabets 46

7.9. Procedure for calculating z-scores 49

8. Distribution entropy 50

8.1. Entropy at the $\delta=0, \delta=1$ extremes; topological entropy on shifts

8.2. Entropy of homogeneity: stationary stochastic processes, entropy rate, mixing, and the AEP 54

8.3. Monte Carlo techniques for entropy 62

9. Properties of the measures 64

9.1. Forms of variance $\quad 65$

9.2. Effects of noise $\quad 66$

9.3. Displacing a given bit 66

9.4. Shuffling segments of fixed types 67

9.5. Simple periodic sequences; varying the inter-1 separation 70

9.6. Periodicity in general 70

9.7. Measures and definitions of regularity 71

$\begin{array}{ll}\text { 9.8. Contrasts with complexity measures } & 73\end{array}$

$\begin{array}{ll}\text { 10. Applications } & 73\end{array}$

10.1. Encoding a time series $\quad 73$

10.2. Heart rate data: z-scores and entropy of homogeneity 75

$\begin{array}{ll}\text { 10.3. The logistic map } & 78\end{array}$

$\begin{array}{ll}\text { 10.4. Sinai billiards } & 79\end{array}$

11. Discussion $\quad 80$

$\begin{array}{lr}\text { Appendix } & 84\end{array}$

$\begin{array}{lr}\text { References } & 86\end{array}$

\section{Introduction}

Studies of smoothness or evenness in the distribution of symbols in symbolic sequences include both measures of homogeneity, as well as associated methods of sequence generation. In the simplest case, with binary alphabets, generation methods often start with mechanical words, which one may think of as pixellated lines in $\mathbb{R}^{2}$. These may be further classified as either Sturmian or Christoffel words, depending on the rationality of line slope. Sturmian words are infinite, non-periodic binary words of lowest factor complexity, and obey a specific smoothness condition: they are said to be balanced. Balanced words obey limits in the variation of symbol 
counts across sub-words of a given length (see Section 3.1 for definitions of a factor and the balance property). Even at this most basic level, Sturmian words have applications in resource allocation, from the work of Hajek, [21], in single server queuing systems and beyond [1], and have served a role in foundational studies of dynamical systems [40].

For longer alphabets of more than two symbols, approaches for creating relatively smooth sequences include hypercubic billiards ([60], Section 6), episturmian words [14], and synching and related methods which build a homogeneous word over alphabets of more than two letters by augmenting regular binary sequences with additional symbols ([53, 1]; and see again [60], which contains yet other methods).

Scheduling theory has its own, though related approaches to constructing and measuring smooth symbolic sequences, notably sequence discrepancy and various definitions of sequence fairness [28]. These methods too are primarily concerned with minimizing the error in symbol proportion off a fixed ideal on blocks of various lengths (see Section 7.4 for a formal definition of sequence discrepancy).

Areas of application for such research include manufacturing, with for instance product rate variation (PRV) and apportionment problems [64], dynamical systems [12], discrete event systems ([60], Section 7), and especially information technology, for example in communication networks and CPU resource allocation (stride scheduling) [61], among others. A good introduction is provided in [28].

The main result of this paper is the development of a framework to precisely quantify degrees of symbolic sequence regularity: to determine for example how 'close' the sequence 010010101100 is to the 'fully regular' sequence 101010101010 , at a finer scale than basic edit distance. In general, sequences of the second type will be referred to as fully regular template sequences, or template sequences for short. The method is applicable to sequences over any finite alphabet, $\mathcal{A}=\left\{\alpha_{1}, \ldots, \alpha_{k}\right\}$, and will weigh the sequences under test against template sequences, $T=(r)_{n \in \mathbb{N}}$, with asymptotic rational symbol proportions $p_{i} \in \mathbb{Q}$ :

$$
\forall i \in\{1, \ldots, k\}, \quad \lim _{m \rightarrow \infty} \frac{1}{m} \sum_{j=1}^{m} \chi_{\left\{\alpha_{i}\right\}}\left(r_{j}\right)=p_{i},
$$

where $\chi$ is the characteristic function.

The degree of permitted local variation in the symbol proportions of the template sequences will be left adjustable. For any associated set of asymptotic proportions, $\left\{p_{1}, \ldots, p_{k}\right\}$, a nested hierarchy of template sets is constructed, $R_{1} \subseteq R_{2} \subseteq \ldots$, where the local proportion for any $\alpha$ on blocks of fixed length has an allowed variation that increases monotonically in the $R_{i}$ index. This variation is directly related to the 'balancedness' of the sequences of the template set, or, equivalently, their sequence discrepancy. For example, in the binary case, with $p=1 / 2$, we have strictest templates $R_{1}=\{\overline{01}, \overline{10}\}$ (where the overline indicates infinite repetition of the word), or we may loosen the local variation in proportion, to allow template sequences such as $\overline{000111}$ (in some higher-indexed $R_{i}$ ).

The template sequences are directly derived from corresponding matrix product forms. As a simple example, any products formed by the matrix pair, $\left\{\left[\begin{array}{ll}0 & 1 \\ 0 & 0\end{array}\right],\left[\begin{array}{ll}0 & 0 \\ 1 & 0\end{array}\right]\right\}$, must strictly alternate, what amounts to a matrix representation of binary template sequences $1010 \ldots$ and $0101 \ldots$. The template matrix sets are paired via a parameter with simple generalized Bernoulli diagonal matrices, 
$p_{i} \mathbb{I}$, resulting in a probability distribution formed by the norms of matrix products over all sequences of a given length. The resulting ranking is tied to the test sequence's proximity to any one of the template sequences, where the string distance metric is roughly an aggregate Levenshtein distance taken over all subsequences, and/or padded 'supersequences' of the test sequence.

The probabilities themselves, as the norms of matrix products, obey a central limit theorem, which allows the assignment of z-scores. This distributional aspect of the measure has an advantage over discrete measures of sequence smoothness, such as sequence balance, or sequence discrepancy, which, at least for rational symbol proportions, may have high degeneracies in their values; that is, many different sequences of a given length can possess the same value of the measure. This broader distributional approach allows a finer-scale discernment in degrees of sequence homogeneity.

In the binary symbol case, the template classes are simply prefixes of powers of cyclic rotations of Christoffel words. They may also be modeled as constrained walks on a 1-dimensional volume. A natural extension to $k$-symbol alphabets is derived by increasing the dimension of the volume, specifically to $(k-1)$-simplices. The simplex-generated template classes themselves appear to be new. We have with them, in compact form, via the associated template sequence matrices or their corresponding graphs, a complete expression of all possible sequences at a given level of homogeneity. The matrix template set is further derivable from a recursive algorithm, given in the Appendix.

In addition, independent of the central limit theorem and z-scores, the random vector probability distributions arising from the norms of the matrix products themselves give rise to a distributional entropy. By a stationarity property associated with a stochastic process closely related to the random vectors' distributions, for $m$ sufficiently large, the limiting average entropy, or entropy rate, is found to exist. This allows the definition of the symbolic sequence homogeneity entropy. The related stationary process in the single block row case is also found to be ergodic and therefore obeys an asymptotic equipartition property (AEP). This provides further insight into the distribution of norms of matrix products over sequences of fixed length, beyond what the central limit theorem provides, and offers the potential for coding and compression theorems. The homogeneity entropy can be considered to be narrower in scope and more tailored to homogeneity than the usual entropy-based sequence statistics (ApEn, SampEn, etc.).

1.1. Paper layout. The paper is organized as follows. Section 2 develops the basic method under the simplest case: a binary alphabet with equiprobable symbols, $P(0)=P(1)=1 / 2$. The sequence under test is cast into a product of 2 by 2 matrices, as above: each matrix the parametrized sum of a regularity template component (a nilpotent, or shift part), and a Bernoulli component (a diagonal part). A binomial expansion of an arbitrary product of the two-component matrices is shown to be a weighted sum over all possible template-regular subsequences of the test sequence. The process can be characterized in terms of an aggregate bitsplicing string metric. The combination of a nilpotent shift template component and a purely diagonal generalized Bernoulli component lies at the heart of the process throughout the paper. 
Section 3 involves the first generalization, to any rational binary symbol proportion $p \in[1 / 2,1) \cap \mathbb{Q}$. A few example sequences are given, along with their computed probability scores, highlighting important characteristics of the bit-splicing method.

Section 4 is an excursion into random matrix theory. This allows proving the logarithms of the matrix norms, the norms themselves already interpretable as probabilities, converge weakly as a function of sequence length to a normal distribution under a central limit theorem for the products of random matrices. Utilizing estimates for the Lyapunov exponent and variance for the associated random matrix product chains, computed via Monte Carlo methods, this allows the assignment of $z$-scores to sequences of any $p$, and of sufficient length.

For the next extension, in Section 5, we expand the underlying string metric between the sequence under test and the template sequences from bit splicing to bit padding. The approach of Section 2 is modified accordingly. By the end of this section we have a 'full' set of measures on binary sequences with rational symbol proportions $p \in[1 / 2,1)$, under the two basic underlying string metric operationspadding and splicing, alone or in combination.

Section 6 loosens the restrictions on template sequence regularity, what amounts to increasing the maximum allowed discrepancy, or symbol proportion variability on local scales, for the template sequences. This is accomplished by a simple increase in matrix dimension.

Section 7 extends the regularity distributions to alphabets of $k>2$ symbols. This centers around the creation of 'simplicial shift operators' and their associated vectors, which generalize the one-dimensional action of the shift operators from the binary case to those in $k-1$ dimensions. The template sequences, under a minimal volume condition, may be viewed as natural $k$-dimensional geometric extensions of Christoffel words.

In Section 8, the entropy of the distributions under arbitrary alphabets is examined. A symbolic sequence regularity-, or homogeneity entropy is defined. We also tailor an existing random matrix Monte Carlo resampling technique to allow the numerical estimation of the entropy and entropy rate of the distributions represented by the (normalized) matrix norms.

Section 9 considers the developed statistics as sequence regularity measures. Relevant characteristics are examined further, and advantages and disadvantages relative to certain existing sequence regularity measures, such as statistical variance and non-linear complexity measures, are weighed.

In Section 10 the method is applied to human heart rate data, both through z-score analysis and by estimating heart rate homogeneity entropy, as well as to the logistic map, and the Sinai billiard.

\section{Regularity distributions; binary alphabets and bit splicing, $p=1 / 2$}

We begin with the simplest case, $p=P(0)=1 / 2$, with the lowest amount of local variation in the template sequences.

2.1. Two extremes at $p=1 / 2$ : fully regular and fully random. The method involves constructing probability distributions at the fully regular matrix extreme, and at the Bernoulli, fully random matrix extreme. Once accomplished, a parameter is introduced to linearly interpolate between the two extremes. 
The template matrices comprising the fully regular extreme in this case are, as mentioned in the introduction,

$$
S_{0}=\left[\begin{array}{ll}
0 & 1 \\
0 & 0
\end{array}\right], S_{1}=\left[\begin{array}{ll}
0 & 0 \\
1 & 0
\end{array}\right] .
$$

Any products of this matrix pair will always correspond to regular sequences $0101 \ldots$ and $1010 \ldots$.

At the fully random, Bernoulli extreme we simply have,

$$
P_{0}=\left[\begin{array}{ll}
p & 0 \\
0 & p
\end{array}\right], P_{1}=\left[\begin{array}{ll}
q & 0 \\
0 & q
\end{array}\right] .
$$

In aim of inducing a probability distribution over binary sequences of length $m$ at both extremes via the matrix norm, we next consider normalization constants. That is, we require a $c_{N}$ at each extreme such that,

$$
\sum_{i_{1}, \ldots, i_{m}=0,1} c_{N}\left\|A_{i_{1}} \cdots A_{i_{m}}\right\|_{e n}=1
$$

where $A$ is a notational stand-in for the $S_{i}$ at one extreme and $P_{i}$ at the other, and where for convenience we've chosen the entrywise 1-norm (i.e. the sum of the absolute values of the matrix entries, here denoted by $\left.\|\cdot\|_{e n}\right)$.

We can take advantage of the fact that since the $A_{i}$ matrices have non-negative entries, by properties of the entrywise 1-norm,

$$
\left\|\left(A_{0}+A_{1}\right)^{m}\right\|_{e n}=\left\|A_{0} \cdots A_{0}\right\|_{e n}+\left\|A_{0} \cdots A_{1}\right\|_{e n}+\ldots+\left\|A_{1} \cdots A_{1}\right\|_{e n},
$$

(i.e. the entrywise norm commutes with the binomial sum). The normalization constant then is simply

$$
c_{N}=\frac{1}{\left\|\left(A_{0}+A_{1}\right)^{m}\right\|_{e n}} .
$$

Noting that since both $S_{0}+S_{1}$ and $P_{0}+P_{1}$ are (doubly) stochastic, the entrywise norm of any power of either sum is simply the dimension of the matrices, 2 , giving $c_{N}=1 / 2$ in both cases. This produces the desired probability distribution at both extremes.

At the purely random extreme, the probability of finite binary sequence $b \in$ $\{0,1\}^{m}$ equals that of the associated outcome of a sequence of i.i.d. Bernoulli trials: $p^{(b)_{0}} q^{(b)_{1}}$, where $(b)_{i}$ counts the number of $i$-bits in the sequence. At the purely regular extreme, the sequences $0101 \ldots 1$ and $1010 \ldots 0$ are equiprobable with $P(b)=1 / 2$, while the probability of all other sequences is 0 .

We note that there is some freedom in choosing the weighting between the two $S_{i}$ template matrices. Throughout the paper, the template matrices will always be binary. This has the effect of providing equal weighting, in terms of the entrywise 1-norm, to each of all possible infinite product chains associated with a template matrix product. This is made clear in Section 3.1, where an equivalence between surviving sequences of matrix template products and the number of available constrained random walks on a line segment is developed. 
2.2. Parametrization between extremes. Now we introduce parameter $\delta \in$ $[0,1]$ to linearly interpolate between the two matrix-sequence extremes:

$$
\begin{aligned}
& M_{0}=\delta P_{0}+(1-\delta) S_{0}=\left[\begin{array}{cc}
(1 / 2) \delta & (1-\delta) \\
0 & (1 / 2) \delta
\end{array}\right] \\
& M_{1}=\delta P_{1}+(1-\delta) S_{1}=\left[\begin{array}{cc}
(1 / 2) \delta & 0 \\
(1-\delta) & (1 / 2) \delta
\end{array}\right] .
\end{aligned}
$$

When $\delta=1$, we have the pure probability case, and when $\delta=0$, we have the fully regular case. To have valid probability distributions when $\delta \neq\{0,1\}$, we need the correct normalizing constant. Since $M_{0}+M_{1}$ remains doubly stochastic for all $\delta \in[0,1]$, it follows immediately that $c_{N}=1 / 2$ throughout.

Given the simplicity of construction, the cases where $\delta$ is not at the extremes should produce something like a hybrid probability distribution, negotiating between the pure probability on the one hand and the rigidity of the fully regular sequences on the other. To ensure values $\delta \in(0,1)$ produce something meaningful, we'll take a look at this next.

2.3. Meaning of intermediary parameter values, $\delta \in(0,1)$. For a given binary sequence's matrix product, $M_{0} M_{1} M_{1} \cdots M_{0}$, we'll decompose each $M_{i}$ as $\delta r_{i} \mathbb{I}+(1-\delta) S_{i}$ where $\mathbb{I}$ is the two-by-two identity matrix, $r_{0}=p, r_{1}=q$, and the $S_{i}$ shift matrices are defined as above.

For some further notation, given a binary sequence $S$ of length $m$, denote a given subsequence of $S$ of length $k$ by $Q(k)$, and $Q^{c}(k)$ its complement (i.e. the subsequence formed from $S$ by removing elements of subsequence $Q(k)$ ). For example,

$$
S=100001010111101 ; Q(9)=100101010 ; Q^{c}(9)=001111 .
$$

Let $X_{i}$ be an element in a binary-indexed set of matrices or scalars, $\left\{X_{0}, X_{1}\right\}$. Then let $\left(X_{i}\right)_{Q(k)}$ denote the product of $X_{i}$ corresponding to subsequence $Q(k)$. Note that $\left(X_{i}\right)_{Q(m)}=\left(X_{i}\right)_{Q^{c}(0)}=\prod_{Q(m)} X_{i}$. (For another example, let $S=010$, and select a particular $Q(2)=01$ (chosen from $\{01,10,00\}$ ), then $Q^{c}(2)=0$, and $\left(S_{i}\right)_{Q(2)}=S_{0} S_{1}$.) Further, let $\left\{Q_{j}^{\prime}(k)\right\}_{j}$ be the set of only those $k$-long subsequences of $S$ whose $S_{i}$ product is nonzero, i.e. only index the subsequence $Q(k)$, by $Q_{j}^{\prime}$, if $\left(S_{i}\right)_{Q(k)} \neq 0$. We have that $\left(S_{i}\right)_{Q_{j}^{\prime}(1)}, j=1,2, \ldots$, indexes all nonzero 1-fold matrix products derived from length-1 subsequences of $S,\left(S_{i}\right)_{Q_{j}^{\prime}(2)}, j=1,2, \ldots$, indexes all nonzero 2 -fold products, etc.

Now expand the given matrix product in powers $\delta$ and $(1-\delta)$. Following the above notation,

$$
\begin{aligned}
M_{0} M_{1} & M_{1} \cdots M_{0} \\
& =\left(\delta p \mathbb{I}+(1-\delta) S_{0}\right)\left(\delta q \mathbb{I}+(1-\delta) S_{1}\right) \cdots\left(\delta p \mathbb{I}+(1-\delta) S_{0}\right) \\
& =\delta^{m}\left(r_{i}\right)_{Q^{\prime c}(0)} \mathbb{I}+\delta^{m-1}(1-\delta) \sum_{j}\left(r_{i}\right)_{Q_{j}^{\prime c}(1)}\left(S_{i}\right)_{Q_{j}^{\prime}(1)}+\ldots \\
& +\delta(1-\delta)^{m-1} \sum_{j}\left(r_{i}\right)_{Q_{j}^{\prime c}(m-1)}\left(S_{i}\right)_{Q_{j}^{\prime}(m-1)}+(1-\delta)^{m}\left(S_{i}\right)_{Q^{\prime}(m)} .
\end{aligned}
$$

A given $\delta^{m-k}(1-\delta)^{k}$ term then has as its coefficient the sum of all and only the surviving matrix products of length $k$, each summand weighted by the ( $m-k$ long) $Q_{j}^{\prime c}(k)$ complement of $p$ and $q$ factors. 
Each nonzero summand of the coefficient of $\delta^{m-k}(1-\delta)^{k}$ then has split the sequence into a fully regular subsequence of length $k$, the surviving product of the $S_{i}$, and a purely binomial $p, q$ power term formed from the complementary subsequence. This essentially splits the summand between the two probability extremes. In a general statistical sense, considering all the summands in a respective sum in (2.3) at a given $k$ value, we should see the largest net probability mass accrue to sequence splittings that (a) have a $k$-long fully regular subsequence (the fully regular extreme), and (b) have in the subsequence complement approximately $p(m-k)$ zeros (the binomial extreme).

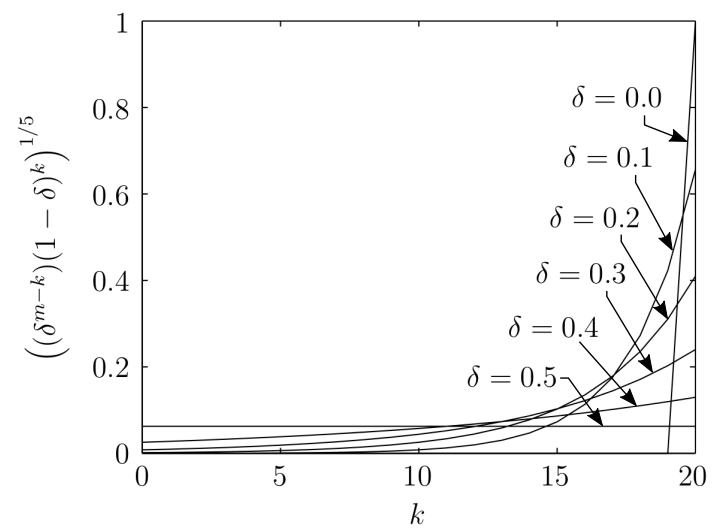

FIGURE 1. Delta polynomial terms, $m=20$ (under $\sqrt[5]{ }$ scaling for clarity)

Given this binomial formulation in $\delta$, consider the effect of changing $\delta$ on the collection of $\delta^{m-k}(1-\delta)^{k}$ coefficients. Consulting Figure 1 , at $\delta=0$, all the weight is on the last coefficient $(k=m)$. As $\delta$ is increased, more and more weight is shifted to $\delta$ polynomial terms at lower values of $k$, until $\delta=0.5$, where all powers of $\delta$ receive equal weighting. (The figure is left/right symmetric as $\delta$ is increased from 0.5 to 1 .)

Some further terminology will be helpful for understanding the role of $\delta$. Given a binary sequence, let a splice be defined as the removal of one bit from the sequence. Now given two sequences, $A$ and $B$, we'll say $A$ covers $B$ if, after zero or more splices from $A$, we can make $A=B$ (e.g. 01001 covers 1001 by splicing its first bit). Given a sequence $S$ of length $m$, and a fully regular sequence $F$ of length $\leq m$, we'll say that $S$ is subregular to $F$ if $S$ covers $F$. When this is the case, let the difference in lengths between $S$ and $F,|S|-|F| \geq 0$, be defined as the subregular cover distance between $S$ and $F$. We note the subregular cover distance qualifies as a simple string metric, and may be regarded as a subcase of the more general Levenshtein distance, which is a count of the minimal number of insertions, deletions, and/or substitutions required to morph one word into another.

The choice of $\delta$ then may be viewed as modulating the weighting assigned to each subregular cover distance, $m-k$, relative to the input sequence, according to the curves in Figure 1. Smaller $\delta$ s will emphasize lower subregular cover distances (i.e. favoring relatively long fully regular subsequences covered by the given sequence 
$S$ ), while higher $\delta$ s will favor higher subregular cover distances, which amounts to emphasizing shorter fully regular subsequences covered by $S$.

Let $\left\{F_{i}\right\}_{i}$ denote the set of all fully regular subsequences covered by $S$. Define the order of a covering of a given $F_{i}$ as the ordinal position in the least-to-most ordering of the set $\left\{|S|-\left|F_{i}\right|\right\}_{i}$. A covering of first order is then all $F_{i}$ covered by $S$ such that $|S|-\left|F_{i}\right|$ is at its minimum. A covering of second order is all $F_{i}$ covered by $S$ such that $|S|-\left|F_{i}\right|$ is next-to-lowest. And so on. When $\delta<0.5$, from (2.3) we see that the largest $\delta^{m-k}(1-\delta)^{k}$ term corresponding to a non-zero $\sum\left(r_{i}\right)\left(S_{i}\right)$ is when $m-k$ equals the subregular cover distance associated with the first order covering. The associated value $k$, the length of the longest subsequence covered by $S$, then provides a first order estimate of the relative magnitude of the matrix norm.

Note that for higher subregular cover distances, when considering all shorter subsequences of $S$ whose products survive, the number of such products is reasonably maximized by balancing the fine-grained regularity of $S$ in a $p$-proportion of 0 s. Statistically, the closer to a $p$ portion the fine-grained regularity of $S$ is (i.e. the closer it is to fully regular), the more surviving $S_{i}$ matrix products of length $k$ there should be (it may be thought of as sampling all $k$-long subsequences from $S$, which under random sampling should produce the most surviving products when the proportion of 0 's is closest to $p$ on the smallest scale possible). But this is balanced by the complementary $p$ and $q$ power factors in a somewhat complicated relationship; whenever $p>q$, as in the next generalization, of Section 3, 0s will be favored. As an example, we'd expect there to be more surviving products derived from all possible subsequences of length 3 from the sequence 0101001010100 than from the sequence 0000011110000, but if we took $p=1-\epsilon$, and therefore $\mathrm{P}(1)=q=\epsilon$, the $p$ and $q$ power factors could overwhelm the higher surviving subsequence count in the first sequence. Also note that if we consider sufficiently short subsequences, then all may survive (in this case all subsequences of length 1 will survive). In any case, there is some 'tunability' available with the parameter $\delta$, to alter according to the desired properties of the regularity measure.

It is certainly the case that for a given sequence, if $S$ contains one or more fully regular subsequences of length $\approx m$, the corresponding higher order terms in (2.3) (terms associated with $\delta^{m-k}(1-\delta)^{k}, k \approx m$ ) will stand out well above the mean probability of $1 / 2^{m}$, provided $\delta$ is small. This suggests the parametrization works as intended, allowing the sorting of fully regular and nearly fully regular sequences by their having higher probability values assigned, over sequences less-well matched to the templates (provided $\delta$ is sufficiently small). Making $\delta$ too small on the other hand, risks over-penalizing sequences that are almost fully regular.

\section{Extension I: $p>1 / 2$, splicing}

It is straightforward to extend the developments of Section 2 to $p \in(1 / 2,1) \cap \mathbb{Q}$ (recalling $p$ is the expected density of 0 's in the sequence: $p \approx \# 0^{\prime} s / m$ ). Given a rational $p \in(1 / 2,1)$, in lowest terms, $p=d_{1} / n$, let $d_{0}=n-d_{1}\left(\operatorname{gcd}\left(d_{0}, d_{1}\right)=1\right)$. Note by the requirement on the range of $p, d_{0}<d_{1}$. (By symmetry, of course, for the sequences we only need be concerned with $p \in[1 / 2,1)$, i.e. we can always swap 1s for $0 \mathrm{~s}$.)

3.1. Development of template sequences, $p>1 / 2$. For any matrix, denote diagonal position $d$ by $d=0$ for the main diagonal, $d>0$ for diagonals $d$ above 
and to the right of the main diagonal, and $d<0$ for diagonals $d$ below and to the left of the main diagonal. Construct the nilpotent $n$ by $n$ matrix shift pair, $S_{0}$ and $S_{1}$, as follows. Let $S_{0}$ be defined by a single diagonal of 1's at position $d_{0}$, and let $S_{1}$ be defined by a single diagonal of 1 's at position $-d_{1}$. For example, if $p=2 / 3$, $n=3, d_{0}=1, d_{1}=2$, and we have,

$$
S_{0}=\left[\begin{array}{lll}
0 & 1 & 0 \\
0 & 0 & 1 \\
0 & 0 & 0
\end{array}\right], S_{1}=\left[\begin{array}{lll}
0 & 0 & 0 \\
0 & 0 & 0 \\
1 & 0 & 0
\end{array}\right] .
$$

We'll now examine in general what sequences are considered fully regular.

Consider the action of the shift matrices on symbolic vector,

$$
v_{\text {test }}=\left[\begin{array}{c}
x_{1} \\
x_{2} \\
\vdots \\
x_{n}
\end{array}\right] \text {. }
$$

The left-action of $S_{0}$ on $v_{\text {test }}$ is to shift all elements up by $d_{0}$, while the left-action of $S_{1}$ on $v_{\text {test }}$ is to shift all elements down by $d_{1}$. This shift action may be viewed in two associated ways throughout the paper. The first is as shifts of a line segment of length $n-1$, placed exactly within the boundary interval on $\mathbb{R}$ of $[0, n-1]$, and with the stipulation that after a given shift any component of the segment lying outside the boundary is sheared off. The other way of viewing the shifts is as a random walk in one dimension on a horizontal segment of length $n-1$, with $n$ allowed positions indexed from $0:\{0, \ldots, n-1\}$. In all cases, only two types of shifts or steps are permitted: those to the left of size $d_{0}$, and to the right of size $d_{1}$.

Claim 1. Given $S_{0}$ and $S_{1}$ as above, the entrywise 1-norm of any given product of $S_{i}$ 's corresponds to the number of possible starting positions for the associated random walk on a segment $l$ with $n$ positions. The walk consists of step sizes to the left of magnitude $d_{0}$, and to the right of magnitude $d_{1}$, where the steps are taken in the reverse order of the product (i.e. the rightmost matrix in the product counts as the first step).

Proof. The left-action of $S_{0}$ (resp. $S_{1}$ ) on the test vector $v_{t e s t}$ is to shift the rows of $v_{\text {test }}$ up (resp. down) by $d_{0}$ (resp. $d_{1}$ ) rows. The remaining non-zero elements in the vector are exactly those starting positions that allow the walk to remain on segment $l$ after such a step. Composing this result with another $S_{i}$ now reflects all valid starting positions that remain on the segment after two such steps, and so on. We see the number of remaining (non-zero) indices on the test vector after $m$ steps is equal to the number of starting positions for that step sequence that allow it to stay on $l$. Noting that the entrywise 1-norm of any matrix product (of matrices with non-negative entries) is equal to the sum of elements of the vector resulting from the left-action of the product on $v=[1, \ldots, 1]^{\top}$, completes the proof.

A third way of looking at allowed shift actions that preserve a nonzero entrywise norm is via Christoffel words, which are a form of mechanical words. A mechanical word may be constructed geometrically by considering a line $l$ in $\mathbb{R}^{2}$ with respect to an integer lattice. At each coordinate grid line $x=j, j \geq 0$, we record the lattice point just below (resp. above) $l$. This produces a sequence of points, $P_{1}, P_{2}, \ldots$, with $x$ coordinates $0,1,2, \ldots$. We assign a binary sequence to $\left(P_{i}\right)$ by considering the line 


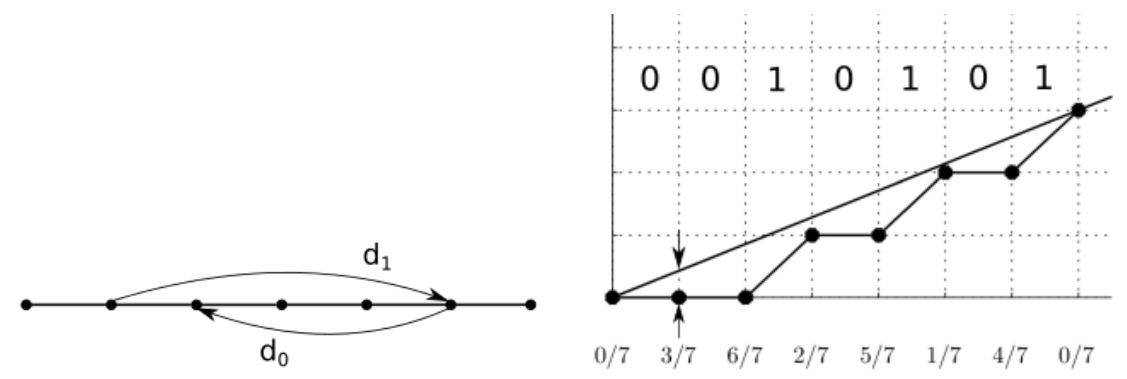

FIGURE 2. matrix shifts under $n=7, d_{0}=3, d_{1}=4$ as a constrained random walk, and as a Christoffel word

segment connecting $P_{i}$ to $P_{i+1}$ : if it is horizontal, assign a 0 ; if it is diagonal, assign a 1 . The resulting binary sequence is termed the lower (resp. upper) mechanical word associated with $l$. Mechanical words are also known as digitized straight lines.

If $l$ has irrational slope, the mechanical word is then a Sturmian word. Otherwise the slope of $l$ is rational, and the binary sequence is necessarily periodic. If line $l$ passes through the origin and is of positive slope, $m=p / q$ in lowest terms, then the lattice points just below (resp. above) the segment connecting $(0,0)$ and the point $(p, q)$ is termed the lower (resp. upper) Christoffel path corresponding to $m$. The binary sequence assigned to the path is called the lower (resp. upper) Christoffel word. See Figure 2.

Intuitively, in the context of sequence smoothness, Christoffel words appear to possess an optimal degree of regularity. In the field of combinatorics on words, this is formalized in the following way. Given a word $w$, a factor of $w$ is any contiguous string chosen from $w$ (a substring). A word is balanced if any two factors of the same length differ in a given symbol count by at most 1 . Infinite sequences formed by powers of Christoffel words, $w^{\infty}$, as well as Sturmian words, are balanced [60, 53]. A symbol count difference of 1 is the best we can do for words of two or more symbols. We link such maximally regular words to the $S_{i}$ template sequences with the following claim,

Claim 2. Let $w$ be the Christoffel word associated with the plot $y=\left(d_{0} / n\right) x$, $x \in[0, n]$, and let $\sigma_{j}(w), j \in[0,|w|-1]$, denote the cyclic permutation of $w$ that results from placing the first $j$ bits last. Then all and only the surviving products of the $S_{i}$ are prefixes of $\left(\sigma_{j}(w)\right)^{\infty}$, over all $j$.

Proof. This can be seen from comparing the restricted random walk associated with the $S_{i}$ shifts above, with the fractional portions between line $l$ and its (lower) approximating path. See Figure 2. The numerators of the fractional distances correspond exactly to a walk that remains on the segment with $n$ positions, $\{0, \ldots, n-1\}$, with steps to the right of length $d_{1}$, and to the left of length $d_{0}$. We note the fraction's numerators are simply generated via $k d_{0} \bmod n, k \in[0, n-1]$, $\operatorname{gcd}\left(n, d_{0}\right)=1$, and therefore cover all points on the segment exactly once. Since $d_{0}+d_{1}=n$, for each step there is only one choice in direction to remain on the segment at any point. This means there are no other $n$-long walks but those of the $\sigma_{j}(w)$. The extension to any length as a prefix of some $\left(\sigma_{j}(w)\right)^{\infty}$ follows immediately. 
These allowed $S_{i}$ products then form the $\left(n, d_{0}\right)$-regular binary sequence templates, and may be considered maximally regular under the balance property.

As mentioned in Claim 1, there are instances when products of length $<n$ have entrywise norms $>1$, reflecting multiple possible starting points, which reflects a prefix of multiple possible fully regular sequences. In the $d_{0}=3, d_{1}=4$ example, for instance 010 will have norm 3 , indicating it's a prefix to exactly 3 regular sequences.

Following the same development for the pure probability extreme, and the $\delta$ interpolation parameter, from the section on $p=1 / 2$, we have for the general case, $p \in[1 / 2,1), p=d_{1} / n$, the following sparse matrix pair,

$$
M_{0}=\left[\begin{array}{cccccc}
p \delta & 0 & \cdots & (1-\delta) & & \\
0 & \ddots & & & \ddots & \\
& & & & (1-\delta) \\
& & & & 0 \\
& & & & 0 & p \delta
\end{array}\right], M_{1}=\left[\begin{array}{ccccc}
q \delta & 0 & & \\
0 & \ddots & & \\
\vdots & & & \\
(1-\delta) & & & \\
& \ddots & & \\
& & (1-\delta) & 0 & 0 \\
& & & &
\end{array}\right]
$$

with $d_{0}$ and $d_{1}=n-d_{0}$ the diagonal offsets, and the associated fully regular sequences determinable as above. Probabilities are extracted for sequences of length $m$ by taking the entrywise 1-norm of the respective $M_{0}, M_{1}$ matrix product, and multiplying by the normalizing constant, which, as with the $p=1 / 2$ case, by double stochasticity throughout is $c_{N}=1 / n$.

3.2. Some examples. Some examples at this point will be helpful. Taking $p=$ $4 / 5, m=20$, the following are some representative sequences and the logarithms of their probability values (i.e. $\left.\log \left(c_{N}\left\|M_{i_{m}} \cdots M_{i_{1}}\right\|_{\text {en }}\right)\right)$ at two different $\delta$ s.

For $\delta=0.6$ :

$\begin{array}{ccc} & \text { sequence } & \log (\mathrm{P}(\mathrm{s})) \\ s_{1} & 000000000000100000000000000110 & -11.683 \\ s_{2} & 001000010000000100000101000001 & -14.288 \\ s_{3} & 000110000001000010000100100101 & -17.492\end{array}$

Sequence $s_{1}$ does not look even close to the fully regular sequence $\overline{10000}$ (and cyclic rotations) for this $p$ value. But the $p, q$ terms that weigh the spliced $S_{i}$ sequences skew results in favor of $0 \mathrm{~s}(P(0) \geq P(1))$, especially when shorter fully regular sequences are emphasized by higher $\delta$ values, as (2.3) suggests. This is further emphasized by sequences $s_{2}$ and $s_{3}$, which look quite regular, but actually receive a lower score, primarily because they have fewer 0s.

For $\delta=0.1$ :

$\begin{array}{lcc} & \text { sequence } & \log (\mathrm{P}(\mathrm{s})) \\ s_{4} & 100000001000010000010100010000 & -12.729 \\ s_{5} & 000010100010000011000001000000 & -14.985 \\ s_{6} & 000010100010000011000011110000 & -21.854 \\ s_{7} & 000000000110000110000010110010 & -29.690 \\ s_{8} & 100000000000000000001001011100 & -48.613\end{array}$

At this lower $\delta$ value, note sequence $s_{4}$ 's closeness to the $p=4 / 5$ regular sequence of $\overline{10000}$, while the lowest probability goes to the last sequence, $s_{8}$, with an obvious 
imbalance of $0 \mathrm{~s}$ and $1 \mathrm{~s}$. Sequence $s_{6}$ shows the splicing nature of the processit doesn't seem very regular, but with 6 splices it could be fully regular (as a foreshortened sequence), giving it its somewhat high probability value. Sequence $s_{5}$ can also be fully regular in just 6 splices, and the higher proportion of 0 s helps increase its probability over the one below, the $p, q$ weighting having an effect even at this relatively low value of $\delta$. Sequence $s_{7}$ suffers from the relatively large number of splices required to make it regular, from the initial run of $0 \mathrm{~s}$, to the grouped $1 \mathrm{~s}$. To further underscore how this distribution favors splicing, consider the following three sequences, of length $m=100$, at $p=4 / 5$, and their probability scores at $\delta=0.1$ :

$$
\begin{array}{cc}
\text { sequence } \mathrm{s}(\mathrm{m}=100) & \log (\mathrm{P}(\mathrm{s})) \\
\overline{10000} & -11.772 \\
\overline{100000} & -24.838 \\
\overline{1000} & -83.097
\end{array}
$$

The fully regular sequence is first. The middle sequence has 1s separated by exactly 5 zeros, and the last by exactly 3 zeros. Both the second and last would seem to be close to fully regular, but note the penalty on the last sequence for being so poorly suited for splicing. The second sequence does much better, because it requires far fewer splices to achieve full regularity.

Though this attaches some information to binary sequences, we'd like to know more about the distribution of the probability values themselves, so that sequence probabilities can be meaningfully compared to one another. This is explored next.

Remark. At this point if the reader is only interested in the development of homogeneity entropy, they may consider skipping Sections 4.1 through 4.3, which show the distributions obey a central limit theorem, allowing z-scores. The entropy development does not depend on the central limit theorem. Section 5 may then be read up through 5.2, on sequence padding. All of Sections 6 and 7 may be read, which develop larger proportion spreads and longer alphabets, with the exception of 7.8 , which again involves the central limit theorem and z-scores. The entropy is then developed in Section 8. Sections 10.1 and 10.2 then demonstrate an application for the homogeneity entropy, an analysis of heart rate data.

\section{Qualities of the probability distributions; random matrix theory}

For any sequence of length $m$, and any $p \in[1 / 2,1) \cap \mathbb{Q}$, we'd like to know something about the distribution of probability values, i.e. what is the distribution of the normalized entrywise 1-norms of summands of $\left(M_{0}+M_{1}\right)^{m}$.

In the $p=1 / 2,2$ by 2 case, from (2.2), note a $\delta / 2$ may be factored out, and the assignment $z=2(1-\delta) / \delta$ made to give

$$
M_{0}=\frac{\delta}{2}\left[\begin{array}{ll}
1 & z \\
0 & 1
\end{array}\right], M_{1}=\frac{\delta}{2}\left[\begin{array}{ll}
1 & 0 \\
z & 1
\end{array}\right] .
$$

Then the $m$-fold matrix products may be reformulated as $(\delta / 2)^{m}$ times a product of the $z$ matrices. Note that $\left[\begin{array}{ll}1 & z \\ 0 & 1\end{array}\right]^{k}=\left[\begin{array}{cc}1 & k z \\ 0 & 1\end{array}\right]$, and similarly for its transpose. Assuming the binary string's leftmost bit is a 0 , and writing $a_{0}$ as the number of leading 0 's, $a_{1}$ as the subsequent number of 1's, and so forth (so that e.g. 00011 is written as $\left[a_{0}, a_{1}\right]=[3,2]$ ), such products may be written in 
terms of continuants, depending on the terminating bit (see [20]). If given a sequence of bit runs $\left(a_{0}, \ldots, a_{m-1}\right)$, we denote $K\left(i_{a}, i_{b}\right), i_{a} \leq i_{b}$ as the continuant $K\left(a_{i_{a}} z, a_{i_{a}+1} z, \ldots, a_{i_{b}} z\right)$, then, for the matrix products, if the sequence ends

$$
\text { in } 0,=\left[\begin{array}{ll}
K(1, m-2) & K(1, m-1) \\
K(0, m-2) & K(0, m-1)
\end{array}\right] ; \text { in } 1,=\left[\begin{array}{cc}
K(0, m-1) & K(0, m-2) \\
K(1, m-1) & K(1, m-2)
\end{array}\right] \text {. }
$$

If the leftmost bit is a 1 , then we can simply invert all the bits, and use the result just above, taking the continuant matrix flipped about both the main diagonal and anti-diagonal. After including the outer $(\delta / 2)^{m}$ factor, this gives a complete formulation of the 2 by 2 products in terms of continuants.

The continuant formulation arises directly from a fundamental relation to continued fractions. If $\left[a_{0} z, \ldots, a_{m-1} z\right]$ represents a continued fraction in the common notation, then it is expressible as $K(0, m) / K(1, m)$. In the general $z$ case, we have Stieltjes fractions [33], and in the case $z=1$, we have ordinary continued fractions with integer terms [20]. In fact the matrix products in this case have entries corresponding to numerators and denominators of ancestral fractions of the end of the matching $\mathrm{R} / \mathrm{L}$ path (coding 0 as $\mathrm{L}$, and 1 as $\mathrm{R}$ ) in the Stern Brocot tree (see [20]). This special $z=1$ case is also fundamental to Farey fraction spin chains [26], and in topological mixing [51].

Though reformulating in terms of continuants does express 2 by 2 products in familiar terms, it does not tell us much about their general distribution. In fact, describing the general behavior of products of all but the simplest of matrices chosen from a discrete or continuous set poses significant challenges, especially if analytical results are desired.

At minimum, one of the most common values associated with random matrix products, which holds under a very general set of conditions, is the asymptotic growth rate of the norm as $m \rightarrow \infty$. It is termed the top, or primary Lyapunov exponent, and denoted here by $\lambda$. I.e. if $S_{m}$ is the random variable that takes on any of the possible $m$-fold matrix products, then

$$
\lim _{m \rightarrow \infty} \frac{1}{m} \log \left\|S_{m}\right\|
$$

exists and is almost surely equal to $\lambda$, independent of the choice of norm. But finding the Lyapunov exponent also tends to resist analytical approaches.

Most of the existing work in the general case of random products of 2 by 2 matrices involves finding or estimating the top Lyapunov exponent. In the particular case of the $M_{i}$, in fact [51] finds a way to algorithmically derive bounds on the Lyapunov exponent for the 2 by $2 z$-matrix pair above, at any value of $z$ in the appropriate range $(z>0)$.

Chassaing et al. [10] also work with asymptotic products of $z$-matrices, in the special case of $z=1$. They take the projection $(a x+b) /(c x+d)$ of the matrix product, $\left[\begin{array}{ll}a & b \\ c & d\end{array}\right]$, and link the distribution to continued fractions on $[0,1]$, which follows a Denjoy-Minkowski measure. Other results in the general 2 by 2 case include [59] which computes (analytically) an exact Lyapunov exponent for a simple 2 by 2 matrix pair associated with a random Fibonacci sequence; [2] which numerically computes Lyapunov exponents for several simple 2 by 2 matrix pairs; [34] which looks at properties of products of 2-dimensional matrices in context of random continued fractions of a certain type; as well as [5, 45, 31], all of which consider 
Lyapunov exponents primarily or in part. The literature indicates the difficulties of analytical methods even in the simple 2 by 2 case, and for results on the distribution of the $M_{i}$ products in the general case, we'll turn to classical probability analogs in random matrix theory.

Before we do there is one area of salvage. Asymptotic analytical results are attainable for the $M_{i}$, for all $p$, as $\delta \rightarrow 1$. Consider that for $\delta \approx 1$ we may write,

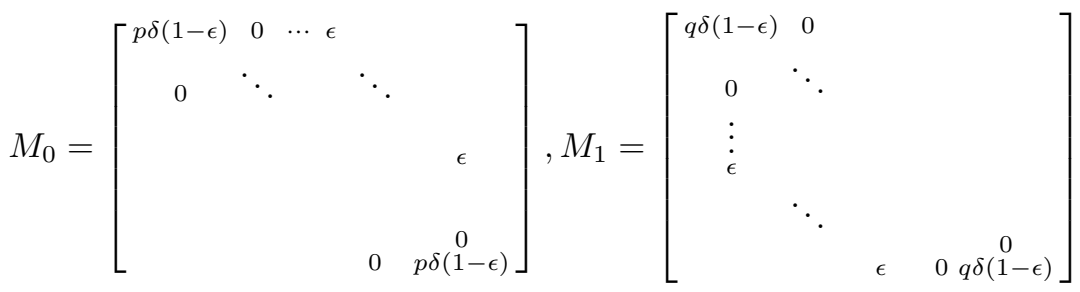

By equation (2.3), it follows that for any given $m$-fold product, the leading $\delta^{m}$ term will dominate; all other $\delta$ terms will have matrices with elements of order $\epsilon$. This means we can decompose all products as an approximation to a binomial distribution: we expect the values of $\left\|S_{m}\right\|_{e n} / n$ to cluster around values $\left\{p^{k} q^{m-k}\right\}_{k=0, \ldots, m}$, with respective frequency $\left(\begin{array}{c}m \\ k\end{array}\right)$. Consider the Gaussian approximation to the binomial coefficients (making the variance explicit),

$$
\left(\begin{array}{c}
m \\
k
\end{array}\right) \approx \frac{2^{m}}{\sqrt{2 \pi(m / 4)}} e^{-(x-m / 2)^{2} /(2 m / 4)} .
$$

If we apply $\log$ scaling to obtain even intervals on $k$ of $\log \left(p^{k} q^{m-k}\right)$, we shift the mean to the midpoint between $m \log q$ and $m \log p(q \leq p)$, and the variance is changed under the corresponding rescaling $x^{\prime}=(\log p-\log q) x$ to give the following mean and variance for the Gaussian approximation to the values of $\log \left(\left\|S_{m}\right\|_{e n} / n\right)$ as $\delta \rightarrow 1$ :

$$
\mu \approx \frac{m(\log p+\log q)}{2} ; \sigma^{2} \approx \frac{m(\log p-\log q)^{2}}{4} .
$$

Figure 3 contains a plot of the normalized norms, $\left\|S_{m}\right\|_{e n} / n$, at $p=3 / 5$, with $m=9$ and $\delta=0.85$. The lower plot is the norms sorted in increasing order, and the staircase (dashed line) corresponds to the expected binomial values. The upper plot is with the domain lexicographically ordered $(000000000,000000001, \ldots)$. The self-similarity of the resulting plot arises from the regular distribution of binaries of a given number of 0's (resp 1's) throughout this increasing binary ordering.

In general, away from $\delta=1$, the plots of the product norms get rather irregular. Figure 4 shows how the adherence to the Gaussian starts to decay as $\delta$ is lowered from 1 . With $\delta=0.1$, the fully regular sequences start to emerge with relatively high norms. (Note that at $p=3 / 5$, the fully regular sequences are prefixes of wordstrings $S_{r} S_{r} \ldots$, with $S_{r}$ equal to a cyclic rotation of 10100.) The other sequences have relatively low norms (probabilities).

4.1. Random matrix theory in particular. To obtain distributional information on the normalized $M_{i}$ products, $\left\|S_{m}\right\|_{e n} / n$, we might hope that in spite of the matrices' lack of commutativity, at least for large $m$ some of the asymptotic results from classical probability theory might apply, such as the central limit theorem. In fact, they do, in essence determining the distribution (of the norms, treated as a random variable) of a distribution (the norms viewed as a probability distribution in and of themselves, the random variable being the $m$-long binary sequences). Since 

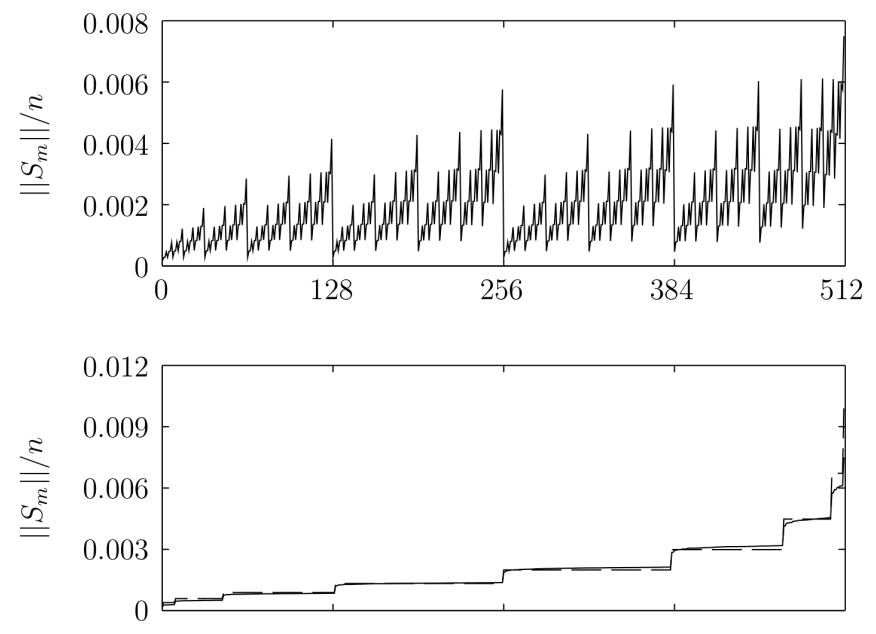

FiguRE 3. binary lexicographical ordering (top) vs. norms sorted in increasing order (bottom) at $m=9, p=3 / 5, \delta=0.85$; dashed line on bottom plot shows binomial $p^{k} q^{m-k}$ staircase
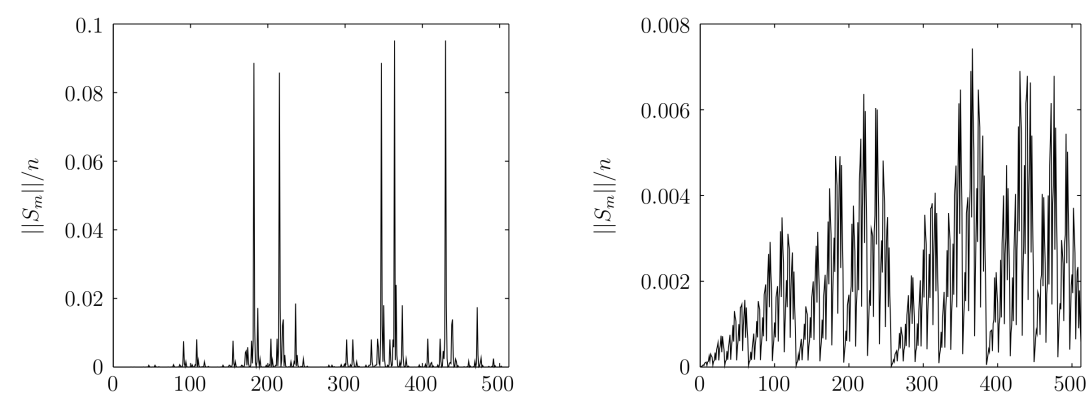

FIGURE 4. binary lexicographical ordering at $m=9, p=3 / 5$, for $\delta=0.1$, at left, and $\delta=0.5$, at right

the variance of $\log \left(c_{N}\left\|S_{m}\right\|_{e n}\right)$ is unchanged as a function of normalization constant $c_{N}$, and the mean is merely shifted by amount $\log \left(c_{N}\right)$, we will often drop the $c_{N}$ factor unless specifically discussing the probability distribution $P(S)=c_{N}\|S\|_{e n}$.

Let $V=\mathbb{R}^{n}$, and $G=G L(n, \mathbb{R})$. Let $\mu$ be a Borel probability measure with support $F \in G$, and $\Gamma_{\mu}$ be the semigroup spanned by $F$.

Given a sequence $\left\{Y_{m}, m \geq 1\right\}$ of i.i.d. $n$ by $n$ random matrix variables drawn from $F$, denote random matrix product $Y_{m} \ldots Y_{1}$, as above, by $S_{m}$. In particular, at this point we'll be interested in two discrete distributions. The first is,

$$
\mu_{e}=(1 / 2) \delta_{M_{0}}+(1 / 2) \delta_{M_{1}}
$$

i.e. a two-element discrete distribution, with support $\left\{M_{0}, M_{1}\right\}$, where $M_{0}$ and $M_{1}$ can occur with equal probability $1 / 2$. This will simply produce the distribution of 
the log of the random product norms. We'd also like a distribution that allows us to focus mostly on norms of sequences with the proportion of 0 's we're likely to put under test (those with a proportion of 0's at or close to $p$ ). This is conveniently easy to do, we just change $\mu$ to favor $M_{0}$ by a probability equal to its internal probability value, $p$ (assigning $M_{1}$ its complement, $q$ ):

$$
\mu_{p}=p \delta_{M_{0}}+q \delta_{M_{1}} .
$$

In discerning properties of the random product's norms, we see immediately the expectation value of the norm of $m$-fold products under $\mu_{e}$ is $\mathbb{E}\left(\left\|S_{m}\right\|_{e n}\right)=n / 2^{m}$. This follows from

$$
\sum_{i_{1}, \ldots, i_{m}=0,1} \frac{1}{2^{m}}\left\|M_{i_{1}} \cdots M_{i_{m}}\right\|_{e n}=\frac{1}{2^{m}}\left\|\left(M_{0}+M_{1}\right)^{m}\right\|_{e n}=\frac{n}{2^{m}}
$$

by commutativity of the entrywise norm, and stochasticity of the two-matrix sum, as above. To obtain information on the distribution of the values of $\left\|S_{m}\right\|_{e n}$ in general, at least asymptotically for $m \gg 1$, random matrix theory in this case provides insight into the behavior of the logarithms of the norms. We'll see this includes a central limit theorem for matrix products over either of the $\mu$ distributions.

First a basic result of random matrix theory, touched on above, on the log of the norm under certain rather loose conditions. It is credited to Furstenberg and Kesten [15]:

Theorem 1. Let $S_{m}=Y_{m} \cdots Y_{1}$, where $Y_{i}$ are i.i.d. random matrix variables with distribution $\mu$. If $\mathbb{E}\left(\log ^{+}\left\|Y_{1}\right\|\right)<\infty$ (for function $\left.f, f^{+} \equiv \max \{f, 0\}\right)$, with $\|\cdot\|$ denoting any matrix norm,

$$
\lim _{m \rightarrow \infty} \frac{1}{m} \log \left\|S_{m}\right\|
$$

exists and is equal to $\lambda$ with probability 1 , where

$$
\lambda=\lim _{m \rightarrow \infty} \frac{1}{m} \mathbb{E}\left(\log \left\|S_{m}\right\|\right) .
$$

The distributions $\mu_{e}$ and $\mu_{p}$, and the associated $M_{i}$, clearly meet the conditions of the theorem. The value $\lambda$, as mentioned, is the top Lyapunov exponent. Notice by definition of the equivalence of norms $\|.\|_{1}$ and $\|.\|_{2}$ that since $\log \|A\|_{1}$ differs from $\log \|A\|_{2}$ by at most an additive constant, $c$, and since $\lim _{m \rightarrow \infty} m^{-1} \mathbb{E}\left(\log \left\|S_{m}\right\|+\right.$ $c)=\lim _{m \rightarrow \infty} m^{-1} \mathbb{E}\left(\log \left\|S_{m}\right\|\right), \lambda$ is independent of the choice of norm.

Next is a main theorem on the applicability of the central limit theorem. Since when $\delta=0$ the distributions reduce to the trivial case of $n$ fully regular sequences each of probability $1 / n$, any time the central limit theorem is considered, we'll assume $\delta>0$.

The exact requirements on the matrices in the support of $\mu$ can vary. In the more general cases, strong irreducibility of $\Gamma_{\mu}$ is often a requirement $[8,30,18,6,7]$, a condition which for one guarantees separation of the two topmost Lyapunov exponents. Furstenberg, [15], proves a central limit theorem under the condition the entries of all matrices in the support of $\mu$ have strictly positive entries.

In all our cases, we are dealing with matrices with merely non-negative entries. Let $Z$ denote the semigroup of matrices of $G$ whose entries are non-negative, and whose every row and column contains at least one strictly positive element. Let positive cone $Z^{\circ}$ denote the subsemigroup, $Z^{\circ} \subset Z$, whose matrices' entries are strictly positive. The following theorem is derived directly from that of Hennion 
in [23], and will be used extensively. It relies on a condition that guarantees the random matrix products, beyond some minimal product length $m_{0}$ (dubbed the stopping time), will have strictly positive entries with probability one.

Theorem 2. Suppose $Y_{i}$ are i.i.d. random matrix variables with distribution $\mu$, with all matrices in the support of $\mu$ members of semigroup $Z$. Let $\lambda$ be the top Lyapunov exponent. Assume

$$
P\left(\bigcup_{j \geq 1}\left[Y_{j} \cdots Y_{1} \in Z^{o}\right]\right)>0 .
$$

Define the stopping time, $T=\inf \left\{m: m \geq 1, S_{m} \in Z^{\circ}\right\}$. Then under any matrix norm,

$$
\frac{1}{\sqrt{m}} 1_{[T \leq m]}\left(\log \left\|S_{m}\right\|-m \lambda\right)
$$

converges weakly to a centered normal distribution.

Proof. The result follows easily from [23], Theorem 3. Lemma 3.1 of [23] uses the $\bigcup_{j \geq 1}$ positivity condition to ensure the stopping time is finite. Theorem 3 of [23] states the central limit theorem result in terms of the projective action, $\log \left\langle y_{m}, S_{m} x_{m}\right\rangle$, where $\left(x_{m}\right)$ and $\left(y_{m}\right)$ are arbitrary sequences of unit vectors with non-negative components. Assigning $x_{m}=y_{m}=[1, \ldots, 1]^{\top} / \sqrt{n} \forall m$, we see the result applies to the matrix entrywise norm as well, and under equivalence of norms to any matrix norm, proving the theorem.

To show the $M_{i}$ meet the condition of the theorem, we first note a set of nonnegative matrices $K$ is irreducible if it fixes no common invariant subspace. This is equivalent to the statement that for every $i, j$, there is some matrix product $k_{l} \cdots k_{1}, k_{q} \in K$, such that its $i, j$ th entry is greater than zero (see for example $[46])$. We have the claim,

Claim 3. The set of matrices $\left\{S_{i}\right\}_{i}$ is irreducible.

Proof. This follows from first noting a finite set of matrices is irreducible iff their sum is irreducible. Then note by construction (see (3.1)), since $n=d_{0}+d_{1}, S_{0}+S_{1}$ is a cyclic permutation matrix (one that fixes no standard basis element $\hat{e}_{j}$ ). The claim follows.

This allows proof of the central limit theorem for the $M_{i}$ :

Corollary 1. Let $Y_{i}$ be random matrix variables under the discrete distributions $\mu_{e},(4.1)$, or $\mu_{p},(4.2)$, with values in $\left\{M_{0}, M_{1}\right\}$. Then,

$$
\frac{1}{\sqrt{m}}\left(\log \left\|S_{m}\right\|_{e n}-m \lambda\right)
$$

converges weakly to a centered normal distribution.

Proof. We show the $M_{i}$ meet the eventual positivity condition of Theorem 2. Recall the $M_{i}$ are of form $a \mathbb{I}+b S_{i}, a, b>0$. Irreducibility of $\left\{S_{0}, S_{1}\right\}$ by Claim 3 ensures that for every $i, j$ there is some $k_{l}<\infty$ and some product $S_{i_{k_{l}}} \cdots S_{i_{1}}$ with its $i, j$ th element $>0$. This allows the construction of an $M_{i}$ product of finite length such that all its entries are strictly positive. For each $i, j$, and each $S_{i}$ sequence that makes this entry positive, take the corresponding $M_{i}$ product. Then concatenate 
all such $M_{i}$ products-the existence of the $\mathbb{I}$ element in the $M_{i}$ ensures we effectively have a sum of matrix products, each with a different $i, j$ th entry assured of being $>0$. This, in addition to the fact the measure $\mu$ is discrete and finite, ensures the positivity condition is met. The result follows by Theorem 2 .

Now that we know that the distribution of values $\left\{\log \left\|M_{i_{m}} \cdots M_{i_{1}}\right\|_{e n}\right\}_{i_{m}, \ldots, i_{1}=0,1}$ converges weakly to a normal distribution, for both $\mu_{e}$ and $\mu_{p}$, it remains to calculate the mean and variance. As mentioned in the discussion at the start of Section 4, analytical methods for even the Lyapunov exponent tend to be few and far between, so numerical methods will be used.

The mean, $\mathbb{E}\left(\log \left\|S_{m}\right\|_{e n}\right)$ is simply the product of $m$, the sequence length, and the top Lyapunov exponent, the stable limit $\lambda$ from Theorem 1 . The variance is $m \sigma^{2}$, with

$$
\sigma^{2}=\lim _{m \rightarrow \infty} \frac{1}{m} \mathbb{E}\left(\log \left\|S_{m}\right\|-m \lambda\right)^{2} .
$$

We obtain not just the distribution of the logarithm of the norms, but also, the (normalized) norms themselves, simply by the composition of the random variable $\log \left(\left\|S_{m}\right\| / n\right)$ with the exponential function. We have a lognormal distribution for $\left\|S_{m}\right\| / n$

$$
\frac{\left\|S_{m}\right\|}{n} \sim \frac{1}{y} \frac{e^{-(\log y-m \lambda+\log n)^{2} /\left(2 m \sigma^{2}\right)}}{\sqrt{2 \pi m \sigma^{2}}} .
$$

It's important here to note several limitations. First, consider the theoretical expectation value of $\left\|S_{m}\right\| / n$ from (4.4), by the standard formula for lognormal expectation:

$$
\mathbb{E}\left(\frac{\left\|S_{m}\right\|}{n}\right)=\int y \frac{1}{y} \frac{e^{-(\log y-m \lambda+\log n)^{2} /\left(2 m \sigma^{2}\right)}}{\sqrt{2 \pi m \sigma^{2}}} d y=\frac{e^{m \lambda+m \sigma^{2} / 2}}{n} .
$$

Compared with the known expectation value of $\mathbb{E}\left(\left\|S_{m}\right\| / n\right)=2^{-m}$ from (4.3), we can see there is no way to reconcile these two values. In fact, the lognormal distribution estimate is quite accurate for the distribution of $\left\|S_{m}\right\| / n$ for $\log \left\|S_{m}\right\|$ within several standard deviations of $m \lambda$, but above this region (more positive), the estimates are increasingly poor as a function of $m$. In fact, they diverge at an exponential rate: the portion of the lognormal corresponding to $\log \left\|S_{m}\right\| / n>0$, the upper tail, has a weight that grows without bound in $m$; this in spite of the fact the random variable $\log \left\|S_{m}\right\| / n$ can never be greater than zero, by its definition as a probability distribution.

There are other limitations on the central limit theorem results. For one, the results only apply asymptotically as $m \rightarrow \infty$. And the theoretical mean, $m \lambda$, is only assured of being within $\mathcal{O}\left(\mathrm{m}^{1 / 2-\epsilon}\right)$ of the actual mean of the random variable $\log \left\|S_{m}\right\|$. And lastly, as $\delta$ approaches 0 , more and more of the weight of the probability distribution defined by the norms $\left\|S_{m}\right\|$ lies in fewer and fewer relatively large values. In fact consider that for fixed $p$, for any $m$ as $\delta \rightarrow 0$ we have the probability of each of the $n$ fully regular sequences approaching $1 / n$, while the mean of the distribution of the random variable $\log \left\|S_{m}\right\|$ drops as $m \lambda(\lambda<0)$. What happens then is the vast majority (nearly all $2^{m}$ ) of the norms become infinitesimal, following a normal distribution around $m \lambda$, while the vast majority of the probability mass lies in the longest surviving subsequences. As $\delta$ gets small, more 
and more of the probability mass will lie astronomically far in the upper tail of the central limit theorem-derived distributions. In fact, in Section 8 we will show that for all binary sequences under this method the asymptotic equipartition property (AEP) holds. The outliers that emerge in the upper tail as $\delta$ is reduced are the 'typical' sequences implied by the AEP. Large probability-mass outliers to the distributions on the norms can create some problems for numerical routines when the distributional entropy is computed, as will be seen in Section 8.

There are several numerical methods available for computing the Lyapunov exponent that give good rates of convergence. They usually try to take advantage of some feature of the random matrices in the support of $\mu$. These methods include, among others, weak-disorder expansion, microcanonical method, the replica trick, and cycle expansion $[13,36]$. The variance also may be computed with these methods, though it tends to require more samples for good convergence to a stable value, especially at low values of $\delta$. Because of this, the principal method used here is brute force Monte Carlo.

4.2. Brute force Monte Carlo. In practice, for the $M_{i}$ the most favorable numerical method for determining the Lyapunov exponent, $\lambda$, and the variance $\sigma^{2}$, for any value $p \in[1 / 2,1), p \in \mathbb{Q}$, and any $\delta \in[0,1]$, has been brute force Monte Carlo (BFMC). Cycle expansion was also tried $[9,35,36,41]$, but because cycle expansion showed untoward slowness in convergence, especially with the variance, out to order 23 whenever $\delta \approx 0$, its use was restricted to serving as a double check of the various Monte Carlo results.

Brute force Monte Carlo methods allow estimation of both the Lyapunov exponent and the variance, a.k.a. Lyapunov susceptibility [9]. A common routine is the following, based on selecting a random vector with non-negative entries, iterating it under randomly chosen $M_{i}$, while accumulating the magnification factor $(\alpha)$, which is a direct sampling of $e^{\lambda}$ (see for example [9] or [41]):

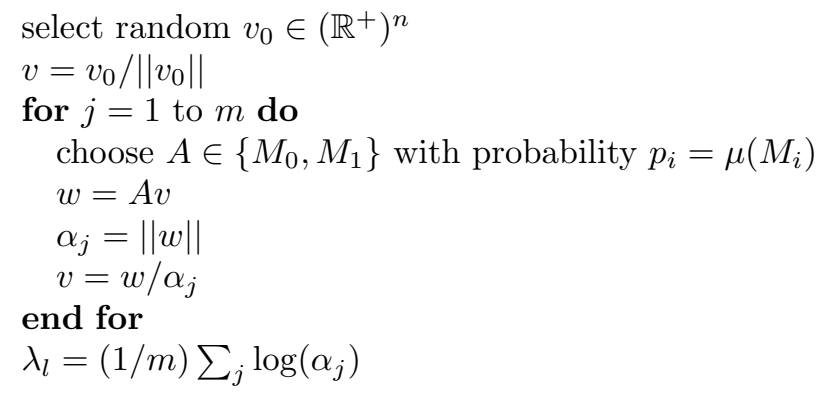

The algorithm is repeated for $j$ ranging over the number of desired samples. The $\lambda_{j}$ s are then averaged, to produce the final Lyapunov exponent estimate. The variance, $\sigma^{2}$, is estimated by $\left(\mathbb{E}\left(\lambda_{j}^{2}\right)-\left(\mathbb{E}\left(\lambda_{j}\right)\right)^{2}\right)$. Shown in Figure 5 are the Lyapunov exponents and variance plotted vs. $\delta \in[0,1]$ for the equiprobable $M_{i}$ weighting, $\mu_{e}$. The product length was $m=1 e 6$ and sample size was $N=1 e 5$, which are somewhat typical values [9]. For an accuracy estimate, we can treat the BFMC procedure as summing $N m$ i.i.d. normal random variables, each with variance $\sigma^{2}$. 

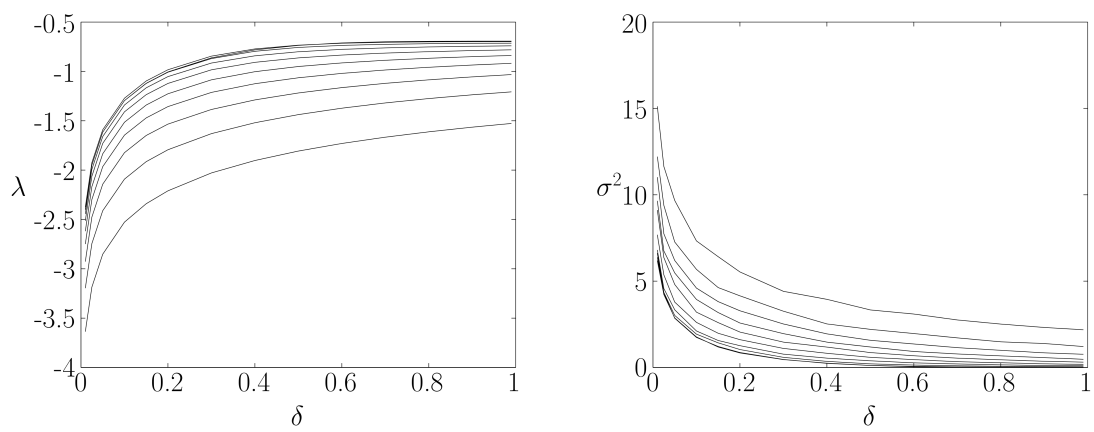

FiguRE 5. estimated Lyapunov exponents (left) and variances (right) plotted vs. $\delta$ for the evenly weighted $M_{i}$ distribution, $\mu_{e}$; $p$ values range from $1 / 2$ to $19 / 20$ at $1 / 20$ th intervals; the curves at any $\delta$ are monotonic in $p$ : the top curve on the $\lambda$ plot and the bottom curve on the $\sigma^{2}$ plot correspond to $p=1 / 2$; estimates were made using $B F M C$ at $m=1 e 6$, and $1 e 5$ samples; curve sets are asymptotic to $\mp \infty$ as $\delta \rightarrow 0$

By the law of large numbers, we expect the estimator for $\lambda$ to have error

$$
\mathcal{O}\left(\frac{\sigma}{\sqrt{N m}}\right)
$$

which for the chosen values of $N$ and $m$ is $\approx 1 e-5$.

We also have matrix distribution $\mu_{p}$, which weighs more heavily sequences with $p \approx\left(\# 0^{\prime} s\right) / m$. The advantage to this is that it gives us the distribution over sequences that are more likely to be of interest, while minimizing those with $\left(\# 0^{\prime} s\right) / m$ significantly different from $p$. In an extreme case, say $p=9 / 10$, almost all sequences in the distribution of norms derived with $\mu_{e}$ will have a far lower proportion of 0 's than $9 / 10$ ths, making it very likely that all sequences with approximately $m p=9$ zeros will be distribution outliers from the start. Using $\mu_{p}$ allows us to lens the distribution around the sequences with the proportion of zeros of interest. The results for $\mu_{p}$ are shown in Figure 6.

To check the parameters $\lambda$ and $\sigma^{2}$, we may sample a sufficiently large set of $M_{i}$ matrix product chains, with the $M_{i}$ chosen under the appropriate $\mu$, and plot a histogram of the logarithms of the entrywise matrix norms of the results. See Figure 7, which uses $\mu_{e}$.

4.3. Some example sequences; z-scores. The applicability of the central limit theorem now allows in addition to the probability of a given sequence, the calculation of a z-score, which allows the comparison of the regularity (high z-score) or irregularity (low z-score) between sequences of arbitrary types.

We note for the central limit theorem to apply, sample sequences must be of sufficient length. As a rough rule of thumb for adequate mixing to occur, experiments suggest sequences of length at least $10 n$, where $n$ is the matrix dimension, tend to give a reasonable approximation to a normal distribution. A sample frequency plot, or histogram of the $\left\|S_{m}\right\|$ values, as in Figure 7, can always be checked at the given 

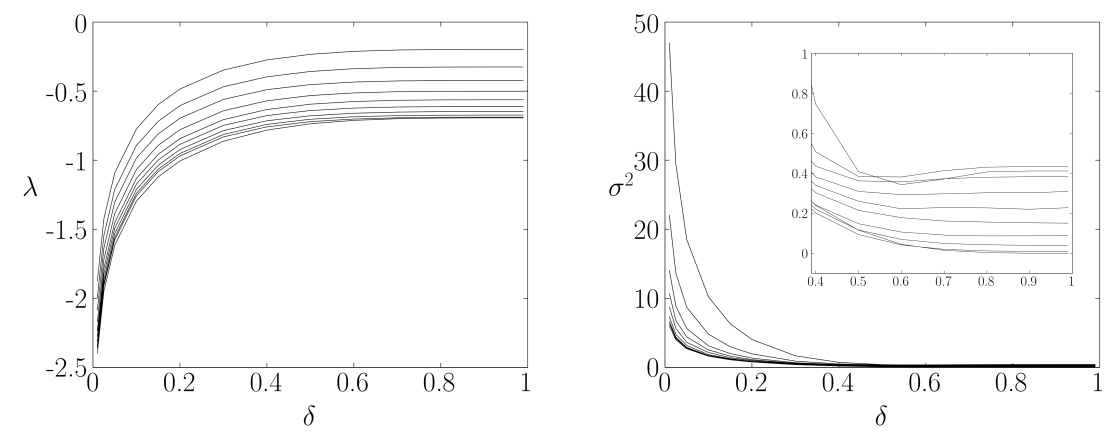

FiguRE 6. estimated Lyapunov exponents (left) and variances (right) plotted vs. $\delta$ for the p-proportional $M_{i}$ distribution, $\mu_{p}$; $p$ values range from $1 / 2$ to $19 / 20$ at $1 / 20$ th intervals; the curves, at least at $\delta \lesssim 0.4$, are monotonic in $p$ : the bottom curves in both plots correspond to $p=1 / 2$; estimates were made using BFMC at $m=1 e 6$, and $1 e 5$ samples; note curve sets are asymptotic to $\mp \infty$ as $\delta \rightarrow 0$

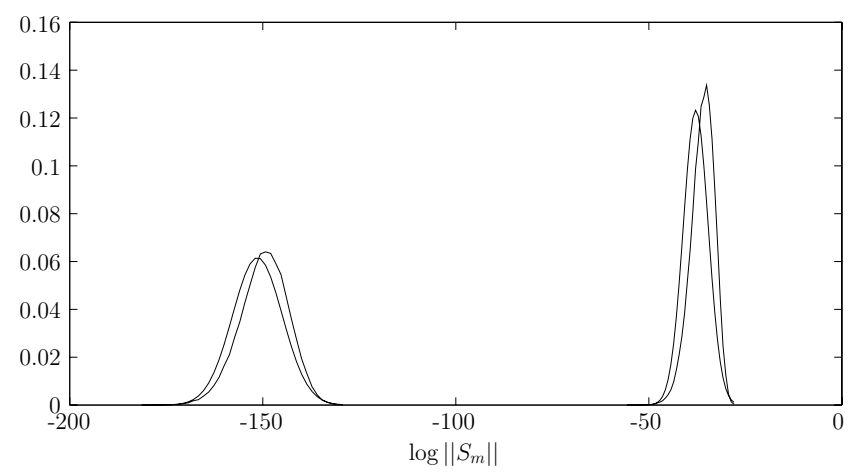

Figure 7. plots of theoretical vs. sampled frequencies of $\log \left\|S_{m}\right\|_{\text {en }}$ (1e5 samples, normalized) at $p=3 / 5$, and $m=50$ (rightmost bell curves) and $m=200$ (leftmost bell curves), under $\mu_{e}$; in both cases, the sampled curves are slightly right-shifted in relation to the theoretical Gaussians; the theoretical distribution curves were computed from the foregoing Monte Carlo trials, with estimates $\lambda \approx-0.75693$ and $\sigma^{2} \approx 0.2095$

sequence length, to verify the samples obey an approximate normal distribution, without for example undue skewness.

Figure 7 also indicates a further consideration for computing z-scores as accurately as possible for 'shorter' sequences, those say of length $\approx 10 n$.

Implicit in the Lyapunov exponent results of Theorem 1 is the permitted arbitrariness of the matrix norm. The accuracy of fit of any set of samples to the prevailing Gaussian under a given norm is then at best within some constant factor, since, at best, $\log \|A\|_{1} \in\left[\log \|A\|_{2}-c, \log \|A\|_{2}+c\right]$ under the equivalence of 
norms. In practice, an adjustment factor should be estimated when dealing with shorter product lengths. This is accomplished by deriving a sample estimate of the mean, $\mathbb{E}\left(\log \left\|S_{m}\right\|_{e n}\right)$, and calculating the correction factor as the difference between the sample mean and theoretical mean $m \lambda$. Experimentally, for several different $p$ values under the entrywise norm, the sample mean was found to typically lie to the right (less negative value) of the theoretical mean, as in Figure 7. The adjustment factor was also seen to be quite stable over values of $m$ in $[10 n, 50 n]$.

Assuming we are interested in z-scores for binary sequences with approximately $m p$ zeros, we use the estimates of $\lambda$ and $\sigma^{2}$ from the $\mu_{p}$ distribution over the matrix pair $M_{0}, M_{1}$. As discussed just above, the $\mu_{p}$ distribution ensures the distribution is focused on the sequences of interest.

Following are some examples at $p=4 / 5, \delta=0.1, m=50$, with estimates $\lambda=-1.0668, \sigma^{2}=2.5411$ (both estimated under $\mu_{p}$, using $P\left(M_{0}\right)=4 / 5, P\left(M_{1}\right)=$ $1 / 5)$, and adjustment factor, i.e. sample mean minus $m \lambda$, of 4.5 .

Note also that the central limit theorem and z-score calculation is applied to the variable $\log \left\|S_{m}\right\|_{e n}$, and not $\log \left(\left\|S_{m}\right\| / n\right)$-we do not normalize to fit to the Gaussian distributions.

$\begin{array}{ccc}\text { sequence s } & \log (\mathrm{P}(\mathrm{s})) & \text { z-score } \\ 00001000000000010000001001000010000001000001100000 & -30.204 & 1.7962 \\ 11000001010000100000001000001100100000000000010011 & -50.081 & 0.032734 \\ 01110111100000000000011100110000111011000100100101 & -78.712 & -2.5073\end{array}$

The first sequence is within a few splices of $\overline{10000}$ regularity. The middle sequence has a longer stretch of $0 \mathrm{~s}$, and several clusters of $1 \mathrm{~s}$, giving it a z-score near 0 . The last sequence is clearly more irregular, with long runs of clumped $1 \mathrm{~s}$, and also a long run of consecutive $0 \mathrm{~s}$, and an overall imbalance. Its over two and half standard deviations below the estimated mean of $m \lambda+\operatorname{adj}=-48.84$.

\section{Extension II: Pad-based methods}

The development up to this point has centered on taking an input sequence and, under the guise of splicing, looking for subregularity with respect to the $S_{i^{-}}$ generated template sequences; i.e. summing over subsequences of $S$ that happen to be fully regular, with an appropriate Bernoulli- $\delta$ weighting.

By analogy, the covered-covering relationship can be reversed. To complement the terms in Section 2.3, given a sequence $S$ of length $m$, and a fully regular sequence $F$ of length $\geq m$, we'll say that $S$ is superregular to $F$ if $F$ covers $S$ (recall, $F$ covers $S$ if $F$ contains $S$ as a subsequence). The superregular cover distance will be defined analogously to that of the subregular case, it being $|F|-|S| \geq 0$. Just as in the subregular case we could think of splicing bits from $S$ to form a fully regular subsequence, we'll define a pad as a bit added somewhere between (or on either end of) the bits of $S$, and will look for fully regular padded sequences containing $S$. It's worth noting that in parallel with Section 2.3, the superregular cover distance may be regarded as another simple string metric: another subcase of the Levenshtein distance, with only insertions allowed.

5.1. Padding method I: padding only. Recall the splicing dynamic, developed in Section 2.3. We may regard the splicing process, parametrized by $\delta$, as operating in the following way: we start with a matrix representation of the input sequence, 
coded in terms of $S_{i}$ 's, then proceed to check all subregular covers at distances $x \geq 0$. A splice consists of the removal of an $S_{i}$, the removal weighted by the respective Bernoulli probability value ( $p$ for the removal of a 0 bit, and $q$ for the removal of a 1 bit). We may consider that this scheme statistically favors an approximately $p$-proportion in its removal of 0 's, and a $q$ proportion in its removal of 1's. Further, the $\delta$ parameter allows smoothly weighting various sequences of a given subregular cover distance-all the way from fully favoring a subregular cover distance of 0 , when $\delta=0$, to fully favoring a subregular cover distance of $m$ (the length of the binary sequence; i.e. all bits have been spliced, and we're at the pure probability end) when $\delta=1$.

To develop a padding scheme by analogy, we again begin with the binary input sequence, $i_{1}, \ldots, i_{m}$, cast as a product of the respective $S_{i}$. We now however may pad (versus splice) a bit in anywhere we like, in the form of one of the $S_{i}$. And just as with splicing, where the removal of a bit was weighted by $p$ or $q$, under padding the inclusion of a bit will also incur a weight equal to its respective Bernoulli value. This achieves the desired analogous effect of statistically favoring an approximately $p$ proportion of padding 0 's, and a $q$ proportion of padding 1's. The $\delta$ parameter will again shift weight between elements of the original $S_{i}$ product, and the padded bits. To compare the two,

splice one bit $($ at $m-1):(1-\delta) S_{i_{m}}\left(\delta p_{i_{m-1}}\right)(1-\delta) S_{i_{m-2}} \cdots(1-\delta) S_{i_{1}}$ pad one bit (bet. $m$ and $m-1):(1-\delta) S_{i_{m}}\left(\delta p_{j} S_{j}\right)(1-\delta) S_{i_{m-1}} \cdots(1-\delta) S_{i_{1}}$, where $j$ is the padding symbol, and we've used $p_{0}=p, p_{1}=q$.

Just as splicing covered all subregular cover distances in $[0, m]$, padding should cover all superregular cover distances in $[0, \infty)$. We require a general product form analogous to the splice-based decomposition (2.3). To accomplish this, we construct padding matrix $P$ from the following geometric series (assuming it converges),

$$
P=\mathbb{I}+\delta\left(p S_{0}+q S_{1}\right)+\delta^{2}\left(p S_{0}+q S_{1}\right)^{2}+\ldots
$$

We then apply it to the original $S_{i}$ product as follows:

$$
P\left((1-\delta) S_{i_{m}}\right) P\left((1-\delta) S_{i_{m-1}}\right) P \cdots\left((1-\delta) S_{i_{1}}\right) P .
$$

This has the desired effect of forming a sum over every possible combination of padding matrices, $\delta p_{i} S_{i}$, inserted between the bits corresponding to the binary $i_{1}, \ldots, i_{m}$ sequence under test.

To make the distribution of entrywise norms over all possible input sequences a probability distribution, we need the normalization constant at a given $m$. This is simply,

$$
c_{N}=\frac{1}{\left\|P\left((1-\delta)\left(S_{0}+S_{1}\right)\right) P \cdots\left((1-\delta)\left(S_{0}+S_{1}\right)\right) P\right\|_{e n}} .
$$

To form the probability values from $(5.2)$, we notice the $(1-\delta)$ values may be factored out, producing,

$$
P\left(i_{1}, \ldots, i_{m}\right)=c_{N}\left\|P S_{i_{m}} P S_{i_{m-1}} \cdots S_{i_{1}} P\right\|_{e n},
$$

where the normalization constant has simplified to,

$$
c_{N}=\frac{1}{\left\|P\left(\left(S_{0}+S_{1}\right) P\right)^{m}\right\|_{e n}} .
$$

Just as in the splicing case, where at $\delta=0$ we had the pure $S_{i}$ product, and at $\delta=1$ we had only a product of the splicing terms, $\delta p_{i} \mathbb{I}$, in (5.3), at $\delta=0$ we 
have again the original $S_{i}$ product, and at $\delta=1$ we have only products of the padding terms, $\delta p_{i} S_{i}$. In fact, when $\delta=1$, all superregular cover distances receive equal weighting (and with Bernoulli coefficients)-for the series to have a chance to converge this is the best we can do (we can't indefinitely shift weight toward longer and longer padding arrangements and still have the series converge).

It remains to show convergence of the geometric series of (5.1). From the (double) stochasticity of $S_{0}+S_{1}$, the fact all entries are non-negative, and that $q \leq p<1$, we have,

$$
\left\|\left(\delta\left(p S_{0}+q S_{1}\right)\right)^{k}\right\|_{e n} \leq(\delta p)^{k}\left\|\left(S_{0}+S_{1}\right)^{k}\right\|_{e n}=n(\delta p)^{k} .
$$

Then we invoke the equivalence of norms (i.e. the series in $P$ converges under any norm), proving convergence. Since $\mathbb{I}-\delta\left(p S_{0}+q S_{1}\right)$ is non-singular and therefore invertible, we can write,

$$
P=\frac{1}{\mathbb{I}-\delta\left(p S_{0}+q S_{1}\right)} .
$$

5.2. Padding method II: padding and splicing. Padding and splicing may also be combined. This allows evaluating a test sequence for its closeness to the template sequences under the joint operations of both padding and splicing. We'll need a matrix representation that allows both splicing from the input sequence, and padding to it (and preferably avoiding the redundancy of splicing a pad, or vice-versa).

This is achieved by simply modifying the input sequence's representation from its terms in $S_{i}$ in the pad-only case, to using the $M_{i}$ of (3.1). The padding matrices, $P$, remain unchanged. A simple example of 01 is represented as,

$$
P M_{0} P M_{1} P \text {, }
$$

which will permit padding the input sequence, like $0_{P} 0_{I} 1_{I} 1_{P}$, splicing from it, e.g. $1_{I}$, and trying both, $0_{P} 1_{I} 1_{P}$, etc., (where the $I$ - and $P$-subscripted bits refer to the Bernoulli- $\delta$ weighted $S_{i}$ from the input sequence and pad portions respectively). Increasing the $\delta$ parameter from 0 will simultaneously emphasize longer and longer subregular cover distances for the input sequence, and longer and longer superregular cover distances for the padding portions.

In general, the $\delta$ weighting for $\delta<1$ will penalize in proportion to the number of pads and/or splices required to achieve full regularity of the input sequence. The optimal number of such operations under the combined padding and splicing is most closely related to the Levenshtein distance. While the Levenshtein distance counts insertions, deletions, and substitutions equally, here a substitution is formed by a pad and a splice, and so gets a count of 2 . We would expect the minimum Levenshtein distance, taken over all pairs $\left\{S, T_{j}\right\}_{j}$, where $T_{j}$ is a template sequence of length $j$, to serve as a first order approximation of the relative magnitude of $\left\|P M_{i_{m}} \cdots M_{i_{1}} P\right\|$. Such a distance approximates the minimal number of pads and/or splices required to make the test sequence fully regular.

The probability distribution is formed analogously to the pad-only scheme just above: given a binary input sequence $\left(i_{j}\right)_{j=1 \ldots m}$, compute

$$
\left\|P M_{i_{m}} P \cdots P M_{i_{1}} P\right\|_{e n}
$$

then normalize with

$$
c_{N}=\frac{1}{\left\|P\left(\left(M_{0}+M_{1}\right) P\right)^{m}\right\|_{e n}} .
$$


5.3. Central limit theorem for padding. Assuming $\delta>0$, the central limit theorem also applies to the two padding approaches just developed. To show this, recall the padded products have form

$$
P V_{i} P \cdots V_{i} P
$$

where we've abused the notation slightly: each of the $V_{i}$ stands for some $S_{j}$, or $M_{j}$, $j \in[1, k]$, for pad-only and pad-and-splice respectively, and,

$$
P=\sum_{l=0}^{\infty}\left(\delta\left(p S_{0}+q S_{1}\right)\right)^{l} .
$$

We'll rewrite the product in (5.6) as,

$$
P V_{i} P \cdots V_{i} P=\sqrt{P}\left(\sqrt{P} V_{i} \sqrt{P}\right) \cdots\left(\sqrt{P} V_{i} \sqrt{P}\right) \sqrt{P} .
$$

We then note the entries of matrix $\sqrt{P}$ are strictly positive. This follows from the binomial expansion,

$$
\sqrt{P}=\frac{1}{\sqrt{\mathbb{I}-\delta\left(p S_{0}+q S_{1}\right)}}=\mathbb{I}+\frac{1}{2} \delta\left(p S_{0}+q S_{1}\right)+\frac{3}{8}\left(\delta\left(p S_{0}+q S_{1}\right)\right)^{2}+\ldots,
$$

and the fact $S_{0}+S_{1}$ is irreducible. Next note that all elements of $\sqrt{P} V_{i} \sqrt{P}$ are strictly positive. This follows from the fact the $V_{i}$ are non-negative, and have at least one strictly positive element. The random product pair, $\left\{\sqrt{P} V_{0} \sqrt{P}, \sqrt{P} V_{1} \sqrt{P}\right\}$, as the support of any Bernoulli probability measure, $\mu$, then trivially meets the conditions of Theorem 2.

It remains to address the leading and trailing $\sqrt{P}$ matrices in the product in (5.7). Since all elements of $\sqrt{P}$ are bounded away from 0 and $\infty$, the effects of left or right multiplication by $\sqrt{P}$ on the entrywise norm of products of $\sqrt{P} V_{i} \sqrt{P}$ is no more than a constant (bounded) factor, which is absorbed in the central limit theorem on the log of the norm.

To estimate the Lyapunov exponents and variances of the padding distributions, we apply BFMC to the matrix pair $\left\{V_{0} P, V_{1} P\right\}$. (When computing a z-score, the same pair may be used.) Again, the effect of leaving off the leading padding matrix, $P$, has the effect of altering the norm by a constant factor, amounting to a constant offset to the $\log$ of the norm. This becomes negligible in the limit $m \rightarrow \infty$. It may also be mitigated by the computation of an adjustment factor, as in Section 4.3.

See Figure 8 for plots of the Lyapunov exponents and variances as a function of $\delta$ for the pad-only distributions, and Figure 9 for plots of the Lyapunov exponents and variances for the pad-and-splice distributions. Both were computed with brute force Monte Carlo at $m=2 e 5$ and $5 e 4$ samples (shorter sample runs showed good stability for both the padding cases, over the splicing case's $m=1 e 6$ and $1 e 5$ samples, probably owing to the greater element-wise positivity of the padding sequence's $V_{i} Q$ components). Compare with the splice-only plots of Figure 6 .

5.4. Examples for all three methods: splice-only, pad-and-splice, and pad-only. Some example sequences will be helpful to show how the pad-only, and pad-and-splice distributions rank binary sequences. As with the example in Section 4.3 , we'll use $p=4 / 5, \delta=0.1$. For any given binary sequence, we compute the matrix product,

$$
Q V_{i_{m}} Q V_{i_{m-1}} \cdots Q V_{i_{2}} Q V_{i_{1}} Q
$$



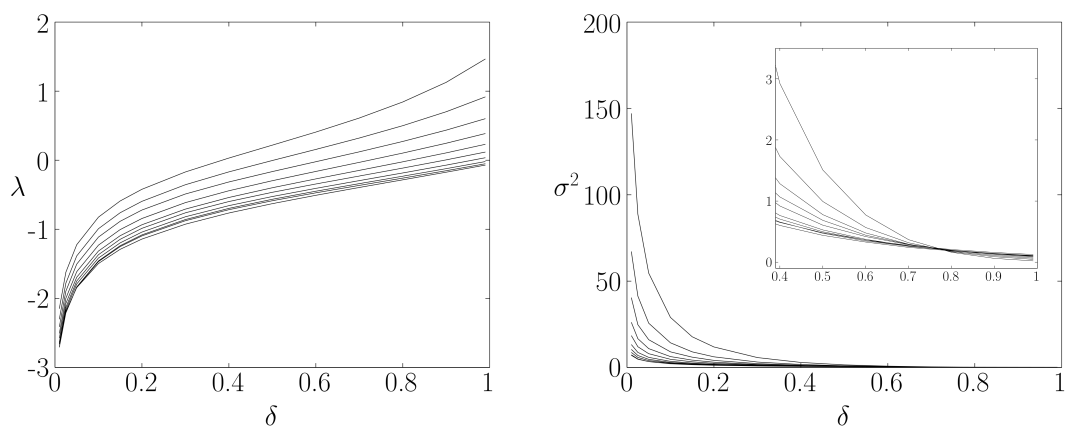

FiguRE 8. estimated Lyapunov exponents (left) and variances (right) plotted vs. $\delta$ for the pad-only distribution under the $p$ proportional $\mu_{p}$ weighting of the $M_{i}$ matrices; $p$ values range from $1 / 2$ to $19 / 20$ at $1 / 20$ th intervals; at any $\delta \lesssim 0.7$, the curves are monotone in $p$; in both plots, the lowermost curves, in at least the $\delta \lesssim 0.7$ region, correspond to $p=1 / 2$; estimates were made using BFMC at $m=2 e 5$, and $5 e 4$ samples; note curve sets are asymptotic to $\mp \infty$ as $\delta \rightarrow 0$
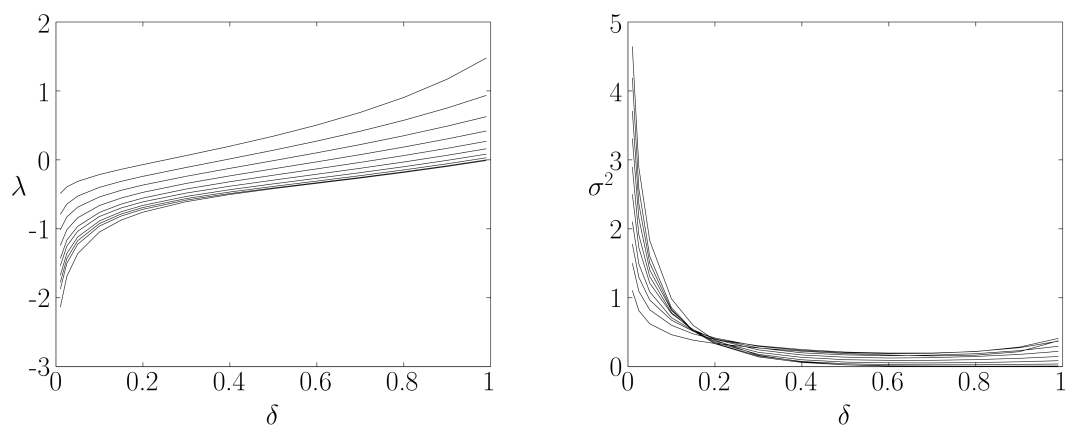

FiguRE 9. estimated Lyapunov exponents (left) and variances (right) plotted vs. $\delta$ for the pad-and-splice distribution under the $p$ proportional $\mu_{p}$ weighting of the $M_{i}$ matrices; $p$ values range from $1 / 2$ to $19 / 20$ at $1 / 20$ th intervals; $\lambda$ curves at any $\delta$, and the $\sigma^{2}$ curves at a given $\delta \lesssim 0.15$, are monotone in $p$; the curve corresponding to $p=1 / 2$ is lowermost in the $\lambda$ plot, and uppermost in the $\sigma^{2}$ plot (left end); estimates were made using BFMC at $m=2 e 5$, and $5 e 4$ samples; note curve sets are asymptotic to $\mp \infty$ as $\delta \rightarrow 0$

where for splice-only, the padding $Q$ is simply $\mathbb{I}$, and $V_{i}$ is the appropriate $M_{i}$ $(i \in\{0,1\})$ from the end of Section 3; for pad-only, the padding $Q$ is the $P$ matrix of Section 5.1, and $V_{i}$ is the corresponding $S_{i}$; for pad-and-splice, the padding $Q$ is the same $P$ matrix, while the $V_{i}$ is the original $M_{i}$ from the splice-only case. The entrywise norm of the resulting matrix product is then gauged against the central limit theorem's distribution, allowing the computation of z-scores. To help 
understand how the three methods weigh binary sequences, a reformulation of the splice-only $\delta$ polynomial, (2.3), in broader terms of pads and splices will be useful.

Recall given sequence $S$, for splice-only we denoted by $\left(X_{i}\right)_{Q(k)}$ the product of $X_{i}$ corresponding to $k$-bit subsequence $Q(k)$. The $(m-k)$-bit complement of subsequence $Q(k)$ in $S$ was denoted by $Q^{c}(k)$. To include the padding schemes, we'll denote by $R(k)$ a $k$-bit sequence of pads (e.g. if 01 is padded to 1011 , then $R(2)=11)$. Including the prime $(')$ notation, we'll denote by $\left(S_{i}\right)_{Q^{\prime}\left(m-l_{s}\right) \cup R^{\prime}\left(l_{p}\right)}$ a surviving sequence of $S_{i}$ generated by some set of $l_{s}$ splices $\left(l_{s} \in\{0, \ldots, m\}\right)$ and $l_{p}$ pads $\left(l_{p} \in\{0,1, \ldots\}\right)$ applied to $S$. We then have the following general decomposition of the matrix product of $(5.8)$, ordered in terms of the total number of padding and splicing operations used $\left(l_{s}+l_{p}\right)$ :

$$
\begin{aligned}
& (1-\delta)^{m}\left(S_{i}\right)_{Q^{\prime}(m)}+ \\
& \quad \sum_{j: l_{s}+l_{p}=1} \delta^{l_{s}+l_{p}}(1-\delta)^{m-l_{s}}\left(r_{i}\right)_{Q_{j}^{\prime c}\left(m-l_{s}\right)}\left(r_{i}\right)_{R_{j}^{\prime}\left(l_{p}\right)}\left(S_{i}\right)_{Q_{j}^{\prime}\left(m-l_{s}\right) \cup R_{j}^{\prime}\left(l_{p}\right)}+ \\
& \quad \sum_{j: l_{s}+l_{p}=2} \delta^{l_{s}+l_{p}}(1-\delta)^{m-l_{s}}\left(r_{i}\right)_{Q_{j}^{\prime c}\left(m-l_{s}\right)}\left(r_{i}\right)_{R_{j}^{\prime}\left(l_{p}\right)}\left(S_{i}\right)_{Q_{j}^{\prime}\left(m-l_{s}\right) \cup R_{j}^{\prime}\left(l_{p}\right)}+\ldots
\end{aligned}
$$

The two product $\left(r_{i}\right)$ terms are the combined product of Bernoulli factors, $p$ 's and $q$ 's, resulting from the associated pads and splices used. Combined with the leading $\delta$ terms, they constitute the weighting penalty. The $\left(S_{i}\right)$ term represents a surviving $S_{i}$ product.

Consider the examples of Table 1 , under $p=4 / 5, \delta=0.1$. To compute the zscores, the procedure of Section 4.3 is followed: computing the central limit theorem parameters for $\mu_{p}$, the $p$-proportionally weighted random matrix distribution (as we'll be mainly interested in sequences with approximately $m p$ 0s), with support $S_{i} P$ for pad-only, and support $M_{i} P$ for pad-and-splice; ensuring the sequence length is adequate for the central limit theorem to apply $(\gtrsim 10 n)$; and the estimation of an adjustment factor, to approximate the difference between the theoretical mean, $m \lambda$, and the sample mean.

All sequences in Table 1 are of length $m=60$. For pad-only, the values used under $\mu_{p}$ were $\lambda=-1.2292, \sigma^{2}=6.3077$, and an adjustment factor of 3.0. For pad-and-splice under $\mu_{p}, \lambda=-0.66281, \sigma^{2}=0.70959$, and 2.0 was used for the adjustment factor. Also for comparison are splice-only probabilities and z-scores from Section 4.3: $\lambda=-1.06681, \sigma^{2}=2.5411$, with adjustment factor 4.5 . For the notation, $\mathrm{P}_{s}$ denotes splice-only; $\mathrm{P}_{p, s}$ denotes pad-and-splice; and $\mathrm{P}_{p}$ denotes pad-only distributions.

Examining the z-scores for $s_{1}$, they are highest for the splice-only distribution. This reflects the existence of several clustered 1s, notably 11 and 101, which are easily removed via splicing, but would require many more pads to make regular on $p=4 / 5$.

The sequence $s_{2}$ is favored by the pad-only distribution. The minimal number of pads required to make $s_{2}$ fully regular is 16 , which produces the corresponding moderately high z-score. Contrast this with pad-and-splice, which comes in at almost a full standard deviation lower. To explain the disparity, consider that with pad-only there are 15 possible padding levels that will produce, roughly, sequences with higher z-scores (e.g. a sequence that required only 15 pads to become fully 
TABLE 1

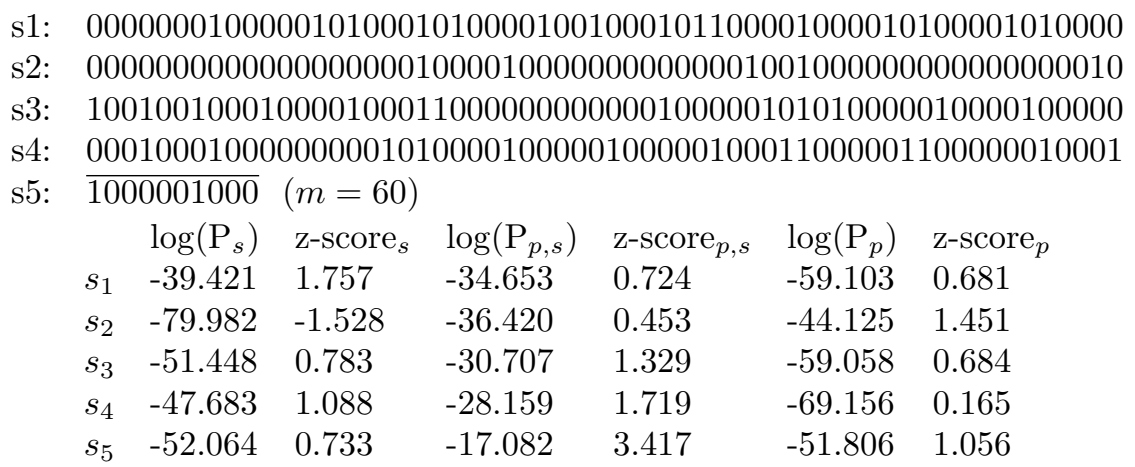

regular would, on average, have a higher z-score than $s_{2}$ ). In contrast, for padand-splice, there are $15(16) / 2=120 \mathrm{pad}$ and/or splice levels that would produce sequences with higher z-scores: i.e. if a minimum of 16 total operations (pads) are required to make the sequence regular, then any sequence that required 15 or fewer operations to make it regular will likely have a higher z-score. At 15 operations we are allowed 15 pads, or 14 pads and 1 splice, etc. for a total of 15 . The total number of sequence groups with higher z-scores then is $15+\ldots+1=120$. Consider further that a sequence with $p=4 / 5, m=60$ has, on average, around $121 \mathrm{~s}$. With a combination of 24 pads and splices, we could render almost all sequences at this $p$ value fully regular. Considering it's likely some of the 1s don't need relocating, it's not unreasonable that $\approx 16 \mathrm{pad}$ and/or splice operations would make a considerable proportion of sequences regular. In addition, a sequence such as $s_{2}$ is not amenable to splicing, which makes the use of pad-and-splice less efficient; with mostly only pads at its disposal, the multiplicity of surviving sequences at a given $l_{p}+l_{s}$ level is reduced, further lowering the z-score (consult (5.9)).

The sequence $s_{3}$ is moderately favored by pad-and-splice. This is seen by noting the long central run of $0 \mathrm{~s}$, which favors padding, and the clumped 1s, which favor splicing. In sequence $s_{4}$ the balance is tipped toward splicing, because, in spite of the early run of nine $0 \mathrm{~s}$, the sequence is fairly easy to make regular with splices. Pad-and-splice again shows the highest z-score.

Sequence $s_{5}$ strongly favors pad-and-splice. Simply shifting $1 \mathrm{~s}$, a total of 12 operations, 6 pads and 6 splices, will make it fully regular. In addition, a 0 may be spliced to the left of the odd-numbered $1 \mathrm{~s}$ in the sequence (with 5 ways to do so), and a 0 padded to the right of the same 1s (4 choices for pad positions) to make it fully regular. Because of the near-regularity of the sequence as it is, the multiplicity of these and other higher order pad-and-splice string edits is quite high. Such sequences, along with more on the differences and benefits of each of the three main methods, will be looked at again in Section 9.

\section{Extension III: increasing the proportion spread; matrix dimensions

$$
>d_{0}+d_{1}
$$

It is possible to loosen the conditions on what constitutes a fully regular sequence. Consider the effect of expanding the matrix dimension, call it $s$, while leaving the other parameters unchanged. We have, 
(1) $s \geq n=d_{0}+d_{1}$

(2) $\operatorname{gcd}\left(d_{0}, d_{1}\right)=1$

(3) $d_{0} \leq d_{1}$

where $p$ remains defined as $p=d_{1} /\left(d_{0}+d_{1}\right)$. As we'll see, allowing matrix dimension $s$ to be as large as we'd like provides a wider range of admissible fully regular sequences. It broadens the allowed local proportion of 0 s on contiguous blocks of a given length, while maintaining the proportion $p$ in the $\operatorname{limit} \lim _{m \rightarrow \infty}(\# 0 s) / m=$ $p$.

As an example, consider $d_{0}=d_{1}=1$, and $s=6$. This is similar to the basic $p=1 / 2$ case above (indeed, $p$ remains the same), but now with $6 \mathrm{x} 6$ matrices:

$$
M_{0}=\left[\begin{array}{cccccc}
p \delta & 1-\delta & 0 & 0 & 0 & 0 \\
0 & p \delta & 1-\delta & 0 & 0 & 0 \\
0 & 0 & p \delta & 1-\delta & 0 & 0 \\
0 & 0 & 0 & p \delta & 1-\delta & 0 \\
0 & 0 & 0 & 0 & p \delta & 1-\delta \\
0 & 0 & 0 & 0 & 0 & p \delta
\end{array}\right], M_{1}=\left[\begin{array}{cccccc}
q \delta & 0 & 0 & 0 & 0 & 0 \\
1-\delta & q \delta & 0 & 0 & 0 & 0 \\
0 & 1-\delta & q \delta & 0 & 0 & 0 \\
0 & 0 & 1-\delta & q \delta & 0 & 0 \\
0 & 0 & 0 & 1-\delta & q \delta & 0 \\
0 & 0 & 0 & 0 & 1-\delta & q \delta
\end{array}\right]
$$

Admissible fully regular sequences are analogous to random walks of steps right or steps left of size 1 on a segment with six total positions. This allows fully regular sequences such as $1111100000 \ldots$, which were not admissible when the matrix dimension was 2 . The proportion spread, or degree of local variation permitted on any substring, has increased. As a consequence, when $s>d_{0}+d_{1}$ the number of sequences considered fully regular grows in geometric proportion to the length of the sequence.

The geometric growth may be shown by recalling that the quantity $\|\left(S_{0}+\right.$ $\left.S_{1}\right)^{m} \|_{\text {en }}$ counts all walks of width 2 (which form one set of unique binary sequences), weighted by their positional multiplicity on the line segment of length $s-1$ (so weight of $s-1$ ); all walks of width 3 (another set of unique binary sequences), weighted by their multiplicity $(s-2)$; and so on. We note that $S_{0}+S_{1}$ is non-negative and irreducible, and that when $s>d_{0}+d_{1}$, the max row (or column) sum is 2 and the min row (or column) sum is 1 . This allows application of PerronFrobenius: for a non-negative irreducible matrix with non-identical row sums, the top eigenvalue lies between the min and max row sum, the inequalities strict. In this case, the top eigenvalue obeys $1<\lambda<2$. This means,

$$
s \text { (\# of unique fully regular binary sequences) } \gtrsim \lambda^{m},
$$

where $\lambda>1$.

For the splice-only probability distribution in the more general $s>d_{0}+d_{1}$ case, we no longer have stochasticity of the matrix sum $M_{0}+M_{1}$ (unless $\delta=1$ ). So the normalization constant is no longer $n$, but must be computed as,

$$
c_{N}=\frac{1}{\left\|\left(M_{0}+M_{1}\right)^{m}\right\|_{e n}} .
$$

The normalization constants for pad-only and pad-and-splice remain the same as in equations (5.4) and (5.5), respectively.

We also need to ensure in this more general $s>n$ case the geometric series encoded in the padding terms, $P$, of (5.1), converges. This follows from the following claim:

Claim 4. Given $d_{0}+d_{1} \leq s$ with $\operatorname{gcd}\left(d_{0}, d_{1}\right)=1$, there exists some $\epsilon>0$ and fixed $c$ such that for all $\delta \in[0,1]$ and all $k \geq 0$,

$$
\left\|\left(\delta\left(p S_{0}+q S_{1}\right)\right)^{k}\right\|_{e n}<c(1-\epsilon)^{k} .
$$


Proof. The claim is trivial when $\delta=0$, so assume $\delta>0$. In aim of applying Perron-Frobenius, we need to show irreducibility of $S_{0}+S_{1}$ in the general case, $s \geq d_{0}+d_{1}$.

Decompose $[1, s]$ into disjoint segments $\left\{l_{k}\right\}_{k=0, \ldots, k_{\max }-1}$, where $k_{\max }=\left\lfloor s /\left(d_{0}+\right.\right.$ $\left.\left.d_{1}\right)\right\rfloor$, with $l_{k}=\left[1+k\left(d_{0}+d_{1}\right),(k+1)\left(d_{0}+d_{1}\right)\right]$, and adding, if necessary, a remainder segment $l^{\prime}=\left[1+k_{\max }\left(d_{0}+d_{1}\right), s\right]$. We have $[1, s]=\cup_{k=0}^{k_{\max }-1} l_{k} \cup l^{\prime}$.

A covering walk is done by linking neighboring Christoffel words. Starting in any $l_{i}$, the walk associated with the $n=d_{0}+d_{1}$-long Christoffel word is performed. This covers all spots in $l_{i}$. We then take steps of size $d_{0}$ (or $d_{1}$ ) to arrive in $l_{i-1}$ (resp. $l_{i+1}$ ), and repeat the Christoffel word formation walk in this segment. The process is repeated until all segments $l_{j}$ are completely covered, as well as the remainder segment $l^{\prime}$, if applicable.

This proves $S_{0}+S_{1}$ is an irreducible matrix, and that for arbitrary entry $i, j$, there exists some $k$ th power such that $\left(\left(S_{0}+S_{1}\right)^{k}\right)_{i, j}=1$. The irreducibility of $\delta\left(p S_{0}+q S_{1}\right)$ when $\delta>0$ follows immediately. We can then apply Perron-Frobenius as just above, noting that the max row sum of $\delta\left(p S_{0}+q S_{1}\right)$ is exactly $\delta$, and the min row sum is $\delta q, q \leq 1 / 2$. Taking $\delta=1$ as the worst case, we have the top eigenvalue, $\lambda$, obeying $\lambda<1$ for all $\delta \in[0,1]$. This proves the claim.

The central limit theorem for the pad-and-splice case, and the pad-only case follows from the same strict entrywise positivity proof just above in Section 5.3, taking into account the irreducibility proof for $S_{0}+S_{1}$ in Claim 4 , which shows $(\sqrt{P})_{i, j}>0$. For the splice-only case, we have established the irreducibility of the set $\left\{S_{i}\right\}_{i}$, again by Claim 4 . That this case obeys the central limit theorem follows from the same reasoning as in the proof of Corollary 1, in Section 4.1.

Shown in Figure 10 are the results of brute force Monte Carlo estimations on the Lyapunov exponents and variances in the case of $d_{0}=1, d_{1}=4(p=4 / 5)$ for pad-and-splice, while $s$ is varied: $s \in\{5,7,9,11,13,15\}$.

\section{Extension IV: alphabets with more than 2 symbols}

Consider an alphabet $\mathcal{A}$ with more than two symbols, e.g. $\{a, b, c\}$, and an associated discrete random variable under some generalized Bernoulli distribution. One approach would be to simply apply the preceding binary regularity scheme one letter at a time, e.g. treating a.a..aa..aa.. as 1010011001100, computing its regularity score, then repeating for $b$ and $c$. We might finally aggregate the letter scores via, for example, an arithmetic average. A drawback with this approach is its failure to adequately credit subsequences that might fit a fully regular template. For example, in the equiprobable case, sequences of type $\overline{a b c}$ and its cyclic rotations would be considered fully regular. In this case, though the by-letter regularity scores of baaaaccc and aaaabccc might be the same, the second sequence contains a consecutive $a b c$, and we'd like the measurement scheme to reflect this.

A more comprehensive approach can be developed by extending the binary alphabet's $S_{i}$ matrices to matrices whose product vanishes for all but those associated with some set of 'fully regular' sequences on the larger symbol set.

7.1. Simplices introduction. Recall the effects of the binary shift matrices, $S_{0}$ and $S_{1}$, on the column vector $v$ (Section 3.1). Viewing $v$ as a line segment, the $S_{i}$ effectively shifted the segment up or down, with cutoffs at each end. Each shift 

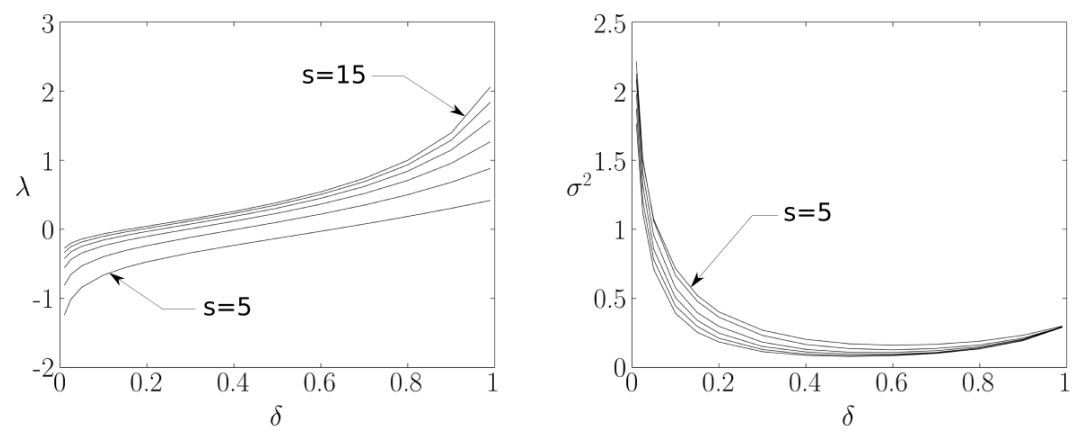

FiguRE 10. estimated Lyapunov exponents (left) and variances (right) plotted vs. $\delta$ for the pad-and-splice distribution under the p-proportional $\mu_{p}$ weighting of the $M_{i}$ matrices, while $s$ is allowed to vary; $p$ is fixed at $4 / 5\left(d_{0}=1 ; d_{1}=4\right), s=5,7, \ldots, 15$; at all $\delta$ for the $\lambda$ curves, and for $\delta$ approximately in $[0.1,0.7]$ for the $\sigma^{2}$ curves, the curves are monotone in $p$; the lowermost curve in the $\lambda$ plot, and the uppermost curve for most of the $\sigma^{2}$ plot correspond to $s=5 ; B F M C$ at $m=2 e 5$, and $5 e 4$ samples; note curve sets are asymptotic to $\mp \infty$ as $\delta \rightarrow 0$

direction corresponded to a different symbol, in particular 0 or 1 . It is natural to consider extending this approach to alphabets of more than two symbols, perhaps using some analog of simple line segments in higher dimensions. For three symbols, for example, we would require three different directions. We'd also like a shift in any one direction to affect the other two; this precludes orthogonal directions, which would cause one symbol's frequency to be decoupled from the others'. These conditions may be met with regular simplices as follows.

Consider alphabet $\mathcal{A}=\left\{\alpha_{1}, \ldots, \alpha_{k}\right\}$, and a corresponding set of generalized Bernoulli probabilities, $p_{i}>0, p_{1}+\ldots+p_{k}=1$.

Consider the boundary simplex, $B$, consisting of the faces of a regular $k-1$ dimensional simplex of height $h$. Coincident to the boundary, fit the $(k-1)-$ simplex $X_{0}$ (also of height $h$ ). To each of the $k$ faces of $X_{0}$ assign a distinct symbol from $\mathcal{A}$. Let $\mathbf{n}_{i}$ be the inward-pointing unit normal to face $\alpha_{i}$. Denote shift $c \Psi_{i}$ as a shift of $X_{0}$ in the direction $\mathbf{n}_{i}$ by amount $c$. In parallel with the binary symbol case, where the shifted line segment lost all of what extended beyond either of the two end cutoffs, we consider whatever of $X_{0}$ has extended beyond the boundary simplex $B$ after the first shift to be removed, or sheared off.

Let $\left(X_{0}, X_{1}, \ldots\right)$ denote an orbit of $X_{0}$ under some series of shift operations $c_{1} \Psi_{i_{1}}, c_{2} \Psi_{i_{2}}, \ldots$

Discretize $h: h=u /(k-1), u \geq 1, u \in \mathbb{N}$. Each of the $k$ altitudes of $X_{0}$ will be composed of $h(k-1)$ equal segments of length $1 /(k-1)$. Note that the hyperfaces of a regular $k-1$-simplex meet at an angle (the dihedral angle) of $\cos ^{-1}(1 /(k-1))$. If we then consider a shift of amount $l, l \in \mathbb{N}$, made in direction $\mathbf{n}_{i}$, then face $\alpha_{i}$ has moved by the amount $l$ in the same direction, and faces $\alpha_{j}, j \neq i$, are shifted in respective directions $-\mathbf{n}_{i}$ by the amount $l /(k-1)$. Given the shearing at the boundary, this amounts to all faces but $\alpha_{i}$ losing a layer of thickness $l /(k-1)$. 
Note that removing a uniform layer of thickness $t$ from the hyperface of any regular simplex of altitude $h$ leaves us with a regular simplex of altitude $h-t$.

An orbit of $X_{0}$ will then amount to the various shearing of faces, always by an amount in integer multiples of $1 /(k-1)$. Define a unit shear cell as the smallest possible simplex left after some set of shearing operations. Unit shear cells will naturally have all faces parallel to the faces of $B$, and will have height $1 /(k-1)$.

Figure 11 compares the shift of a five-unit line segment by two units with the $l=1$ shift of a 2 -simplex of altitude 2 , with the subdivisions in units of $1 /(k-1)=$ $1 / 2$. The outer dashed lines indicate the boundary $B$; the thickened outer lines indicate $X$; and the shaded interior simplices indicate the unit shear cells.
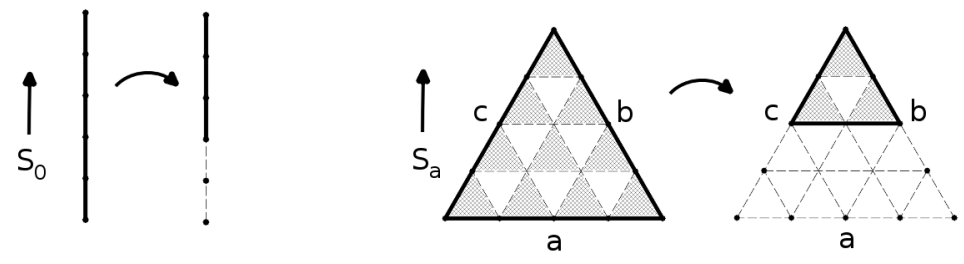

FigURE 11. shifting a 1-d simplex and shifting a 2-d simplex; shaded areas indicate unit shear cells

Analogous to the binary case, for a particular set of symbol probabilities we assign to each shift direction $\mathbf{n}_{i}, i=1, \ldots, k$, a whole number distance $d_{i}$. We'd like to translate the shift-distance set $\left\{d_{1}, \ldots, d_{k}\right\}, \operatorname{gcd}\left(d_{1}, \ldots, d_{k}\right)=1$, into the generalized Bernoulli probabilities corresponding to each symbol. A 'full orbit' of the simplex can be constructed by finding $r_{l}=\operatorname{lcm}\left\{d_{1}, \ldots, d_{k}\right\}$, and defining a 'step' corresponding to face $\alpha_{i}$ as the corresponding shift of the simplex in amount $d_{i}$ in direction $\mathbf{n}_{i}$. We then take $r_{l} / d_{1}$ steps for $\alpha_{1}$, then $r_{l} / d_{2}$ steps for $\alpha_{2}$, and so on, noting by construction the distance gone at each set of steps in direction $\mathbf{n}_{i}$ is the same. This forms a closed path, returning the simplex to its starting point. Taking the proportion of each $\alpha_{i}$ in the orbit as our symbol probabilities we have,

$$
P\left(\alpha_{i}\right)=\frac{1 / d_{i}}{\sum_{j} 1 / d_{j}} .
$$

We'd also like an analog of the proportion spread from the binary development above; e.g. loosening fully regular sequences from $\overline{a b c}$ and cyclic permutations, to sequences that allow more variation in the proportion of a given symbol within any block of a given length. This is accomplished by adjusting the height, $h$, of the initial bounding simplex (analogous to extending the initial length of the line segment under fixed step sizes in the binary case, denoted there by $s$ ).

We however require $h$ to be large enough that there exists at least one infinite shift sequence $\Psi_{i_{1}}, \Psi_{i_{2}}, \ldots$ so that $\left(X_{t}\right)_{t \geq 1}$ does not vanish (i.e. so its altitude does not go to zero). The minimal $h, h_{\text {min }}$, that satisfies this requirement will correspond to the lowest possible level of proportion spread. That such an $h_{\text {min }}$ exists follows 
from noting that each $\Psi_{i}$ removes at most $d_{i}$ from the altitude (we can think of the shift as removing at most $d_{i}(k-1)$ times the unit thickness, $1 /(k-1)$, from all but face $\alpha_{i}$; as the shifts go on, not all shifts affect all $k-1$ opposing faces, but assuming so suffices for a bound)-so $h>\sum_{i} r_{l}$ will suffice. Increases in units of $1 /(k-1)$ from the minimum altitude will broaden the set of sequences considered fully regular.

As an example, consider again the three symbol alphabet $\{a, b, c\}$, under an equiprobable distribution. This requires $d_{1}=d_{2}=d_{3}=1$, by (7.1). Since the dimension is 2 , the simplex altitudes will be discretized into units of $1 / 2$. The first shift in any direction will remove an altitude of 1 (the two opposing faces are each sheared by the amount $1 / 2$ ). The next shift may be either made in the same direction, for a total altitude loss of 2 , or can be made in either of the two remaining directions, for an altitude loss of $1 / 2$ (only one face will lose a thickness of $1 / 2$ ). Note at this point cyclic shifts $(a \rightarrow b \rightarrow c$ and permutations) will prevent further altitude loss. The minimal height in this case is $h_{\min }=2$, which amounts to four units each of size $1 / 2$. The triangular simplex in Figure 11 depicts this case. The initial shift is along the unit normal to the $a$ face. Subsequent shifts $b \rightarrow c \rightarrow a \ldots$ or $c \rightarrow b \rightarrow a \ldots$ will prevent the simplex orbit from vanishing; there are no other choices. This makes $h=2$ and the corresponding $a b c \ldots$ (and permutations of $a b c$ ) the minimal fully regular sequences.

7.2. Matrix representations of simplex shifts. We note the unit shear cells of a regular $(k-1)$-simplex of height $h=u /(k-1)$ can be created from a series of at most $k, \Psi_{\alpha}$ shear operations: we shear from each face, $\alpha_{i}$, the calibrated amount $y_{i} /(k-1), y_{i} \in \mathbb{N}$, to arrive at the desired unit cell (consider the process in 2 dimensions-see again Figure 11). This is analogous to assigning altitude coordinates to each such cell, the set of perpendicular distances between the parallel faces $\alpha_{i}$ of boundary $B$ and face $\alpha_{i}$ of the unit shear cell. This produces the $k$-tuple $\left(a_{1}, \ldots, a_{k}\right)$, $a_{i} \in[0, u-1]$, with some joint restrictions on the $a_{i}$. The restrictions follow from an extension of Viviani's Theorem, namely that for any regular polyhedron the sum of distances from any interior point to the outer faces is independent of the point's location. This produces the coordinate set,

$$
\left\{\left(a_{1}, \ldots, a_{k}\right) \mid a_{1}+\ldots+a_{k}=u-1, a_{i} \geq 0\right\} .
$$

We can easily determine the number of unit cells. The fact the $a_{i}$ can be 0 allows treating the sum condition, $a_{1}+\ldots+a_{k}=u-1$, as a weak composition; i.e. the number of ways to sum exactly $k$ integers $\geq 0$ to value $u-1$ :

$$
\text { (\# of unit shear cells for a }(k-1) \text {-simplex, } h=u /(k-1))=\left(\begin{array}{c}
u+k-2 \\
k-1
\end{array}\right) \text {. }
$$

The effect of a shift on face $\alpha_{i}$ by amount $d_{i} \in \mathbb{N}$ on any unit cell readily translates into the $a_{i}$ coordinates:

$$
d_{i} \Psi_{i}\left(a_{1}, \ldots, a_{k}\right)=\left(a_{1}-d_{1}, \ldots, a_{i}+d_{i}(k-1), a_{i+1}-d_{i+1}, \ldots, a_{k}-d_{k}\right) .
$$

By construction, shifts $d_{i} \Psi_{i}$ map unit cells to unit cells (with the possibility a cell is mapped to null, that is shifted outside $B$ ).

Define the 'simplicial vector' $\mathbf{v}$ as the vector whose $j$ th entry assigns weight $v_{j} \in \mathbb{R}$ to the $j$ th simplex unit cell, under some coordinate basis.

Each directional $\Psi_{i}$ shift of the simplex can then be represented as a binaryvalued matrix, $S_{i}$, acting on the simplicial vector. In parallel with the binary symbol 
case, define the set of fully regular sequences of length $t$ as surviving prefixes of infinite-surviving paths,

$$
\operatorname{Reg}(t)=\left\{\alpha_{i_{1}}, \ldots, \alpha_{i_{t}} \mid\left\|\cdots S_{i_{t+1}} S_{i_{t}} \cdots S_{i_{1}}\right\|_{e n}>0 \text { for some } i_{t+1}, i_{t+2}, \ldots\right\}
$$

We are interested in deriving the binary matrix forms for the $\Psi_{\alpha}$ linear shift operators. This may be accomplished by describing all unit cells in terms of their coordinates $\left(a_{1}, \ldots, a_{k}\right)$, and for a given shift, $\Psi_{i}$, applying the action of $(7.2)$ to each. Cells that map outside the boundary will have $a_{j} \notin[0, u-1]$, and can be ignored; all others will map from one coordinate $k$-tuple to some other. Under any enumeration of the $k$-tuples, 1 to $\left(\begin{array}{c}u+k-2 \\ k-1\end{array}\right)$, this generates ordered pairs $\left(\hat{e}_{c}, S_{i} \hat{e}_{c}\right)$, where $\hat{e}_{c}$ is a standard basis element corresponding to the $c$ th unit shear cell under the given enumeration. If $S_{i} \hat{e}_{c}=\hat{e}_{r}$, then this amounts to assigning a 1 to the $(r, c)$ th entry of $S_{i}$. All such pairs completely determine the $S_{i}$ matrix.

Continuing with the three-symbol-equiprobable example with height $h=2$ (referring once again to Figure 11), if we enumerate the unit cells starting with 1 at the lower left, $c a$ vertex, then proceed left-to-right, from the lowest row to the highest, the simplex shift operator $\Psi_{a}$ can be coded as a (sparse) 10x10 matrix with entries $S_{a}(8,2)=S_{a}(9,3)=S_{a}(10,6)=1$, with all other entries 0. Similarly, $S_{b}(1,6)=S_{b}(2,7)=S_{b}(5,9)=1$, and $S_{c}(3,5)=S_{c}(4,6)=S_{c}(7,8)=1$, with all other entries 0 .

A few things are worth noting.

The shift matrices can be cast in any enumeration basis of the unit cells. Any representation will produce the same results with respect to survivability of a sequence, $\cdots S_{i_{2}} S_{i_{1}}$. To change a shift matrix from one cell-enumeration to another, we simply use the corresponding permutation change of basis:

$$
S_{i} \mathbf{v}_{1}=\Phi^{-1} S_{i} \Phi \mathbf{v}_{2}
$$

where $\mathbf{v}_{1}$ is in the old enumeration, and $\mathbf{v}_{2}$ is in the new enumeration, and $\Phi$ is the permutation matrix that negotiates between the two.

The simplex shift operators in general may be quite sparse. In the ongoing three-symbol example, there are 3 zero-columns, corresponding to cells 1, 4, 10 (the tip cells), all of which are mapped to zero after any one of the $S_{\alpha}$ have acted. In general, zero-columns within an $S_{i}$ map a corresponding vector basis element to null- that is they represent a cell mapped outside the bounding simplex, and zerorows correspond to cells that no other cell maps into. By extension, zero-columns and -rows of the sum, $\sum_{i} S_{i}$, correspond to overall null mappings of certain cells. These null mappings amount to dead ends in the $S_{\alpha}$ product chains. We note such null cells are unique to higher dimensions, $k \geq 3$ : in the 1-dimensional simplex case (a line segment), all 'unit cells' mapped to another, under either a shift right, or shift left along the line segment.

In aim of preserving only the infinite template sequences of Reg in (7.3), any zero-columns and zero-rows common to all the $S_{\alpha}$ will be removed. Any such dead end cells will of course be encountered on some periodic product chain when the simplex height is made sufficiently larger. The elimination of null cells has the added benefit of reducing the total number of cells in play. Without any degree of reductions, the matrix dimensions would otherwise grow quite rapidly with the size of the symbol alphabet $|\mathcal{A}|$ : for a 4 -symbol simplex with altitude 18 units $(h=6)$, the simplicial vector has 1140 components, requiring initial shift matrices of equally large dimensions. 
Accordingly, the simplicial shift matrices will be processed in two steps: a reduction stage, and a route finding stage.

In the reduction stage, the mutually $S_{\alpha}$-null components are removed. This is done by considering the sum $\sum_{i} S_{i}$, and removing all columns composed entirely of 0 's, and removing their corresponding rows; we also remove all zero rows, and all their corresponding columns. This process is then repeated, until we have a reduced set of shift matrices which jointly (their sum) has least one 1 on each row and each column.

In the three-symbol example, 7 cells survive after any of the first shifts, and 6 survive after two shifts. At this point there are no more zero columns or zero rows to remove, and we have,

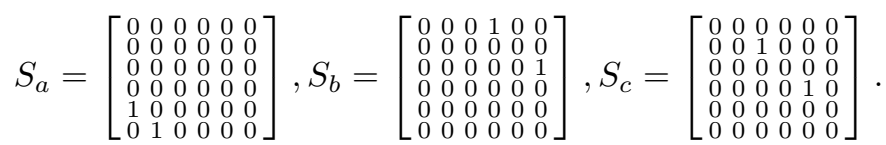

The fact the reduced matrices have no zero rows or zero columns jointly, indicates that any standard basis vector $\hat{e}_{j}=[0 \ldots 010 \ldots 0]^{\top}$ is shifted to one or more different $\hat{e}_{k}$ by one or more $S_{i}$ (by nature of the $S_{\alpha}$ shifting, it cannot be mapped to itself). Iterating, this produces one or more sets of linked basis vectors. Finding these sets of connected basis elements constitutes the route finding stage.

Continuing with the three-symbol example, the matrices separate into two simple basis vector routes. Re-enumerating coordinates, this produces a final blockdiagonal form for the $S_{\alpha}$ :

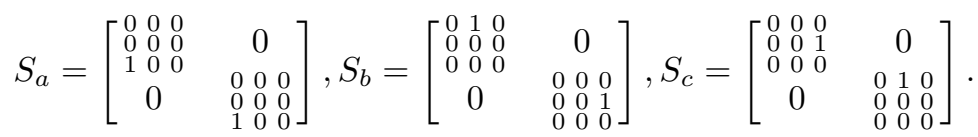

Each of the two block rows correspond to distinct sets of linked basis vectors.

Denoting the 2! circular permutations of the 3 -symbol string, $a b c$, by $a b c$ and $a c b$, the shift matrices simply test a given sequence for membership in the class of contiguous substrings of $\overline{a b c}$ and $\overline{a c b}$. The top and bottom $3 \times 3$ block rows test for membership in the first and second classes respectively; membership in either will produce a non-vanishing norm of $S_{\alpha}$ products.

This simple format for the $S_{\alpha}$ is easily extensible to any equiprobable $k$-symbol set, $P(a)=P(b)=\ldots=1 / k$, at minimal simplex height $h_{\min }$. We construct the $k$ by $k$ matrix set $\left\{D_{1}, \ldots, D_{k}\right\}$, where each $D_{i}$ 's entries are zero but for a single 1 at a row and column different from all the other $D_{j}$ in the set. The block diagonals for the $S_{\alpha}$ are then constructed by, at each collective block row, taking a unique circular permutation of the ordered $k$-tuple $\left(D_{1}, \ldots, D_{k}\right)((k-1)$ ! in all $)$ and assigning its elements to the corresponding block row of $S_{1}, S_{2}, \ldots$, in order.

7.3. Block rows as graphs. In general, each block row of the resulting, reduced, post-reduction and post-route-finding $S_{\alpha}$ generates a directed graph with edges labeled by the $\alpha_{i}$. Each $S_{\alpha}$ sequence permitted by that block row is reflected in a particular directed path on the graph. The $r, c$ th entry (of a 1 ) in a given $S_{i}$ can be read as a path from vertex $c$, as standard basis element $\hat{e}_{c}$, to vertex $r$, as $\hat{e}_{r}$, with the edge labeled by $\alpha_{i}$. The order the vertices occur will be the reverse order of the left-acting matrix product. 
The adjacency matrix associated with the graph is given by

$$
A d j=\left(\sum_{i} S_{i}\right)^{\top}
$$

where ${ }^{\top}$ denotes the transpose (a 1 entry at $(r, c)$ in an adjacency matrix denotes an edge from vertex $r$ to vertex $c$ ). Note the adjacency matrix loses the edge label information.

The graph then is edge-labeled (vertex labels do not matter), and simple (no edges connecting a vertex to itself), since by nature of the simplex shifts we cannot map a unit shear cell into itself.

Since the route finding step amounts to finding sets of connected vertices, and the reduction stage ensured a 1 in every row and column (other than the diagonal), Adj must be strongly irreducible. Strong irreducibility guarantees the graph is strongly connected: between any two vertices there are at least two directed paths, one in each direction. We recall that a cycle in a directed graph amounts to a closed directed path that encounters no vertex more than once. Since in a strongly connected graph every edge is part of a cycle, we will see in Section 7.5 the block row graphs may be viewed as a collection of cycles, each of length some integer multiple of a basic period $(\tau)$.

As an example, a more complicated case occurs under the same 3 -symbol conditions when $h$ is increased from 2 to 3 . This produces final $S_{\alpha}$ of size 18 by 18, decomposable into block diagonals of three $6 \times 6$ matrices each. Denote for the $i$ th block row the block matrix elements $\left\{D_{i, 1}, D_{i, 2}, D_{i, 3}\right\}$. Since the symbols are equiprobable, the amount the 2 -simplex is shifted by each one is equal (to 1 ). So by symmetry arguments, under some permutation on symbols any $D_{i, j_{1}}$ is coordinatepermutation-similar to a block matrix in the other two rows $\left(j \neq j_{1}\right)$. We'll examine the first block row of the $S_{\alpha}$ matrices:

$$
D_{1 a}=\left[\begin{array}{cccccc}
0 & 0 & 0 & 0 & 0 & 0 \\
0 & 0 & 0 & 0 & 0 & 0 \\
0 & 0 & 0 & 0 & 0 & 0 \\
1 & 0 & 0 & 0 & 0 & 0 \\
0 & 1 & 0 & 0 & 0 & 0 \\
0 & 0 & 1 & 0 & 0 & 0
\end{array}\right], D_{1 b}=\left[\begin{array}{llllll}
0 & 0 & 1 & 0 & 0 & 0 \\
0 & 0 & 0 & 0 & 0 & 0 \\
0 & 0 & 0 & 0 & 1 & 0 \\
0 & 0 & 0 & 0 & 0 & 1 \\
0 & 0 & 0 & 0 & 0 & 0 \\
0 & 0 & 0 & 0 & 0 & 0
\end{array}\right], D_{1 c}=\left[\begin{array}{llllll}
0 & 0 & 0 & 0 & 0 & 0 \\
0 & 0 & 1 & 0 & 0 & 0 \\
0 & 0 & 0 & 1 & 0 & 0 \\
0 & 0 & 0 & 0 & 0 & 0 \\
0 & 0 & 0 & 0 & 0 & 1 \\
0 & 0 & 0 & 0 & 0 & 0
\end{array}\right]
$$

The surviving sequences these induce can be seen by generating the associated directed, strongly connected graph. See Figure 12. Each node has been numbered according to the matrix's effect on the standard basis vectors: e.g. $D_{1 a} \hat{e}_{1}=\hat{e}_{4}$ (the leftmost edge of the graph).

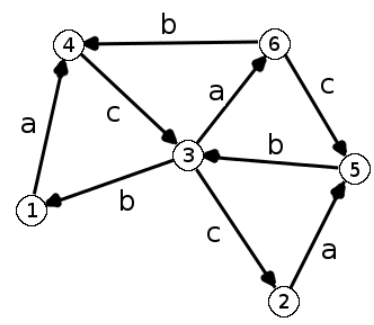

FIGURE 12. directed graph, derived from the matrices $D_{1 \alpha_{j}}$, showing allowed cycles 
We can read the allowed sequences off the graph. As expected, increasing the height $h$ from 2 to 3 expands the sequences considered fully regular, from strict $\overline{a b c}$ and $\overline{a c b}$ to sequences such as $\overline{b b a c c a}$ (noting again the sequence-order-as-matrixmultiplication reads from right to left).

By the symmetric nature in the equiprobable case, where all simplex shifts are of equal magnitude 1 (in two dimensions, two units of $1 / 2$ ), we would expect the graph sets to accommodate all permutations on symbols-a completeness under automorphisms and isomorphisms (under the labeled edges; node numbering is arbitrary under the choice of simplex cell enumeration). We see the graph in Figure 12 has non-trivial automorphism $a b c \rightarrow a c b$ (flip the graph 180 degrees about a line connecting nodes 3 and 6 ). The other two block rows have graphs isomorphic to the first block row's, under symbol swaps $b \leftrightarrow a$, and $a \leftrightarrow c$. These, combined with the corresponding automorphisms, account for all six permutations of the symbol set $\{a, b, c\}$.

7.4. Relations to sequence discrepancy and fairness. The homogeneity or evenness in the distribution of symbols in a sequence is of interest in diverse areas such as operations analysis [28, 64], dynamical systems [12], and quasirandom sequences, these last useful for optimizing numerical integration techniques. Since the $S_{\alpha}$, and their controllable 'proportion spread,' have some relevance in this context, it's worth exploring these connections in a little more detail. This also allows obtaining an upper bound on the minimal simplex height, $h_{\min }$, associated with a given shift-distance set, $\left\{d_{1}, \ldots, d_{k}\right\}$.

Given sequence $S$, define $Z_{i}(t)$ as the number of occurrences of symbol $\alpha_{i}$ in the first $t$ positions of $S$. Then define the discrepancy of symbol $\alpha_{i}$ at prefix length $t$ as,

$$
D_{i}(t)=Z_{i}(t)-p_{i} t,
$$

where $p_{i}, \sum_{i} p_{i}=1$, are the desired rates of the corresponding symbols. Fair sequences are then generally defined in relation to the $k$ discrepancy values, taken over all prefixes $t$, up to some fixed length $l$. Specific approaches include minimizing the sum of discrepancy scores over all $i$ and all prefixes ('min-sum'), or finding sequences that minimize the maximum discrepancy, again taken over all $i$ and all prefixes ('min-max'). In practical applications, there also may be a convex wrapper function for each $i$, so that the goal is to minimize sets of $f_{i}\left(D_{i}(t)\right)[28,64,54]$.

Here the discrepancy of interest will be the 'min-max' form. That is, we consider fair sequences those with minimal $V(S)$, where,

$$
V(S) \equiv \max \left\{\left|Z_{i}(t)-p_{i} t\right|: \forall i \in[1, k], \forall t \in[1, l]\right\} .
$$

with $l$ the sequence length, $p_{i} \in \mathbb{Q}$.

Candidates for fair sequences under the simplex method are any sequences whose corresponding $S_{\alpha}$ product chain is nonzero. Once the $S_{\alpha}$ matrices have been derived, we select a block row (if there is more than one), and proceed with symbol choices that maintain a nonzero result. Such sequences may be visualized by consulting the corresponding graphs, such as in Figure 12. We may also consider a 'cyclic word' as a sequence whose last symbol has a directed graph edge leading to the first symbol; such a word may be repeated indefinitely, forming a periodic sequence of arbitrary length; any prefix may then be taken, as with the binary case. 
In general, given symbol sequence $S=\alpha_{i_{1}}, \ldots, \alpha_{i_{m}}$, we would expect the measure of the breadth or volume, consumed by the associated $k-1$ dimensional walk with steps $d_{i_{1}} \mathbf{n}_{i_{1}}, \ldots, d_{i_{m}} \mathbf{n}_{i_{m}}$ to be related to the discrepancy of the sequence. That is, the minimum height,

$$
\min _{h}\left|\sum_{j=1}^{s} d_{i_{j}} \mathbf{n}_{i_{j}} \in B_{h}\right| s \leq m
$$

where $B_{h}$ is a regular simplex of height $h$, should correspond in some way to the min-max discrepancy of sequence $S$.

Recall from Section 7.2 that a unit shear cell under shifts can be referenced by its altitude coordinates $\left(a_{1}, \ldots, a_{k}\right), a_{i} \in[0, u-1]$, where $\sum_{i} a_{i}=u-1$. We can translate the shift walk for the action of sequence $S$ on a unit cell at coordinates $\left(c_{1}, \ldots, c_{k}\right)$ into altitude vector form:

(7.6)

$$
\left[\begin{array}{c}
c_{1} \\
c_{2} \\
\vdots \\
c_{k}
\end{array}\right]+Z_{1}(t)\left[\begin{array}{c}
(k-1) d_{1} \\
-d_{1} \\
\vdots \\
-d_{1}
\end{array}\right]+Z_{2}(t)\left[\begin{array}{c}
-d_{2} \\
(k-1) d_{2} \\
-d_{2} \\
\vdots
\end{array}\right]+\ldots+Z_{k}(t)\left[\begin{array}{c}
-d_{k} \\
\vdots \\
-d_{k} \\
(k-1) d_{k}
\end{array}\right]
$$

The resultant vector gives the position of the unit shear cell at the end of the walk, in coordinate units of $1 /(k-1)$. Note for the unit shear cell to 'survive' the walk of length $t$ on a simplex of height $h=u /(k-1)$, we require, for each $i$ th component,

$$
c_{i}+Z_{i}(s)(k-1) d_{i}-\sum_{j \neq i} Z_{j}(s) d_{j} \in[0, u-1],
$$

for all $s \leq t$. Rewriting (7.6) in matrix form we have,

$$
\mathbf{C}+\Delta \mathbf{Z} \equiv\left[\begin{array}{c}
c_{1} \\
c_{2} \\
\vdots \\
c_{k}
\end{array}\right]+\left[\begin{array}{cccc}
(k-1) d_{1} & -d_{2} & \cdots & -d_{k} \\
-d_{1} & (k-1) d_{2} & \cdots & -d_{k} \\
\vdots & & & \vdots \\
-d_{1} & -d_{2} & \cdots & (k-1) d_{k}
\end{array}\right]\left[\begin{array}{c}
Z_{1} \\
Z_{2} \\
\vdots \\
Z_{k}
\end{array}\right]
$$

From (7.4), we may write $Z_{i}(t)=p_{i} t+D_{i}(t)$. Note that $\Delta\left(t\left[p_{1}, \ldots, p_{k}\right]^{\boldsymbol{\top}}\right)=\mathbf{0}$, by (7.1), and since $\Delta\left[1 / d_{1}, \ldots, 1 / d_{k}\right]^{\top}=\mathbf{0}$. Applying this to (7.6) we obtain

$$
\mathbf{C}+\Delta \mathbf{Z}=\mathbf{C}+\Delta \mathbf{D},
$$

where $\mathbf{D}=\left[D_{1}, \ldots, D_{k}\right]^{\top}$. Considering (7.7) with (7.8), we now have a form directly linking the simplex height to sequence discrepancy.

The algorithm developed above for creating the $S_{\alpha}$ for each instance of a shift set, $\left\{d_{1}, \ldots, d_{k}\right\}$, required a minimal $h_{\min }$ so that at least one unit shear cell survived. We would like to know something about such a minimum, as well as more precisely how this translates into the discrepancy, $D_{i}$, for any individual symbol. Two remarks are in order.

First, the minimal height is easy to calculate for the equiprobable case, where $d_{i}=1$ for all $i$. The first shift costs altitude 1 . The next obvious choice to minimize altitude loss is any direction but the first, for a loss of $(k-2) /(k-1)$ (the shortened simplex now sheared of $k-2$ faces). Continuing gives $h_{\min }=k / 2+1 /(k-1)$. In the general case, however, the walks can be quite complex. 
Second, simplex height gradations naturally give rise to a nested symbolic sequence discrepancy hierarchy. Let $R_{h}$ denote the set of all admissible infinite sequences corresponding to simplex height $h$. Let $R_{h_{\text {min }}}$ denote the set of all sequences corresponding to minimal simplex height $h_{\min }$; i.e. the smallest height that still allows at least one surviving sequence under the simplex shift dynamic. Then

$$
R_{h_{\min }} \subseteq R_{h_{\min }+1 /(k-1)} \subseteq R_{h_{\min }+2 /(k-1)} \subseteq \ldots \subseteq R_{h_{\min }+j /(k-1)} \subseteq \cdots
$$

This follows since every sequence with some surviving unit shear cell under simplex height $h_{1}$ will also clearly survive under any simplex height $h_{2}>h_{1}$.

We then have,

Lemma 1. Infinite sequence $S$ is in some $R_{h_{\min }+j /(k-1)}$ iff $\left|D_{i}(t)\right| \leq M<\infty$ for all $i \in[1, k]$ and all $t \geq 1$.

Proof. First note the algorithm developed above is exhaustive, in that every sequence that induces a walk that remains in an $R_{h}$ will be included. Now if $\left|D_{i}(t)\right|$ is bounded for all $i$ and $t$, then the vector $v=\Delta \mathbf{D}(t)$ is clearly bounded as well. Then by (7.7) and (7.8), there must exist some $u$ such that each element in $\mathbf{C}+v$ lies in the range $[0, u-1]$ for all $i$ and $t$ as well. Since $R_{u(k-1)}$ is assured of containing all such walks, this proves the $\Leftarrow$ direction. For $\Rightarrow, S$ 's membership in $R_{h_{\min }+j /(k-1)}$ gives,

$$
\mathbf{C}+\Delta \mathbf{D} \in\left[\begin{array}{c}
{[0, u-1]} \\
\vdots \\
{[0, u-1]}
\end{array}\right],
$$

for all $t$, with $u=(k-1) h_{\min }+j$. Suppose $D_{i}(t)$ were not bounded for some $i^{\prime}$ and some $t_{0}$. Then $\mathbf{C}+\Delta \mathbf{D}\left(t_{0}\right)$ is unbounded in its $i^{\prime}$ th component. Its walk then can't be contained in any simplex of finite height, a contradiction.

The nested hierarchy of (7.9) then provides an ordering by simplex height of every infinite sequence with bounded discrepancy under the definition of (7.5). All such sequences may be generated from the $S_{\alpha}$ matrices and their block rows (or, equivalently, the corresponding graphs).

Next, a lemma on the monotonicity of the simplex height as a function of the $\left|D_{i}\right|$. It is immediately followed by a claim regarding the sequences of minimal discrepancy, i.e. those with some optimally small $V(S)$, as defined in (7.5).

Lemma 2. Let

$$
E_{M}=\left\{S:\left|Z_{i}(t)-p_{i} t\right| \leq M, \forall t \geq 1, \forall i\right\} .
$$

Then for $M_{1} \leq M_{2}$, if $E_{M_{2}} \subset R_{h_{\min }+j /(k-1)}$ for some $j$, then $E_{M_{1}} \subset R_{h_{\min }+j^{\prime} /(k-1)}$ for some $j^{\prime} \leq j$.

Proof. That $E_{M_{1}}$ is in some $R_{h_{\min }+j /(k-1)}$ follows from Lemma 1 . Let $\varepsilon_{i}=Z_{i}(t)-$ $p_{i} t$, and consider the $i$ th element of vector $\Delta \mathbf{Z}(t)$ :

$$
v_{i}=\varepsilon_{i}(k-1) d_{i}-\sum_{j \neq i} \varepsilon_{j} d_{j}
$$

We have for sequences $S_{1} \in E_{M_{1}}$ and $S_{2} \in E_{M_{2}},\left|v_{1, i}\right| \leq\left|v_{2, i}\right|$, since $\left|\varepsilon_{1, i}\right| \leq\left|\varepsilon_{2, i}\right|$ for all $i$ by definition of the $E_{M}$. Since the magnitudes of each component of the $\Delta \mathbf{D}(t)$ vector for $S_{1}$ are pairwise less than or equal to those for $S_{1}$, the minimal simplex height required to contain the walk of sequence $S_{1}$ is then less than or equal 
to the height required to contain $S_{2}$. By definition of the $R_{h_{\min }+j /(k-1)}$, the proof is complete.

Claim 5. All sequences of minimal discrepancy under the definition of (7.5) must lie in $R_{h_{\min }}$, the set of sequences permitted under the simplex of minimal height.

Proof. Suppose $S_{0}$ under $k$ symbols and some $\left\{p_{i}\right\}$ has minimal discrepancy: $V\left(S_{0}\right) \leq V(S), \forall S$. By Lemma 1 we know it must lie in some $R_{h_{\min }+j /(k-1)}$. Now suppose $S_{0}$ is in some $R_{h_{\min }+j_{0} /(k-1)}$, but not in $j<j_{0}$. By $V\left(S_{0}\right)$ 's minimality, we know $S_{0}$ lies in $E_{M_{0}}$, and any $S$ lies in some $E_{M}$, where $M_{0} \leq M$. By Lemma 2, we have $S_{0} \in R_{h_{\min }+j_{0} /(k-1)}, S \in R_{h_{\min }+j /(k-1)}$, with $j_{0} \leq j$, a contradiction.

We are now in a position to estimate the minimal simplex height, conditional on knowing a bound on the sequences with minimal discrepancy. The following theorem is from [55] and [39]:

Theorem 3. Given $k$ symbols and their associated probabilities, $\left\{p_{i}\right\}$, there exists an infinite sequence such that

$$
\left|Z_{i}(t)-p_{i} t\right| \leq 1-\frac{1}{2(k-1)}
$$

for all $i$, and all $t \geq 1$.

Knowing by Claim 5 the optimal of such minimal sequences must lie in $R_{h_{\min }}$, we consider the vector $\Delta \mathbf{D}$ when the $D_{i}$ obey the discrepancies of (7.10). The $i$ th element of the vector is,

$$
v_{i}=\varepsilon_{i}(k-1) d_{i}-\sum_{j \neq i} \varepsilon_{j} d_{j}
$$

where $\left|\varepsilon_{i}\right| \leq 1-1 /(2(k-1))$ for all $i$. If $D_{\min }$ is the bound of Theorem 3 , we have the bounding interval,

$$
v_{i} \in\left[-D_{\min }\left((k-1) d_{i^{\prime}}+\sum_{j \neq i^{\prime}} d_{j}\right), D_{\min }\left((k-1) d_{i^{\prime}}+\sum_{j \neq i^{\prime}} d_{j}\right)\right],
$$

for all $t$. By (7.8), the interval translates into the maximum necessary range of altitude coordinates needed to accommodate a walk for optimal sequences of (7.10). So we have the bound

$$
h_{\min } \leq \frac{1}{k-1}\left(2 D_{\min }\left((k-1) d_{i^{\prime}}+\sum_{j \neq i^{\prime}} d_{j}\right)+1\right) .
$$

For further ideas on bounding $h_{\min }$, the reader may wish to consult [3], and the Steinitz Lemma. The Lemma concerns a finite set of vectors, $v_{1}, \ldots, v_{n}$ whose total sum is zero. Of interest is the optimal ordering of the vectors so that the partial sum,

$$
\sum_{j=1}^{l} v_{i_{j}}
$$

remains in some minimal normed volume for all $l \in[1, n]$.

Since the action of the simplex shifts may be represented as a sum of vectors, as in (7.6), in bounding $h_{\min }$ we are effectively asking about the minimal volume required to contain at least one infinite partial sum over the shift vectors. By 
finiteness of the number of cells available, every infinite route must eventually fold back on itself. As a result, we can confine the question to the minimal volume required of a single return orbit (which finite vector set sums to zero). This puts the problem almost exactly in the terms of the Steinitz Lemma. The difference is in the types of volumes-we have a simplicial volume here, while Steinitz's Lemma gives its bound in terms of (normed) spherical volumes.

7.5. Graph properties and Christoffel extensions; cylinder graphs. In this section we'll gain a little more insight into the types of sequences specific to a given $R_{h_{\min }+j /(k-1)}$, by considering the sequence graphs (see for example Figure 12).

Recall from Section 7.3 that the graph associated with any $S_{\alpha}$ block row is directed, simple, and strongly connected. By strong connectivity, recall we also have that every edge is part of a cycle.

In practice, the graphs corresponding to any $S_{\alpha}$ block row even at the minimal $h_{\text {min }}$ may have multiple edges leaving or entering any given vertex (this is not always the case; for example equiprobable sequences have very simple shift matrices: $(k-1)$ ! block rows corresponding to all possible cyclic permutations on the symbols, each block row with a graph with only a single edge entering and leaving each vertex). This creates choices, or branches, that still keep the orbit of the unit cells in question within the $h$ boundary, but can vary in their $V(S)$ discrepancy values.

We observe that any closed orbit obeys $S_{i_{t}} \cdots S_{i_{1}} v=v$ for some non-zero $v$. This amounts to a sequence that eventually maps one or more unit shear cells into themselves. In altitude coordinates, this is equivalent to $\mathbf{C}+\Delta \mathbf{w}=\mathbf{C}$, for some set of steps encoded in $\mathbf{w}$. Of interest, then, is the null space of $\Delta$. The null space follows from the previous observation $\Delta\left[1 / d_{1}, \ldots, 1 / d_{k}\right]^{\top}=\mathbf{0}$, and the fact $\Delta$ is of rank $k-1$. Since the $Z_{i}$ are limited to integer values, this gives,

$$
\operatorname{null} \Delta=j r_{l}\left[\begin{array}{c}
\frac{1}{d_{1}} \\
\vdots \\
\frac{1}{d_{k}}
\end{array}\right]
$$

where $r_{l}=\operatorname{lcm}\left\{d_{1}, \ldots, d_{k}\right\}$ (recall $\left.\operatorname{gcd}\left\{d_{1}, \ldots, d_{k}\right\}=1\right)$. The closed orbits are then of length $j \tau, j \geq 1$, where

$$
\tau=r_{l} \sum_{i} \frac{1}{d_{i}} .
$$

By construction under the associated algorithm, since each block row of the $S_{\alpha}$ in any $R_{h_{\min }+j /(k-1)}$ may be regarded as a set of cycles, each of the cycles must have length $j \tau, j \geq 1$.

At the minimal height simplex, by analogy with the binary alphabet case, we can now define a natural geometric extension of Christoffel words. The set of $k$ symbol Christoffel extensions consist of all allowed periodic sequences under $h_{\min }$. They are each of some period $j \tau, j \in[1, \infty)$. (For other approaches to extending Christoffel words to higher dimensions, see [29]. The authors base their extension on certain symmetry and conjugate properties of binary Christoffel words, the result of a theorem of Pirillo. We note that for this paper's geometric extension, even simple cases such as, $k=3$, with shift distance set $\{1,2,3\}, h_{\min }=5$, do not obey the conjugate property.)

While it is conjectured that a minimal cycle of length $\tau(j=1)$ exists for all such Christoffel extensions, we are assured there exists at least one such minimal 
period sequence in the block row set. By the nesting property, (7.9), this readily extends to all the $R_{h_{\min }+j /(k-1)}$ graph sets:

Claim 6. Every $R_{h_{\min }+j /(k-1)}$ must have at least one graph that contains a closed orbit of length $\tau$.

Proof. We first show this must be true for $R_{h_{\min }}$. From (7.12), we have a closed orbit of length $\tau$ iff the proportion of each symbol $i$ obeys $Z_{i}^{c}(\tau)=p_{i} \tau$ (letting the $c$ superscript denote $Z_{i}$ that reach closed orbit values at $\tau$ ). Suppose no graph in $R_{h_{\min }}$ allowed a closed orbit of length $\tau$. Then any sequence of length $\tau$ generated by the graph must have two or more symbols that don't obey the ideal proportions; that is,

$$
\left|Z_{i_{j}}(\tau)-Z_{i_{j}}^{c}(\tau)\right| \geq 1
$$

for at least two $i_{j} \in[1, k]$. Then it follows that for some $t \leq \tau$,

$$
\left|Z_{i_{j}}(t)-p_{i_{j}} t\right| \geq 1
$$

(since $Z_{i}^{c}(\tau)-p_{i} \tau=0$ by construction). But by Theorem 3, and Claim 5 , the graphs of $R_{h_{\min }}$ must generate at least one sequence with $V(S) \leq 1-1 /(2(k-1))$, a contradiction.

By the $R$ nesting property, (7.9), this proves the claim.

To better understand the graphs produced by the $S_{\alpha}$ block rows, we next define a standard cylindrical form.

Let a stepwise cylinder graph consist of $\tau$ sets of nodes, $n_{1}, \ldots, n_{\tau}$. Each node in each $n_{i}$ may have up to $k$ uniquely labeled directed edges entering it, and up to $k$ uniquely labeled directed edges leaving it. Let $t$ be the 'time' step, indexing the $\tau$ node sets. Then every directed edge leaving a node at time step $t$, must enter a node at time step $t \bmod (\tau)+1$. The resulting graph may be drawn on a cylinder; all nodes at time $t=\tau$ have directed edges leading back to nodes at the first time step.

Since every graph is decomposable into some set of directed-edge closed loops, each with period some multiple of $\tau$, any graph may be projected onto a sequence of $\tau$ steps. This results from the ability to project any loop of period $j \tau, j>1$, onto a stepwise cylinder graph of period $\tau$. See Figure 13. The time-step node sets, $n_{1}, n_{2}, n_{3}$, have been depicted as vertical columns.

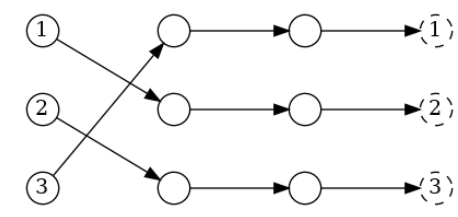

FigURE 13. projecting an orbit of length $3 \tau$ onto a $\tau$ stepwise cylinder graph; nodes with dashed outlines in rightmost column depict the cylindrical wraparound

In Figure 14, the example graph of Section 7.3 has been redrawn as a stepwise cylinder graph.

In general, there may or may not be paths of period longer than $\tau$. In the graph of Section 7.3, there are none longer than $\tau=3$; this is obvious from the bottleneck of the single node at the third time step. 


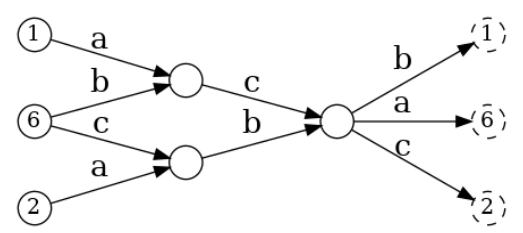

FiguRE 14. the 3-symbol, equiprobable graph of Figure 12 rearranged as a cylinder graph, of period $\tau=3$

\begin{tabular}{llc}
$\left\{d_{1}, \ldots, d_{k}\right\}$ & $h_{\min }$ & $S_{\alpha}$ block rows \\
\hline $1,2,3$ & 5 & $15 \times 15(\mathrm{x} 2)$ \\
$2,3,5$ & 9 & $46 \times 46,47 \times 47$ \\
$4,7,11$ & 21 & $248 \times 248(\mathrm{x} 2)$ \\
$1,2,3,5$ & $28 / 3$ & $172,175,176,177(\mathrm{x} 2), 180$ \\
$2,2,5,5$ & $26 / 3$ & $16 \times 16(\mathrm{x} 2)$ \\
$3,5,7,8$ & $64 / 3$ & $2039,2042,2043(\mathrm{x} 3), 2044$ \\
& &
\end{tabular}

7.6. Discrepancy examples. In Figure 15 are scatter plots for the maximum discrepancies, $V(S)$ (7.5), of $S_{\alpha}$ product chains of length 1e4, chosen uniformly from a given $R_{h_{\min }}$. Table 2 shows the $S_{\alpha}$ characteristics.

The stratification of values in the $\{1,2,3\}$ and $\{2,2,5,5\}$ cases are the result of the relatively few positions the unit shear cells take in any given orbit: consulting (7.8), note the discrepancy values take unique values for every altitude coordinate $k$-tuple. The relatively low simplex height, and correspondingly small block row matrix dimensions (Table 2), make for orbits with few enough positions that the gradations in the discrepancy values become clear.

Sequences in any $R_{h_{\min }}$ with $V(S)$ that better the bound of (7.10) can be generated by using some minimum search algorithm, for example by selecting the smallest discrepancy going forward at any graph vertex where there is a choice.

It's worth noting that a disadvantage to the multi-symbol technique is that for larger $k$ values, and larger maximum $d_{i}$ values, the number of cells for the associated simplices can grow quite large, at a geometric rate. The paring step will produce a large reduction in the number of cells to track, what becomes the total dimension of the $S_{\alpha}$ matrices. A further reduction, or really partitioning, will occur when the closed routes are traced, producing often more than 1 block rows. The matrices dealt with at all points are very sparse, and may be more efficiently handled by being treated as such; they also should be more manageable after the initial reduction and route-finding steps are completed. Nonetheless, the condensed block rows can be sizeable, even at $k=4$ and $\max d_{i}=8$, as shown in Table 2 .

7.7. Regularity matrices for longer symbol alphabets. To construct regularity matrices for splice-only, pad-only, and pad-and-splice schemes for arbitrary symbol alphabet $\mathcal{A}$ under symbol probabilities $p_{i}$, the corresponding $S_{\alpha}$ matrix set is generated first, as above. The appendix contains a suggested algorithm for efficient derivation. Since a more precise estimate of the minimal simplex height other than what (7.11) offers is beyond the scope of the paper, in practice the minimal 


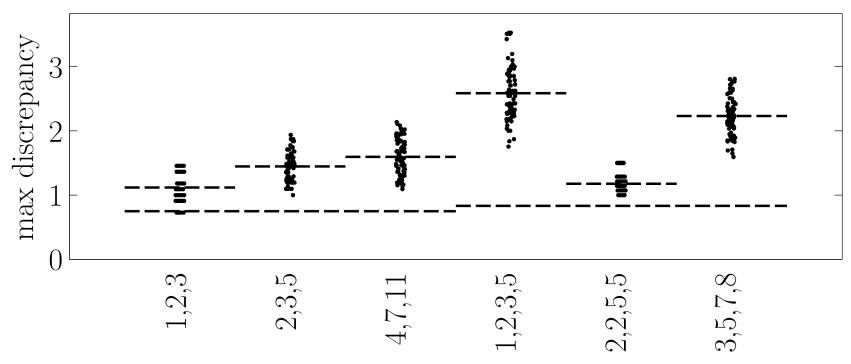

FigURE 15. scatterplot of the maximum discrepancies of $75 S_{\alpha}$ product chains of length 1 e 4 chosen uniformly from an $R_{h_{\min }}$ corresponding to the indicated $k=3$ - or 4-symbol shift sets, $d_{1}, \ldots, d_{k}$ (horizontal jitter added for clarity); the lower dashed lines are the optimal bounds on the lowest discrepancy sequences, $1-1 /(2(k-1))$; the upper dashed lines are the averages

height $h_{\text {min }}$ may be found by running the algorithm repeatedly at different simplex heights, until the minimum is found that produces non-null matrices at the end of the reduction stage. Once the $S_{\alpha}$ are obtained, the procedure is straightforward. For splice-only, we've

$$
M_{i}=p_{i} \delta \mathbb{I}+(1-\delta) S_{i} .
$$

For pad-only as well as pad-and-splice,

$$
P=\mathbb{I}+\delta \sum_{i} p_{i} S_{i}+\delta^{2}\left(\sum_{i} p_{i} S_{i}\right)^{2}+\ldots=\frac{1}{\mathbb{I}-\delta \sum_{i} p_{i} S_{i}} .
$$

The proof of convergence of the geometric series is similar to that of Claim 4. We decompose the $S_{\alpha}$, if need be, into block diagonals, each block row comprising a strongly connected graph. Irreducibility of the $\sum_{i} S_{i}$ matrix components in a given block row follows from the irreducibility of the adjacency matrix, as in Section 7.3.

Perron-Frobenius can then be applied as in Claim 4, showing that padding matrix $P$ has blockwise top eigenvalues $\lambda_{j}<1$ for all $\delta \in[0,1]$. The norm of the geometric series's $k$ th term may then be bounded by $c(1-\epsilon)^{k}$ where $\epsilon$ is matched to the largest $\lambda_{j}$. This follows from noting the sum of the entrywise norms of all $l$ blocks is bounded asymptotically in $k$ by some constant times $l(1-\epsilon)^{k}$, and using the equivalence of norms.

The three main sequence types may then be formed as before,

$$
Q V_{i} Q \ldots Q V_{i} Q
$$

where for splice-only, $Q=\mathbb{I}, V_{i}=M_{i}$; for pad-only, $Q=P, V_{i}=S_{i}$; and for pad-and-splice, $Q=P, V_{i}=M_{i}$. For a true probability distribution, the entrywise norms must be normalized, as in the binary case, by the usual factor,

$$
c_{N}=\frac{1}{\left\|\left(Q\left(\sum_{i} V_{i}\right)\right)^{m} Q\right\|_{e n}} .
$$

The norms, normalized or not (and their logarithms), may also always be compared to those of other multi-symbol sequences of the same length, to determine relative regularity. 
7.8. The central limit theorem and z-scores for longer symbol alphabets. In the interest of assigning z-scores, the central limit theorem can be applied in the case $|\mathcal{A}|>2$ as well. If after the reduction and route finding steps the $S_{\alpha}$ contain more than one block row, there are some additional considerations.

Let $R_{1}, \ldots, R_{k}$ denote the regularity matrix product pairs of interest: $R_{i}=Q V_{i}$, constructed as above. Let $\left\{D_{i, 1}, \ldots, D_{i, k}\right\}$ be the set of $n_{i}$-dimensional matrices corresponding to the $i$ th block row of the $\left\{R_{l}\right\}_{l}$ set, $i \in\left[1, i_{\max }\right]$. Let $\mu$ be the overall discrete matrix probability measure, and note that the probability $P\left(D_{i, j}\right)$ is equal for each $i$, with $j$ fixed. We are interested in random matrix products between members of the set,

$$
\left[\begin{array}{cccc}
D_{1,1} & 0 & 0 & \cdots \\
0 & D_{2,1} & 0 & \cdots \\
\vdots & 0 & \cdots & D_{i_{\max }, 1}
\end{array}\right], \ldots,\left[\begin{array}{cccc}
D_{1, k} & 0 & 0 & \cdots \\
0 & D_{2, k} & 0 & \cdots \\
\vdots & 0 & \cdots & D_{i_{\max }, k}
\end{array}\right]
$$

That each block row obeys the central limit theorem follows easily from Theorem 2 , and the joint irreducibility of the $S_{i}$ components within the $\left\{D_{i, j}\right\}_{j}$ matrix sets, as in the binary case.

If the $S_{\alpha}$ matrices after the reduction and route finding steps contain only one block row $\left(i_{\max }=1\right)$, the procedure for finding the z-score is straightforward. We apply the $\log$ of the norm of the product to the CLT-derived normal distribution, as before.

To treat the case $i_{\max }>1$, we'll first partition the block rows into equivalence classes under symbol permutations.

Given block rows $a$ and $b$, define equivalence relation $E$ as $a \sim b$ iff there is some symbol permutation, $\sigma$, and some permutation matrix similarity transformation, $\Phi$, such that $\Phi^{-1} D_{a, j} \Phi=D_{b, \sigma(j)}$ for all symbols $j \in[1, k]$. All block rows equal under $E$ are identical under some pairwise reordering of their symbols, up to matrix $\Phi$-similarity. Equivalence relation $E$ then partitions the block rows of the $S_{\alpha}$, and therefore the $R_{i}$, into $n_{e c} \geq 1$ equivalence classes. This also sorts the respective block row graphs into isomorphism groupings, where the isomorphisms are the result of edge symbol permutations. (We note that graphs code all and only the surviving $S_{i}$ matrix product sequences; if graphs $G_{i_{1}}$ and $G_{i_{2}}$, corresponding to block rows $i_{1}$ and $i_{2}$, are isomorphic, then there is a bijection between the surviving products of matrix set $i_{1}$ to those of matrix set $i_{2}$, after the appropriate symbol permutation.)

Let $U_{t}$ denote the set of block row indices in the $R_{i}$ that form the equivalence class indexed by $t$. Then $\left\{D_{U_{t}(j), 1}, \ldots, D_{U_{t}(j), k}\right\}$ denotes the set of matrices corresponding to the $j$ th block row in equivalence class $t$, of size $s_{e q}$.

As an example, consider the $k=4$ symbol, equiprobable case. The template sequences associated with the $S_{\alpha}$ matrices will amount to $\overline{1234}$ and its permutations between circular shift equivalence classes: $\overline{1243}, \overline{1324}, \overline{1342}, \overline{1423}$, and $\overline{1432}$ (or any circular shift, $1234 \rightarrow 4123$ etc., of each). The four $S_{\alpha}$ template matrices will consist of six block rows, all in the same equivalence class. So $n_{e c}=1$, and $U_{1}=$ $\{1,2,3,4,5,6\}$. The respective block row graphs (e.g. simply $1 \rightarrow 2 \rightarrow 3 \rightarrow 4$, with one more directed edge from the last element to the first), will be isomorphic under the appropriate symbol permutation. In fact, all permutations of the symbol set $\{1,2,3,4\}$ are covered-each block row graph automorphic under circular shifts, with the inter-block-row isomorphisms linked with the remaining symbol permutations. 
We first claim the block rows in an equivalence class all converge to the same $\mathcal{N}\left(m \lambda, m \sigma^{2}\right)$ distribution. This relies on the fact that matrix position permutations (up to $\Phi$ similarity) within any $U_{t}$ are only permissible within sets of symbols with equivalent probabilities.

Consider the block rows of equivalence class $U_{t}$. Let $A_{i}$ denote the matrix in the $i$ th column of some block row $a$; under our conventions, this is the matrix in block row $a$ assigned to symbol $i$. Define the range of $A_{i}$ within $U_{t}, R\left(A_{i}\right)$, to be all block row columns containing $\Phi^{-1} A_{i} \Phi$, under some appropriate permutation matrix transformation.

Lemma 3. All symbols within set $R\left(A_{i}\right)$ must have probability $p_{i}$.

Proof. We have that $P\left(A_{i}\right)=p_{i}$ by the original probability-symbol assignments that went into constructing the $S_{\alpha}$. Any symbol in $R\left(A_{i}\right) \backslash\{i\}$ must have probability $p_{R\left(A_{i}\right)}$ for the same reasons.

Consider a block row, call it $b$, for which $\Phi^{-1} A_{i} \Phi$ is in column (symbol position) $\xi \in R\left(A_{i}\right)$, where permutation matrix $\Phi$ maps matrices from block row $a$ to $b$ (under some permutation on symbols, $\sigma$ ).

By construction of the $S_{\alpha}$, the proportion of matrix $A_{i}$ in surviving sequences in block row a must be $p_{i}$. So too must the proportion of $\Phi^{-1} A_{i} \Phi$ under products of the set $\left\{\Phi^{-1} A_{1} \Phi, \ldots, \Phi^{-1} A_{k} \Phi\right\}$. We conclude $p_{\xi}$ must equal $p_{i}$. The same argument applies to all other block rows that gave rise to $R\left(A_{i}\right)$, completing the proof.

Note that overlapping ranges between different $A_{i}$, by extension all share the same $p_{i}$.

Lemma 4. The random matrix probability measures specific to the block rows within a given $U_{t}$ are the same, up to a permutation matrix similarity transform.

Proof. Let permutation matrix similarity transform $P$ map matrices from block row $a$ to $b$. Then probability measure $\mu_{b}$ has support in terms of block row $a$ 's matrices: $\left\{\Phi^{-1} A_{1} \Phi, \ldots, \Phi^{-1} A_{k} \Phi\right\}$, where the probability weighting for each $A_{i}$ is the same as for $\Phi^{-1} A_{i} \Phi$, by Lemma 3. With $a, b$ arbitrary, this shows the measures are all the same, up to some set of pairwise similarity transforms.

By the Lemmas, since each block row in the equivalence class has the same probability measure up to a $\Phi$ similarity transform, which does not affect entrywise norms, by the central limit theorem each block row converges weakly to the same $\mathcal{N}\left(m \lambda, m \sigma^{2}\right)$ distribution.

Next we'll show one block row equivalence class will eventually dominate in distribution over all the others.

Lemma 5. The Lyapunov exponent for equivalence class $U_{t}$ is equal to the Lyapunov exponent for any block row in $U_{t}$.

Proof. Let $Y_{l}$ denote the random matrix variable associated with the sub-matrix consisting of only the block rows in equivalence class $U_{t}$. Define block row matrix product $\Pi_{j}\left(i_{m}, \ldots, i_{1}\right)=D_{U_{t}(j), i_{m}} \cdots D_{U_{t}(j), i_{1}}$ for symbol sequence $i_{m}, \ldots, i_{1}$, $j \in\left[1, s_{e q}\right]$. Let $X_{l}$ be a random variable with the usual generalized Bernoulli distribution, $P(s)=p_{s}$ for each symbol $s \in[1, k]$.

The distribution of interest can be expressed as,

$$
\log \left\|Y_{m} \cdots Y_{1}\right\|=\log \left(\left\|\Pi_{1}\left(i_{m}, \ldots, i_{1}\right)\right\|_{e n}+\ldots+\left\|\Pi_{s_{e q}}\left(i_{m}, \ldots, i_{1}\right)\right\|_{e n}\right)_{i_{l} \in X_{l}}
$$


Define bijection, $f_{\sigma}$, between norms:

$$
f_{\sigma}\left(\left\|D_{U_{t}(j), i_{m}} \cdots D_{U_{t}(j), i_{1}}\right\|_{e n}\right)=\left\|D_{U_{t}(j), \sigma\left(i_{m}\right)} \cdots D_{U_{t}(j), \sigma\left(i_{1}\right)}\right\|_{e n}
$$

i.e. the result on the matrix product of applying the action of some symbol permutation $\sigma$ on symbol sequence $i_{m}, \ldots, i_{1}$.

Then distribution 7.15 can be written as,

$\log \left(\left\|\Pi_{1}\left(i_{m}, \ldots, i_{1}\right)\right\|+f_{\sigma_{1}}\left(\left\|\Pi_{1}\left(i_{m}, \ldots, i_{1}\right)\right\|\right)+\ldots+f_{\sigma_{s_{e q}-1}}\left(\left\|\Pi_{1}\left(i_{m}, \ldots, i_{1}\right)\right\|\right)\right)_{i_{l} \in X_{l}}$,

where the $\sigma_{i}$ permute between the original symbol ordering of $\Pi_{1}$ and the permutations of block rows $i+1$.

By Theorem 1 and Lemma 4, any $U_{t}$ block row random product obeys,

$$
\lim _{m \rightarrow \infty} \frac{1}{m} \log \left\|Z_{m} \cdots Z_{1}\right\|=\lambda,
$$

with probability 1 ; or, equivalently,

$$
\log \left\|Z_{m} \cdots Z_{1}\right\|=m \lambda+\mathcal{O}\left(m^{1-\epsilon}\right) \Leftrightarrow\left\|Z_{m} \cdots Z_{1}\right\|=e^{m \lambda} e^{\mathcal{O}\left(m^{1-\epsilon}\right)},
$$

a.s.

From 7.16 we have,

$$
\log \left\|Y_{m} \cdots Y_{1}\right\|=\log \left(e^{m \lambda} e^{\mathcal{O}\left(m^{1-\epsilon}\right)}+\ldots+e^{m \lambda} e^{\mathcal{O}\left(m^{1-\epsilon}\right)}\right)
$$

since each bijection is fixed, and (7.17) applies almost surely to any random product. This produces, as desired,

$$
\lim _{m \rightarrow \infty} \frac{1}{m} \log \left\|Y_{m} \cdots Y_{1}\right\|=\lambda .
$$

Let $\left\{\lambda_{U_{1}}, \ldots, \lambda_{U_{f}}\right\}$ be the Lyapunov exponents corresponding to the set of equivalence classes, 1 through $n_{e c}$. By Lemma 5 and its proof, random matrix products of the $R_{i}$ will obey,

$$
\begin{aligned}
& \log \left\|S_{m} \cdots S_{1}\right\|=\log \left(e^{m \lambda_{U_{1}}} e^{\mathcal{O}\left(m^{1-\epsilon}\right)}+\ldots+e^{m \lambda_{U_{f}}} e^{\mathcal{O}\left(m^{1-\epsilon}\right)}\right) \\
& =\log e^{m \lambda_{\alpha}} e^{\mathcal{O}\left(m^{1-\epsilon}\right)}\left(1+\mathcal{O}\left(e^{m\left(\lambda_{\beta}-\lambda_{\alpha}\right)}\right) \approx m \lambda_{\alpha}+\mathcal{O}\left(m^{1-\epsilon}\right),\right.
\end{aligned}
$$

where we've assigned $\lambda_{\alpha}$ and $\lambda_{\beta}$ to the equivalence classes with the largest and next-to-largest Lyapunov exponents, respectively. We conclude that provided $m$ is large enough, the block rows with the largest Lyapunov exponent, $\lambda_{\alpha}$, will dominate the norm. (With the additional assumption the two largest Lyapunov exponents from equivalence classes 1 through $n_{e c}$ are not exactly equal; treatment of such a case is beyond the scope of this paper.)

Let $U_{t^{\prime}}$ denote the equivalence class corresponding to the largest Lyapunov exponent. In the interest of forming meaningful z-scores, the distribution of the sum over block rows of the $R_{i}$ in $U_{t^{\prime}}$ when $\left|U_{t^{\prime}}\right|>1$ needs to be considered.

In general, if we have a set of block diagonal matrices, where every block row has the same Lyapunov exponent and obeys an identical normal distribution, we'd like to know the distribution of their sum.

Choose any two block rows in $U_{t}$. From the result of Lemma 4, the logs of the norms follow the same normal distribution, so the norms themselves follow the same lognormal distribution, $h$. 
The sums of their norms as a random variable, from 7.16, may be written,

$$
\left(\left\|\Pi_{1}\left(i_{m}, \ldots, i_{1}\right)\right\|+f_{\sigma}\left(\left\|\Pi_{1}\left(i_{m}, \ldots, i_{1}\right)\right\|\right)\right)_{i_{j} \in X_{j}} .
$$

This amounts to forming a new distribution via a composition-convolution of $h$ : $h *\left(h \circ f_{\sigma}\right)$. In general, the sum of lognormal distributions is not lognormal. And in this case, depending on the bijection $f_{\sigma}$, the resulting distribution, a sum of the lognormal probability mass and the pdf under some reordering of the sequence domain determined by $f_{\sigma}$, need not be lognormal either, making the result back under log scaling not necessarily normal.

In spite of this drawback, we can still assign meaning to the log of the norm of a matrix product over all block rows in an equivalence class: it approximately assigns the maximal regularity score over all regularity templates in the equivalence class.

To show this, given sequence $s$ the action of the various block rows in some $U_{t}$ amounts to computing a regularity score with the same set of matrices under each symbol permutation in the equivalence class. In the example of $k=4$ symbols with equiprobable weighting, sequence $s=124312243124312$ is very regular under $\overline{1243}$, but not as regular under, for example, $\overline{1432}$; each block row scores the sequence against a different template permutation ( 6 total).

Denote by $r_{i}(s)$ the symbols in sequence $s$ rendered as a matrix product by the matrices in block row $U_{t}(i)$. Then the block row sum associated with $s$ can be written as,

$$
y=\log \left(\left\|r_{1}(s)\right\|_{e n}+\left\|r_{2}(s)\right\|_{e n}+\ldots+\left\|r_{s_{e q}}(s)\right\|_{e n}\right) .
$$

Denoting the maximal value of $\left\|r_{i}(s)\right\|_{e n}$ over all $i$ by $\left\|r^{\prime}(s)\right\|_{e n}$, we have,

$$
\log \left(\left\|r^{\prime}(s)\right\|_{e n}\right)<y \leq \log \left(s_{e q}\right)+\log \left(\left\|r^{\prime}(s)\right\|_{e n}\right) .
$$

We can interpret the quantity $y$ as selecting the norm of the block row corresponding to the best regularity template match to $s$, and taking its logarithm. The relative effect of the $\log \left(s_{e q}\right)$ term vanishes as $m \rightarrow \infty$.

7.9. Procedure for calculating z-scores. The section concludes with the recommended procedure for calculating z-scores.

Compute the log of the norm for sequence $s$ as usual, creating the matrix product from the full $R_{i}$ matrix set over all symbols in $s$ (that the $R_{i}$ are likely block diagonal offers a savings in computational resources). The z-score is computed from the $\mathcal{N}\left(m \lambda, m \sigma^{2}\right)$ distribution associated with any block row in the equivalence class with the largest Lyapunov exponent. While the distribution over random products of the $R_{i}$ isn't necessarily normal, we may think of the z-score of sequence $s$ as comparing, approximately, the maximum of the maximally regular value for $s$ over all equivalence classes, against the limiting distribution for any of the $\lambda$-maximal block rows, as $m \rightarrow \infty$.

It's worth emphasizing that though the block row(s) having the maximal Lyapunov exponent do eventually dominate the distribution, it is possible for sequence $s$ to be very regular under the templates of some other block row equivalence class (see Section 4.1, on the potential for large outliers). It is not enough to only compute the matrix product under block rows in the maximal $\lambda$ equivalence class. This risks missing sequences with high regularity under block row equivalence classes with lower $\lambda$ 's.

Given the foregoing, to summarize the procedure for assigning a z-score to $S_{m} \cdots S_{1}$ random products of block diagonal matrices, 
- Estimate the Lyapunov exponent for each block row equivalence class, indexed by $U_{t}$; if the symbolic sequences under test are expected to have each symbol in approximate proportion $p_{i}$ (as is usually the case), the Monte Carlo routines are run under an extension of the binary alphabet's $p$-proportional distribution, (4.2): $\mu_{p}=p_{1} \delta_{D_{i, 1}}+\ldots+p_{k} \delta_{D_{i, k}}$

- For test sequence $s$, take the associated $R_{i}$ matrix product over all block rows, and compute the z-score by applying $\log \left\|S_{m} \cdots S_{1}\right\|$ to the $\mathcal{N}\left(m \lambda, m \sigma^{2}\right)$ distribution corresponding to the maximal equivalence class Lyapunov exponent ${ }^{\dagger}$

( ${ }^{\dagger}$ Note that the matrix product length, $m$, should be large enough that the block rows indexed by the maximal $U_{t^{\prime}}$ dominate the norm of the $R_{i}$ products; this depends on the $e^{m\left(\lambda_{\beta}-\lambda_{\alpha}\right)}$ factor associated with the largest two Lyapunov exponents; see (7.18).)

\section{Distribution entropy}

For any given alphabet, $\mathcal{A}$, and shift set $\left\{d_{1}, \ldots, d_{k}\right\}$, let $\rho$ be the proportion spread parameter: for binary alphabets $\rho$ is the matrix dimension, while for longer alphabets it's the simplex height. We may then parametrize any regularity distribution by $\left\{\left\{p_{1}, \ldots, p_{k}\right\}, \delta, \rho\right\}$, where the probabilities' relation to shift values are given by (7.1). For each sequence length $m$, there is an associated random vector, $\mathcal{X}_{m}=\left(X_{1}, \ldots, X_{m}\right)$, with random variables $X_{i}$, each with state space $\mathcal{A}$. The probability of a given sequence, $P\left(X_{1}=x_{1}, \ldots, X_{m}=x_{m}\right)$, is given by the usual normalized matrix product.

The entropy of the random vectors $\mathcal{X}_{m}$ is, (8.1)

$$
H\left(\mathcal{X}_{m}\right)=-\sum_{i_{1}, \ldots, i_{m}=1, \ldots, k} c_{N}\left\|Q V_{i_{m}} \cdots Q V_{i_{1}} Q\right\|_{e n} \log \left(c_{N}\left\|Q V_{i_{m}} \cdots Q V_{i_{1}} Q\right\|_{e n}\right) .
$$

In the interest of a stable average information content per symbol, we can ask if the sequence of random vectors, $\mathcal{X}_{1}, \mathcal{X}_{2}, \ldots$, has a well-defined entropy rate. That is, does the limit,

$$
h_{r}=\lim _{m \rightarrow \infty} \frac{H\left(\mathcal{X}_{m}\right)}{m},
$$

exist. We show that it does. In the case the $\left\{Q, V_{1}, \ldots, V_{k}\right\}$ are composed of a single block row, we can associate the $\mathcal{X}_{m}$ with a stationary stochastic process. We further show the process is mixing. As a consequence, by the Shannon-McMillan-Breiman theorem, the asymptotic equipartition property (AEP) holds. The reasoning is extended to matrices with more than one block row, leading to a robust definition of symbolic sequence homogeneity entropy. The section concludes with a Monte Carlo resampling method for numerically estimating the entropy. First we'll take a look at the entropy at the endpoints, $\delta=\{0,1\}$.

8.1. Entropy at the $\delta=0, \delta=1$ extremes; topological entropy on shifts. At the $\delta=1$ extreme, recall by construction of the padding matrices, $Q$, and the symbol matrices $V_{i}$, the distribution in the splice-only, and pad-and-splice cases collapsed to a pure generalized Bernoulli distribution: $P\left(\alpha_{i}\right)=p_{i}$, i.i.d. The entropy, and a well-defined entropy rate in these cases follows immediately. In the case of pad-only at $\delta=1$, the situation is not so reducible, and we must treat it alongside the cases $\delta \in(0,1)$. 
At the $\delta=0$ extreme, $Q=\mathbb{I}$ and $V_{i}=S_{i}$ in all cases. This leaves us with

$$
H\left(\mathcal{X}_{m}\right)=-\sum_{i_{1}, \ldots, i_{m}=1, \ldots, k} c_{N}\left\|S_{i_{m}} \cdots S_{i_{1}}\right\|_{e n} \log \left(c_{N}\left\|S_{i_{m}} \cdots S_{i_{1}}\right\|_{e n}\right),
$$

with $c_{N}=1 /\left(\sum_{i} S_{i}\right)^{m}$. This is related to the topological entropy for symbolic dynamical systems, as we'll show.

Let $(Y, \sigma)$ denote a shift space, i.e. a set $Y$ of two- (or one-) sided infinite sequences in $\mathcal{A}^{\mathbb{Z}}$, with $\sigma$ the shift operator,

$$
(\sigma(y))_{j}=y_{j+1},
$$

$\forall y \in Y$ (see for instance [32]). A subshift is a shift space where $Y$ is closed under the shift map. Subshifts may be defined by a possibly infinite set of forbidden blocks (contiguous substrings), $\mathcal{F}$, where no sequence in $Y$ contains any forbidden block of $\mathcal{F}$. If the cardinality of $\mathcal{F}$ is finite, the shift is termed a subshift of finite type (SFT). Sofic shifts consist of those subshifts that are 'factors' (images of onto homomorphisms) of SFTs. All SFTs are sofic, but not all sofic shifts are SFTs.

Consider a directed graph with edges labeled from alphabet $\mathcal{A}$. In the context of shifts, the set of all bi-infinite walks on the edges of such a graph is termed an edge shift. Every sofic shift may be represented by an edge shift, and vice-versa.

The template sequences formed from $\lim _{m \rightarrow \infty} \prod_{j=1, \ldots, m} S_{i_{j}}$ may be regarded as a set of (bi-)infinite subshifts, one for each block row: since each block row was representable by an edge-labeled directed graph (see Section 7.3), the corresponding sequence sets are at least sofic shifts. Since every finite union of subshifts is also a subshift, the template sequences in aggregate, over all block rows, are also a subshift. We also note the template shifts may be SFTs, as in the simplest example, $p=1 / 2$, with matrix dimension $s=2(\mathcal{F}=\{00,11\})$, or strictly sofic, as with $p=1 / 2, s=3$ (where sequences of type $11(01)^{j} 10, j>=0$, are forbidden, but sequences of type $11(01)^{j} 00, j \geq 0$, are allowed; $\mathcal{F}$ as a consequence must have infinite cardinality).

Let $Y$ be a shift space. Let $B_{m}$ denote the number of allowed blocks of length $m$. Then the topological entropy for $Y$ (see for instance [25]) is defined as,

$$
h(Y)=\lim _{m \rightarrow \infty} \frac{1}{m} \log \left(B_{m}\right) .
$$

We'll see this relates to $H\left(\mathcal{X}_{m}\right) / m, m>>1$, at the $\delta=0$ limit, through a decomposition on walks within their bounding simplex, what was touched on briefly in the binary alphabet case, Section 6 .

Recall by construction from the geometry of the shifts of unit shear cells within the bounding simplex, each 1 in the $m$-fold product, $\prod_{1, \ldots, m} S_{i_{j}}$, corresponded to exactly one surviving 'shift route,' provided it was a prefix to an infinite surviving sequence. The entrywise norm $\left\|\prod_{1, \ldots, m} S_{i_{j}}\right\|_{\text {en }}$ then corresponded to the number of such surviving shift routes, each physically parallel to any other.

By extension, the number of allowed sequences, or blocks, of length $m$ were given by

$$
B_{m}=\left\|\left(\sum_{1, \ldots, k} S_{i}\right)^{m}\right\|_{e n}=\frac{1}{c_{N}(m)},
$$

where $c_{N}(m)$ was the normalization constant (the $m$ to emphasize its dependence on sequence length). Since the matrix $A=\sum_{1, \ldots, k} S_{i}$ is primitive, we have by 
Perron-Frobenius,

$$
\log \left(B_{m}\right)=-\log \left(c_{N}(m)\right) \rightarrow m \lambda
$$

where $\lambda$ is the spectral radius of $A$. Consulting (8.3), the topological entropy is therefore the spectral radius.

Note that if all, or an asymptotic majority of walks have multiplicity one, then by (8.2) the entropy converges to,

$$
H\left(\mathcal{X}_{m}\right) \rightarrow-\sum_{\text {all walks }} c_{N} \log \left(c_{N}\right)=-\log \left(c_{N}\right),
$$

since $c_{N}$ is just the reciprocal of the total number of allowed walks in the $\delta=0$ case. This is the basis for the following theorem, linking the distribution entropy $H\left(\mathcal{X}_{m}\right)$ at $\delta=0$ to the topological entropy.

Theorem 4. Given regularity parameter set $\left\{\left\{p_{1}, \ldots, p_{k}\right\}, \delta, \rho\right\}$, with $\delta=0$, and bounding simplex height at its minimum, $\rho=h_{\min }$, the entropy rate, $h_{r}=$ $\lim _{m \rightarrow \infty} H\left(\mathcal{X}_{m}\right) / m$, is well defined and equal to the topological entropy of the shift associated with the sequences permitted under the $S_{\alpha}$ template set. For binary alphabets, the equality also holds for all allowed 1-dimensional simplex heights, $\rho \geq d_{0}+d_{1}$.

Proof. First we develop the decomposition on allowed walks. Let a 'full range' walk, $W_{u}$, in a simplex of height $h=u /(k-1)$ be an infinite walk that has touched each of the $k$ boundary faces of the bounding simplex at least once. For all such walks, for any block within it, there is a uniform bound, $M$, on the number of steps required for it to contact all $k$ faces of the bounding simplex. At most, $M$ is equal to the total number of unit shear cells in the bounding simplex. It follows that prefixes of a full range walk, $W_{u}(m)$, eventually have multiplicity 1 for all $m>M$, since there can be no walks parallel to it (shift the whole walk by any amount, $d_{i}$, and some portion must lie outside the bounding simplex).

We can decompose the total set of infinite-surviving walks within a bounding simplex of altitude $u /(k-1)$ by those of full range in the simplex of $u$ height units, $W_{u}$, plus those of full range at $u-1$ units, $W_{u-1}$, and so on, down to the minimal simplex height, $u_{\min }=(k-1) h_{\min }$. (From here on, we'll generally use height units, $u$, to refer to simplex heights, keeping in mind the actual height was defined as $h=u /(k-1)$ in Section 7.1.)

Any infinite walk in a simplex of height $u$ must be a full range walk within some interior simplex of height $u-j, j \geq 0$. From the formula in Section 7.2 and a little geometry, there are exactly $\left(\begin{array}{c}j+k-1 \\ k-1\end{array}\right)$ simplices of dimension $(k-1)$ and of height $u-j$ within a bounding simplex of height $u$.

We have the decomposition,

$$
\begin{aligned}
B_{m} & =\left|W_{u}(m)\right|+\left(\begin{array}{c}
k \\
k-1
\end{array}\right)\left|W_{u-1}(m)\right|+\left(\begin{array}{c}
k+1 \\
k-1
\end{array}\right)\left|W_{u-2}(m)\right|+\ldots \\
& +\left(\begin{array}{c}
k+u-u_{\min }-1 \\
k-1
\end{array}\right)\left|W_{u_{\min }}(m)\right| .
\end{aligned}
$$


The $\left|W_{u-j}(m)\right|$ may be derived from $\sum S_{i}$ powers recursively as follows. Denote by $S_{i}^{(u)}$ an $S_{i}$ derived from a simplex of $u$ height units. Then,

$$
\left|W_{u_{\min }}(m)\right|=\left\|\left(\sum_{1, \ldots, k} S_{i}^{\left(u_{\min }\right)}\right)^{m}\right\|_{e n}
$$

and for the recursion,

$$
\begin{aligned}
\left|W_{u_{\min }+j}\right| & =\left\|\left(\sum_{1, \ldots, k} S_{i}^{\left(u_{\min }+j\right)}\right)^{m}\right\| \|_{e n}-\left(\begin{array}{c}
k \\
k-1
\end{array}\right)\left|W_{u_{\min }+j-1}(m)\right|-\ldots \\
& -\left(\begin{array}{c}
k+j-1 \\
k-1
\end{array}\right)\left|W_{u_{\min }}(m)\right| .
\end{aligned}
$$

Already we see that $H\left(\mathcal{X}_{m}\right)$ at $\delta=0$ is related to the topological entropy through (8.2), and through (8.5), (8.6), and (8.7), which are fundamentally a decomposition based on word frequencies at various simplex heights, expressed through powers of the $S_{i}$ sums. The decomposition (8.5) also offers an alternate way to compute the entropy at $\delta=0$, what requires computing $A_{u-j}=\sum_{i} S_{i}^{(u-j)}$ for all $j \in$ $\left[0, u-u_{\min }\right]$.

We now prove the result for simplices of minimal height. Suppose we start with a bounding simplex with $u=u_{\text {min }}$. Then all walks of length $m>M$ have multiplicity one. So each product, $\prod_{1, \ldots, m} S_{i_{j}}$, is either 0 or 1 . Then by the formulation for entropy at the $\delta=0$ extreme, (8.2), and (8.5),

$$
H\left(\mathcal{X}_{m}\right)=-\left\|\left(\sum_{1, \ldots, k} S_{i}\right)^{m}\right\|_{e n} c_{N}(m) \log \left(c_{N}(m)\right)=\log \left(1 / c_{N}(m)\right),
$$

for $m>M$. The entropy rate, $h_{r}$, then is well-defined, and equal to the topological entropy, by (8.4).

More generally, it is easy to show in the binary alphabet case that the number of full range walks of span $u,\left|W_{u}\right|$, increase at a geometrically faster rate than those of spans $u-1, u-2$, and so on. This makes $B_{m} /\left|W_{u}\right| \rightarrow 1$ as $m \rightarrow \infty$. By the same reasoning as in the $u=u_{\text {min }}$ case (where the full range walks of span $u$, each with multiplicity 1 , also dominate), this would also make $H\left(\mathcal{X}_{m}\right) / m$ equal to the topological entropy in the limit $m \rightarrow \infty$.

Recall from Section 6 , the general $\left\{S_{0}, S_{1}\right\}$ form in the binary case are simply $s$ by $s$ binary matrices with a diagonal of 1 's at respectively the $+d_{0}$ and $-d_{1}$ diagonal positions relative to the main diagonal. By (8.5) and (8.4), for fixed $\left\{d_{0}, d_{1}\right\}$ we are comparing $\left(\sum_{0,1} S_{i}\right)^{m}$ at $S_{i}$ dimensions $s, s-1, \ldots, d_{0}+d_{1}$.

As before, denoting $S_{i}^{(s)}$ for an $S_{i}$ of dimension $s$, consider $A_{s}=\sum_{0,1} S_{i}^{(s)}$, $A_{s-1}=\sum_{0,1} S_{i}^{(s-1)}$, and an augmented $A_{s-1}, A_{s-1}^{\prime}$, constructed by padding with 
an additional $s$ th row, and $s$ th column, with an $\epsilon \in(0,1)$ at $\left(s, s-d_{1}\right)$ and $\left(s-d_{0}, s\right)$ :

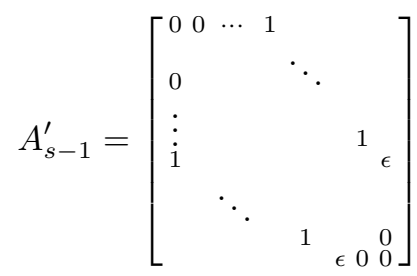

Then since $A_{s-1}^{\prime}$ is primitive, as well as $A_{s}$, and we have $A_{s-1}^{\prime} \leq A_{s}$ (entrywise) with $A_{s-1}^{\prime} \neq A_{s}$ (in total), by Perron Frobenius the spectral radius of $A_{s-1}^{\prime}$ is strictly less than that of $A_{s}$. Further, since $\left\|\left(A_{s-1}\right)^{m}\right\| \leq\left\|\left(A_{s}\right)^{m}\right\|$ by nonnegativity of the matrices, if we generalize over all $s-j$ we have the strict nesting inequalities for the spectral radii, $\rho\left(A_{s}\right)>\rho\left(A_{s-1}\right)>\ldots>\rho\left(A_{d_{0}+d_{1}}\right)$. This proves the desired dominance for (8.5) in $m, B_{m} /\left|W_{u}\right| \rightarrow 1$, showing the entropy rate is equal to the topological entropy for all binary alphabet cases.

It seems likely the number of full range walks at height $u$ would geometrically dominate the number of walks at lower heights in the general alphabet case, $k \geq 2$ as well. Though we cannot offer proof at this time, it would make the equality between topological entropy and the random vector entropy rate at $\delta=0$ valid for all regularity distributions.

8.2. Entropy of homogeneity: stationary stochastic processes, entropy rate, mixing, and the AEP. We now consider entropy in the general $\delta \in(0,1)$ case. For the random vector $\mathcal{X}_{m}$, we note the joint probabilities of sequences at the ends (e.g., for the last $l$ symbols, $P\left(X_{m-l+1}=x_{1}, \ldots, X_{m}=x_{l}\right)$ ) are prone to cutoff distortions from the regularity metric. For example in the binary case, $p=4 / 5$, matrix dimension equal to 5 , pad-and-splice, consider any sequence close to fully regular, such as $100001000 \ldots 0$. There are more opportunities at the end points to pad the sequence to fully regular: at the left end, for example, we can pad to $010 \ldots 0,0010 \ldots 0$, etc., while in the middle, on average, to pad the sequence to fully regular it would require the insertion of a complete 10000 substring.

This suggests, that for sufficiently long sequences, the middle joint distributions might stabilize. In fact, we'll show the middle joint distributions approach stationarity. This will be key to proving the existence of a general entropy rate.

For the general approach of this section, we first show the existence of the entropy rate on regularity matrices with only one block row (as is certainly the case for binary alphabets). After this is accomplished, the results will be extended to matrices with more than one block row.

8.2.1. Entropy rate and the AEP; single block row case. For the single block row case, first a lemma is required, one linking the general normalized matrix product probability measure of (7.13) and (7.14) to a stationary stochastic process (recalling matrix products by convention are in the reverse of sequence order):

Lemma 6. Given matrix set $\left\{A_{1}, \ldots, A_{k}\right\}$, let $B=\sum_{i} A_{i}$. Assume $B$ is nonnegative and irreducible. By Perron-Frobenius, let $\lambda$ be the (unique) top eigenvalue, and let $v$ and $w$ be the respectively right and left eigenvectors of $B$, normalized so that $w^{\top} v=1$. Let $\mathcal{A}$ as usual denote a $k$ symbol alphabet, and consider the space 
of bi-infinite sequences over $\mathcal{A}: \Sigma=\mathcal{A}^{\mathbf{Z}}$. Let $\mu$ be a probability measure defined on cylinder sets $C_{0}\left[x_{1}, \ldots, x_{n}\right]$ by,

$$
\mu\left(C_{0}\left[x_{1}, \ldots, x_{n}\right]\right)=\frac{\left\|v w^{\top} A_{x_{n}} \cdots A_{x_{1}} v w^{\top}\right\|}{\left\|v w^{\top} B^{n} v w^{\top}\right\|} .
$$

Then there exists a unique bi-infinite stationary stochastic process with $\mu$ defining its finite distributions.

Proof. Let $x_{j}, j \in[1, l]$ be symbols from an alphabet of length $l$, with matching matrices $A_{x_{j}}$. Denote by $\left(\left(x_{j_{1}}\right)_{i},\left(x_{j_{2}}\right)_{i+1}, \ldots,\left(x_{j_{n}}\right)_{i+n-1}\right)$ an indexed finite sequence of symbols with position indices $i, i+1, \ldots, i+n-1$. Denote by $\left(A_{j_{n}}\right)_{i+n-1} \cdots\left(A_{j_{1}}\right)_{i}$ the corresponding indexed matrix product.

We note for any matrix $C$, the indexed matrix product fraction,

$$
\frac{\left\|(C)_{0}(B)_{-1} \cdots(B)_{-j}\right\|}{\left\|(B)_{0}(B)_{-1} \cdots(B)_{-j}\right\|}
$$

approaches,

$$
\frac{\left\|(C)_{0} \lambda^{j} v w^{\top}\right\|}{\left\|(B)_{0} \lambda^{j} v w^{\top}\right\|}
$$

as $j \rightarrow \infty$, and in the limit gives,

$$
\lim _{j \rightarrow \infty} \frac{\left\|(C)_{0}(B)_{-1} \cdots(B)_{-j}\right\|}{\left\|(B)_{0}(B)_{-1} \cdots(B)_{-} j\right\|}=\frac{\left\|(C)_{0} v w^{\top}\right\|}{\left\|(B)_{0} v w^{\top}\right\|} .
$$

Denoting by $*$ the open symbol, assigned the corresponding matrix $B$, the joint probability associated with cylinder set $C_{0}\left[x_{1}, \ldots, x_{n}\right]$, i.e. associated with bi-infinite sequence $\left(\ldots, *,\left(x_{1}\right)_{0}, \ldots,\left(x_{n}\right)_{n-1}, *, \ldots\right)$, becomes the limit,

$$
\begin{aligned}
\lim _{j \rightarrow \infty} \frac{\left\|(B)_{j+n-1} \cdots(B)_{n}\left(A_{x_{n}}\right)_{n-1} \cdots\left(A_{x_{1}}\right)_{0}(B)_{-1} \cdots(B)_{-j}\right\|}{\left\|(B)_{j+n-1} \cdots(B)_{n}(B)_{n-1} \cdots(B)_{0}(B)_{-1} \cdots(B)_{-j}\right\|} \\
\quad=\frac{\left\|v w^{\top}\left(A_{x_{n}}\right)_{n-1} \cdots\left(A_{x_{1}}\right)_{0} v w^{\top}\right\|}{\left\|v w^{\top}(B)_{n-1} \cdots(B)_{0} v w^{\top}\right\|} .
\end{aligned}
$$

To extend this measure to a bi-infinite stochastic process, apply the DaniellKolmogorov extension theorem. The permutation condition is obvious. For the consistency condition, we require $C_{0}\left[x_{1}, \ldots, x_{n}\right]=C_{0}\left[x_{1}, \ldots, x_{n}, *\right]$, which is the case:

$$
\frac{\left\|v w^{\top}\left(A_{x_{n}}\right)_{n-1} \cdots\left(A_{x_{1}}\right)_{0} v w^{\top}\right\|}{\left\|v w^{\top}(B)_{n-1} \cdots(B)_{0} v w^{\top}\right\|}=\frac{\left\|v w^{\top}(B)_{n} / \lambda\left(A_{x_{n}}\right)_{n-1} \cdots\left(A_{x_{1}}\right)_{0} v w^{\top}\right\|}{\left\|v w^{\top}(B)_{n} / \lambda(B)_{n-1} \cdots(B)_{0} v w^{\top}\right\|} .
$$

Similarly, to show stationarity, we see $C_{0}\left[x_{1}, \ldots, x_{n}\right]=C_{1}\left[x_{1}, \ldots, x_{n}\right]$ :

$$
\begin{aligned}
\frac{\left\|v w^{\top}\left(A_{x_{n}}\right)_{n-1} \cdots\left(A_{x_{1}}\right)_{0} v w^{\top}\right\|}{\left\|v w^{\top}(B)_{n-1} \cdots(B)_{0} v w^{\top}\right\|} & =\frac{\left\|v w^{\top}(B)_{n} / \lambda\left(A_{x_{n}}\right)_{n-1} \cdots\left(A_{x_{1}}\right)_{0} v w^{\top}\right\|}{\left\|v w^{\top}(B)_{n} / \lambda(B)_{n-1} \cdots(B)_{0} v w^{\top}\right\|} \\
& =\frac{\left\|v w^{\top}\left(A_{x_{n}}\right)_{n} \cdots\left(A_{x_{1}}\right)_{1}(B)_{0} / \lambda v w^{\top}\right\|}{\left\|v w^{\top}(B)_{n} \cdots(B)_{1}(B)_{0} / \lambda v w^{\top}\right\|} .
\end{aligned}
$$

The lemma allows another, on the entropy rate for single block row matrices:

Lemma 7. For a distribution associated with regularity parameter set, $\left\{\left\{p_{1}, \ldots, p_{k}\right\}, \delta, \rho\right\}, \delta \in(0,1)$, where the associated matrices, $\left\{Q V_{i}\right\}_{i}$, have only one block row, the limit,

$$
\lim _{m \rightarrow \infty} \frac{H\left(\mathcal{X}_{m}\right)}{m}
$$




$$
\underbrace{* \cdots \cdots \cdots * *}_{1} \underbrace{\overbrace{1}^{x_{1} x_{2} \cdots \cdots \cdots \cdots \cdots \cdots \cdots x_{n-1} x_{n}}}_{\mathrm{n}} \underbrace{* \cdots \cdots \cdots \cdots * \cdots \cdots \cdots \cdots}_{\mathrm{l}}
$$

Figure 16. Portioning an $m$-long sequence; depicted is a middle cylinder set, the middle $n$ symbols fixed, the “*”s representing an unspecified symbol. For sufficiently large $l$, the joint distribution on any of the middle $n$ symbols is stationary to arbitrary precision.

exists.

Proof. Let $n, l>0$. Divide the symbol sequence of length $m=n+2 l$ into three substrings: one of length $l$ at each end, and a substring of length $n$ in the middle. See Figure 16.

For $\delta \in(0,1)$, we'll consider the joint distributions of sequences corresponding to each of the three sections, then consider the associated entropies.

Let $\Sigma_{m}$ denote the set of all sequences of length $m$. For the middle section, we are considering the joint distribution over all finite cylinder sets of type $C_{l+1}\left[x_{1}, \ldots, x_{n}\right]=\left\{s \in \Sigma_{m} \mid s_{l+j}=x_{j}, 1 \leq j \leq n\right\}$; i.e. sequences described by $\left(*, \ldots, *, x_{1}, \ldots, x_{n}, *, \ldots, *\right)$, where $*$ represents an unspecified symbol, of which there are $l$ at each end.

The probability of any such middle cylinder set is simply,

$$
P\left(\left[X_{l+1}=x_{1}, \ldots, X_{l+n}=x_{n}\right]\right)=\frac{\left\|\left(Q \sum V_{i}\right)^{l} Q V_{x_{n}} \cdots Q V_{x_{1}}\left(Q \sum V_{i}\right)^{l} Q\right\|}{\left\|\left(Q \sum V_{i}\right)^{m} Q\right\|}
$$

where the denominator accounts for the normalization constant, and $\left(Q \sum V_{i}\right)^{l} Q$ encodes a substring of length $l$ of unspecified symbols.

We now consider the matrix products of form $\left(Q \sum V_{i}\right)^{l}$. From the developments of Sections 3, 5, 6, and 7, individual multiplicands, $Q \sum V_{i}$, when $\delta \in(0,1)$, are either strictly positive (for either of the padding cases) or primitive (for the spliceonly case). This allows the application of Perron-Frobenius:

$$
\left(Q \sum V_{i}\right)^{l} \rightarrow \lambda^{l} v w^{\top}
$$

as $l \rightarrow \infty$, using the notation from Lemma 6 .

For sufficiently large $l$, the probability of (8.8) is approximately,

$$
P\left(\left[X_{l+1}=x_{1}, \ldots, X_{l+n}=x_{n}\right]\right) \approx \frac{\left\|v w^{\top} Q V_{x_{n}} \cdots Q V_{x_{1}} v w^{\top} Q\right\|}{\left\|v w^{\top}\left(Q \sum V_{i}\right)^{n} v w^{\top} Q\right\|} \equiv P_{e s t},
$$

where we've factored out the common $\lambda^{2 l}$ from both numerator and denominator. By Lemma 6 , letting $A_{i}=Q V_{i}, P_{\text {est }}$ can be extended to a bi-infinite stationary stochastic process.

Let $\xi$ represent a matrix of equal dimensions, composed entirely of 1's. For sufficiently large $l$ the approximation introduces the entry-wise error,

$$
\left|\frac{\left(Q \sum V_{i}\right)^{l}}{\lambda^{l}}-v w^{\top}\right|<\epsilon \xi<\epsilon_{1} v w^{\top}
$$


since all elements of the rank-1 matrix $v w^{\top}$ are strictly positive. This produces the entry-wise bound,

$$
\left(1-\epsilon_{1}\right) v w^{\top}<\frac{\left(Q \sum V_{i}\right)^{l}}{\lambda^{l}}<\left(1+\epsilon_{1}\right) v w^{\top}
$$

We then have,

$$
\left(1-\epsilon_{2}\right) P_{e s t}<P\left(\left[X_{l+1}=x_{1}, \ldots, X_{l+n}=x_{n}\right]\right)<\left(1+\epsilon_{2}\right) P_{e s t},
$$

where we've used $\|A\|_{\text {en }} \leq\|B\|_{\text {en }}$ whenever $A \leq B$ entry-wise, for non-negative matrices $A$ and $B$.

Let $H_{m, a}, H_{m, b}, H_{m, c}$ denote the entropies of the joint distributions of the left, middle, and right segments respectively.

The entropy associated with the $P_{\text {est }}$ distribution, call it $H_{m, b, e s t}$ may now be related to $H_{m, b}$, the original middle joint entropy. Using the shorthand $P_{a c t}=$ $P\left(\left[X_{l+1}=x_{1}, \ldots, X_{l+n}=x_{n}\right]\right)$,

$$
\begin{aligned}
H_{m, b} & =-\sum_{x_{l+1}, \ldots, x_{l+n}=1, \ldots, k} P_{a c t} \log \left(P_{a c t}\right) \\
& <-\sum\left(1+\epsilon_{2}\right) P_{\text {est }} \log \left(\left(1-\epsilon_{2}\right) P_{\text {est }}\right) \\
& <-\left(1+\epsilon_{2}\right) \sum P_{\text {est }} \log \left(P_{\text {est }}\right)-\left(1+\epsilon_{2}\right) \log \left(1-\epsilon_{2}\right) \\
& <\left(1+\epsilon_{2}\right) H_{m, b, e s t}+\epsilon_{3},
\end{aligned}
$$

independent of $m$, provided $l$ is fixed, and where we've used the fact the probabilities sum to 1 in the second inequality. Similarly for the lower bound.

Since we have a stationary stochastic process, the existence of the entropy rate follows immediately:

$$
\lim _{m \rightarrow \infty} \frac{H_{m, b, e s t}}{m}=h_{r}
$$

Next, by the basic properties of entropy, we have,

$$
H_{m, b} \leq H_{m} \leq H_{m, a}+H_{m, b}+H_{m, c} .
$$

Since $l$ is fixed, the entropies associated with the left and right joint distributions, $H_{m, a}$ and $H_{m, c}$, obey $H_{m, a}, H_{m, c} \leq l \log (k)$ for all $m$ (a maximum of $\log (k)$ per symbol). In total then, by (8.11) and (8.12), the average entropy for the random vectors in $\mathcal{X}$ converges to the same limit:

$$
h_{r} \leq H_{m} \leq \lim _{m \rightarrow \infty} \frac{2 l \log (k)+H_{m, b}}{m}=h_{r},
$$

within an arbitrarily small $1 \pm \epsilon$ factor.

We further have ergodicity:

Theorem 5. The stationary stochastic process of Lemma 6 is ergodic.

Proof. We'll show the process is mixing. For a stationary process it suffices to show that, under the shift map $\sigma$,

$$
\lim _{j \rightarrow \infty} P\left(\left(\sigma^{-n} A\right) \cap B\right)=P(A) P(B),
$$

where $A$ and $B$ are arbitrary basic cylinder sets of form,

$$
C_{-i}\left[x_{-i}, \ldots, x_{j}\right]=\ldots, *, *, x_{j}, x_{j-1}, \ldots, x_{0}, \ldots, x_{-i+1}, x_{-i}, *, *, \ldots
$$


for $i, j \in \mathbb{N}$.

Let $A=C_{-i_{1}}\left[x_{-i_{1}}, \ldots, x_{j_{1}}\right]$ and $B=C_{-i_{2}}\left[y_{-i_{2}}, \ldots, y_{j_{2}}\right]$. Fix $l$. Let $N=i_{1}+j_{2}+2 l$. Then for all $n \geq N$,

$$
\left(\sigma^{-n} A\right) \cap B=\ldots, *, x_{j_{1}}, \ldots, x_{-i_{1}}, \overbrace{, \ldots, *, *, \ldots, *}^{\mathrm{r}}, y_{j_{2}}, \ldots, y_{-i_{2}}, *, \ldots,
$$

where $r \geq 2 l$.

From the proof of Lemma 7 , provided $l$ is chosen large enough, we have the matrix form approximation for the probability of (8.13) of,

$$
\begin{aligned}
& P\left(\left(\sigma^{-n} A\right) \cap B\right) \approx \\
& \quad c_{N}\left\|v w^{\top} Q V_{x_{j_{1}}} \cdots Q V_{x_{-i_{1}}} v w^{\top} Q V_{y_{j_{2}}} \cdots Q V_{y_{-i_{2}}} v w^{\top} Q\right\|_{e n} .
\end{aligned}
$$

The normalization constant is correspondingly,

$$
c_{N}^{-1} \approx\left\|v w^{\top}\left(Q \sum_{i} V_{i}\right)^{i_{1}+j_{1}+1} v w^{\top}\left(Q \sum_{i} V_{i}\right)^{i_{2}+j_{2}+1} v w^{\top} Q\right\|_{e n} .
$$

(All $\lambda$ factors have been factored out.) While for $P(A)$ and $P(B)$ we have the exact form,

and normalization constant,

$$
\left\|v w^{\top} Q V_{z_{j}} \cdots Q V_{z_{-i}} v w^{\top} Q\right\|_{e n}
$$

$$
c_{N}^{-1}=\left\|v w^{\top}\left(Q \sum_{i} V_{i}\right)^{i+j+1} v w^{\top} Q\right\|_{e n},
$$

where $z=x$ and $y$, and $i, j=i_{1}, j_{1}$ and $i_{2}, j_{2}$, for cylinder sets $A$ and $B$ respectively.

We notice that for any matrix $M$ of appropriate dimension,

$$
v w^{\top} M v w^{\top}=v\left(w^{\top} M v\right) w^{\top}=v c_{M} w^{\top}=c_{M} v w^{\top},
$$

where $c_{M}$ is a scalar. This gives,

$$
P\left(\left(\sigma^{-n} A\right) \cap B\right) \approx \frac{\left\|v c_{\alpha} c_{\beta} w^{\top} Q\right\|_{e n}}{\left\|v c_{1} c_{2} w^{\top} Q\right\|_{e n}}=\frac{c_{\alpha} c_{\beta}}{c_{1} c_{2}}
$$

where $c_{\kappa}=w^{\boldsymbol{\top}}\left(Q \sum_{i} V_{i}\right)^{i_{\kappa}+j_{\kappa}+1} v, \kappa \in\{1,2\}$.

Similarly,

$$
P(A) P(B)=\frac{\left\|v c_{\alpha} w^{\top} Q\right\|_{e n}}{\left\|v c_{1} w^{\top} Q\right\|_{e n}} \frac{\left\|v c_{\beta} w^{\top} Q\right\|_{e n}}{\left\|v c_{2} w^{\top} Q\right\|_{e n}}=\frac{c_{\alpha} c_{\beta}}{c_{1} c_{2}} .
$$

Since the error in the approximation of (8.14) goes to 0 as $n \rightarrow \infty$, this proves mixing. Since mixing implies ergodicity, this completes the proof.

By the Shannon-McMillan-Breiman theorem, given a discrete-time stationary ergodic stochastic process over a finite sample space, the asymptotic equipartition property holds. That is, we've shown,

$$
\lim _{n \rightarrow \infty}-\frac{1}{n} \log \left(P\left(X_{1}=x_{1}, \ldots, X_{n}=x_{n}\right)\right)=h_{r},
$$

almost surely, where $h_{r}$ is the entropy rate of the associated stationary stochastic process defined in Lemma 6 . The AEP ensures the total set of sequences in the limit of length $n$ may be portioned into, relatively few, 'typical' sequences, which contain 
the asymptotic entirety of the probability mass, and the rest, which asymptotically have measure zero under the probability measure. This ensures arbitrary $n$-long sequences from the source are compressible to $n h_{r}$ units (bits or nats, etc.), provided $n$ is sufficiently large.

The final step in the single block row case is to extend the equipartition property to the random vectors $\mathcal{X}_{m}$.

Theorem 6. For random vectors $\mathcal{X}_{m}$ under parameter set $\left\{\left\{p_{1}, \ldots, p_{k}\right\}, \delta, \rho\right\}, \delta \in$ $(0,1)$, where the associated matrices, $\left\{V_{i}\right\}_{i}$, consist of only a single block row, we have,

$$
\lim _{m \rightarrow \infty}-\frac{1}{m} \log \left(P\left(X_{1}=x_{1}, \ldots, X_{m}=x_{m}\right)\right)=h_{r},
$$

almost surely, where $h_{r}$ is the entropy rate assured by Lemma 7.

Proof. As with the proof of Lemma 7, consider $n, l>0, m=n+2 l$, and the $m$-long cylinder set,

$$
s_{1}=*, \ldots, *, x_{l+1}, \ldots, x_{l+n}, *, \ldots, *,
$$

where $*$ indicates an open symbol. Also consider the corresponding $m$-long sequence with middle $n$ values identical to those of $s_{1}$,

$$
s_{2}=x_{1}, \ldots, x_{l+1}, \ldots, x_{l+n}, \ldots, x_{m} .
$$

Let $P_{v}$ denote the probability distribution over vectors $\mathcal{X}_{m}$, and $P_{s}$ denote the stationary distribution defined in Lemma 6 . For $l$ sufficiently large,

$$
P_{v}\left(s_{1}\right) \approx P_{s}\left(x_{l+1}, \ldots, x_{l+n}\right),
$$

where the approximation may be made to within a factor of $1 \pm \epsilon$ for $l$ sufficiently large (see (8.10)). Note we've shown the latter distribution, $P_{s}$, to possess the AEP.

Let $A$ be the matrix product corresponding to the middle sequence $x_{l+1}, \ldots, x_{l+n}$ :

$$
A=Q V_{x_{l+n}} \cdots Q V_{x_{l+1}} .
$$

Then

$$
\begin{aligned}
& P\left(s_{1}\right)=c_{N}\left\|\left(Q \sum V_{i}\right)^{l} A\left(Q \sum V_{i}\right)^{l} Q\right\| \approx c_{N}\left\|v w^{\top} A v w^{\top} Q\right\|=c_{N}\left\|B_{l} A B_{r}\right\| \\
& P\left(s_{2}\right)=c_{N}\left\|Q V_{x_{m}} \cdots Q V_{x_{m-l+1}} A Q V_{x_{l}} \cdots Q V_{x_{1}} Q\right\|=c_{N}\left\|C_{l} A C_{r}\right\| .
\end{aligned}
$$

We next note the Perron-derived rank-1 matrix, $v w^{\top}$, is strictly positive, as is $Q$ in either padding case. So $B_{l}$ and $B_{r}$ are strictly positive.

In the pad-and-splice case, $C_{l}$ and $C_{r}$ are strictly positive. In the pad-only case, since $Q$ is strictly positive, $A=Q V_{x_{l+n}} \cdots Q V_{x_{l+1}}$ may be entry-wise bounded between factors of $V_{x_{l+n}} \cdots Q V_{x_{l+1}}$. We also note $C_{l} Q$ and $C_{r}$ are strictly positive. For the splice-only case, $C_{l}$ and $C_{r}$ have a strictly positive diagonal (since $\delta \in(0,1)$ ). This ensures,

$$
\begin{aligned}
d_{1, \min }\|A\| \leq c_{N}\left\|B_{l} A B_{r}\right\| \leq d_{1, \max }\|A\| \\
d_{2, \min }\|A\| \leq c_{N}\left\|C_{l} A C_{r}\right\| \leq d_{2, \max }\|A\| .
\end{aligned}
$$

This produces the bound,

$$
0<c_{\min } P\left(s_{1}\right)<P\left(s_{2}\right)<c_{\max } P\left(s_{1}\right)<1,
$$

for $l$ sufficiently large, where $0<c_{\min }<d_{2, \min } / d_{1, \max }$ and $c_{\max }=$ $\min \left\{d_{2, \max } / d_{1, \min }+\epsilon, 1 / P\left(s_{1}\right)-\epsilon\right\}$. Taking the averages of the logarithm gives,

$$
-\frac{1}{m} \log \left(P_{v}\left(s_{1}\right)\right)-\frac{\log \left(c_{\max }\right)}{m}<-\frac{1}{m} \log \left(P_{v}\left(s_{2}\right)\right)<-\frac{1}{m} \log \left(P_{v}\left(s_{1}\right)\right)-\frac{\log \left(c_{\min }\right)}{m} .
$$


For $s_{1}$, we have by the asymptotic equipartition property for the stationary ergodic form,

$$
\left|-\frac{1}{m} \log \left(P_{s}\left(X_{1}=x_{l+1}, \ldots, X_{n}=x_{l+n}\right)\right)-h_{r}\right|<\epsilon,
$$

almost surely, under $P_{s}$, for all $m$ sufficiently large. By (8.16), where that approximation is within a $1 \pm \epsilon$ factor for $l$ large enough,

$$
\lim _{m \rightarrow \infty}-\frac{1}{m} \log \left(P_{v}\left(s_{2}\right)\right)=h_{r}
$$

a.s. in $P_{s}$. We need this, however, a.s. in $P_{v}$. This follows since the whole space of $m$-long sequences can be decomposed into the disjoint union,

$$
\left(\bigcup_{t \in \text { typical }} * \ldots *[t] * \ldots *\right) \bigcup\left(\bigcup_{t \in \text { atypical }} * \ldots *[t] * \ldots *\right),
$$

where $P_{v}\left(\bigcup_{t \in \text { typical }} * \ldots *[t] * \ldots *\right) \rightarrow 1$ as $m \rightarrow \infty$, by the $P_{v}$ approximation (8.16) to the stationary distribution, $P_{s}$, and the AEP. The a.s. under $P_{v}$ then corresponds to all those vectors, $\mathcal{X}_{m}$, with 'middle' values, $x_{l+1}, \ldots, x_{l+n}$ in the 'typical' set under the stationary distribution, while the 'end' values, $x_{1}, \ldots, x_{l}$ and $x_{l+n+1}, \ldots, x_{m}$, can be arbitrary.

8.2.2. Entropy rate; multiple block rows. We now consider the case where the matrices $\left\{Q V_{1}, \ldots, Q V_{k}\right\}$ have more than one block row. In this case, we'll see that at fixed $m$ the total random vector $\mathcal{X}_{m}=\left(X_{1}, \ldots, X_{m}\right)$ can be approximated by a distributional mixture of individual block row random vectors, each block row's random vector having been shown to haven an entropy rate and possess an AEP, by Lemma 7 and Theorem 6 .

Using the notation of Section 7.8, let $R_{i}=Q V_{i}$, and let $D_{i, j}, i \in\left[1, i_{\max }\right]$, refer to the matrix of block row $i$ of symbol matrix $R_{j}$. Let $\lambda_{i}$ be the eigenvalue of matrix $\sum_{j} D_{i, j}$ (i.e. of the $Q \sum_{j} V_{j}$ matrix resulting from summing over all symbols of block row $i$ ). Let $\lambda_{\max }=\max \left\{\lambda_{1}, \ldots, \lambda_{i_{\max }}\right\}$. Let $V_{\max }$ be the set of block row indices corresponding to block rows with maximal eigenvalue $\lambda_{\max }$ : $V_{\max }=\left\{i: \lambda_{i}=\lambda_{\max }\right\}$.

Fix $m$. We'll consider the total probability distribution defined by normalized products of the $R_{i}$ as a mixture of normalized probability distributions defined by each block row of the $R_{i}$. For $m$-long sequence $s$, the probability is,

$$
P(s)=c_{N}\left\|\left[\begin{array}{ccc}
D_{1, j_{m}} & 0 & \cdots \\
0 & D_{2, j_{m}} & 0 \\
\vdots & 0 & \ddots
\end{array}\right] \cdots\left[\begin{array}{ccc}
D_{1, j_{1}} Q_{1} & 0 & \cdots \\
0 & D_{2, j_{1}} Q_{2} & 0 \\
\vdots & 0 & \ddots
\end{array}\right]\right\|,
$$

where,

$$
c_{N}^{-1}=\left\|\left[\begin{array}{cccc}
\left(\sum_{j} D_{1, j}\right)^{m} Q_{1} & 0 & 0 & \ldots \\
0 & \left(\sum_{j} D_{2, j}\right)^{m} Q_{2} & 0 & \ldots \\
\vdots & 0 & \cdots & \left(\sum_{j} D_{i_{\max }, j}\right)^{m} Q_{i_{\max }}
\end{array}\right]\right\| \text {, }
$$

with $Q_{i}$ denoting the padding matrix belonging to the $i$ th block row. 
Let $c_{N, i}$ denote the normalization constant associated with the $i$ th block row. Then,

$$
c_{N, i}^{-1}=\left\|\left(\sum_{j} D_{i, j}\right)^{m} Q_{i}\right\| \rightarrow \lambda_{i}^{m}\left\|v_{i} w_{i}^{\top} Q_{i}\right\|=a_{i} \lambda_{i}^{m},
$$

as $m \rightarrow \infty$, within error factor $1 \pm \epsilon$, by the proof of Lemma 7 .

We now write $P(s)$ as a mixture of the individual block row probability distributions:

$$
P(s)=\frac{c_{N, 1}^{-1}\left(c_{N, 1}\left\|D_{1, j_{m}} \cdots D_{1, j_{1}} Q_{1}\right\|\right)+c_{N, 2}^{-1}\left(c_{N, 2}\left\|D_{2, j_{m}} \cdots D_{2, j_{1}} Q_{2}\right\|\right)+\ldots}{c_{N, 1}^{-1}+c_{N, 2}^{-2}+\ldots} .
$$

The mixture weighting is a function of $m$. Observe that as $m \rightarrow \infty$, by (8.17), the block row probability distributions corresponding to block rows with maximum eigenvalue, i.e. those indexed by $V_{\max }=\left\{n_{1}, \ldots, n_{f}\right\}$, will asymptotically dominate by a scaling factor: $\left(\lambda_{\max } / \lambda_{l}\right)^{m}$ grows without bound whenever $l \notin V_{\max }$. So when considering approximations to the probability distribution in the multiple block row case, for sufficiently large $m$ it suffices to restrict attention to the block rows with maximal $Q \sum V_{i}$ eigenvalue:

$$
P(s) \approx c_{1} c_{N, n_{1}}\left\|D_{n_{1}, j_{m}} \cdots D_{n_{1}, j_{1}} Q_{n_{1}}\right\|+\ldots+c_{f} c_{N, n_{f}}\left\|D_{n_{f}, j_{m}} \cdots D_{n_{f}, j_{1}} Q_{n_{f}}\right\|
$$

where $c_{i}=c_{N, n_{i}}^{-1} / \sum_{l} c_{N, n_{l}}^{-1}$.

At this point we could modify Lemma 6 , with $B=\sum_{i} A_{i}$ representing a block row matrix with each block row having the same Perron-Frobenius eigenvalue. The approach of Lemma 7 could be copied, applying it exclusively to the block rows indexed in $V_{\max }$. This would prove stationarity of the middle cylinder of Figure 16. Similar $\epsilon$ arguments, bounding the error over all block rows, would lead to the existence of the entropy rate. Alternatively, we can simply use a common information theoretic argument for the entropy rate of a mixture. This approach is used to prove the following fundamental theorem on the entropy rate in the most general case.

Theorem 7. For random vectors $\mathcal{X}_{m}$, under the distribution associated with regularity parameter set, $\left\{\left\{p_{1}, \ldots, p_{k}\right\}, \delta, \rho\right\}, \delta \in(0,1)$, the limit,

$$
\lim _{m \rightarrow \infty} \frac{H\left(\mathcal{X}_{m}\right)}{m},
$$

exists.

If, further, the $Q V_{i}$ matrices have more than one block row, let $V_{\max }=$ $\left\{n_{1}, \ldots, n_{f}\right\}$ denote the set of block row indices corresponding to the maximum $\sum_{i} Q V_{i}$ block row eigenvalue. Let $h_{r, n_{i}}$ denote the entropy rate of block row $n_{i}$. Then the total entropy rate is the weighted average of the entropy rates corresponding to the maximal eigenvalue block rows:

$$
h_{r}=c_{1} h_{r, n_{1}}+\ldots+c_{f} h_{r, n_{f}},
$$

where $c_{i}=\lim _{m \rightarrow \infty} c_{N, n_{i}}^{-1} / \sum_{l} c_{N, n_{l}}^{-1}$.

Proof. The proof for the case when the $Q V_{i}$ are composed of a single block has been covered by Lemma 7 . 
For the multiple block row case, let $Y_{n_{i}, m}$ represent the $m$-long random vector corresponding to block row $n_{i}$. By (8.18), we are then interested in showing the entropy rate of the dominant mixture,

$$
P(Z)=c_{1} P\left(Y_{i_{1}, m}\right)+\ldots+c_{j} P\left(Y_{i_{j}, m}\right),
$$

exists, where weight $c_{i}=\lim _{m \rightarrow \infty} c_{N, n_{i}}^{-1} / \sum_{i} c_{N, n_{i}}^{-1}$ (by (8.17) the $c_{i}$ ratios stabilize asymptotically in $m$ ).

We then note,

$$
\sum_{i} c_{i} H\left(Y_{n_{i}, m}\right) \leq H\left(\sum_{i} c_{i} Y_{n_{i}, m}\right)=H(Z)
$$

by concavity of the entropy. Treating index variable $I$ as a generalized Bernoulli random variable with probability $P(I=i)=c_{i}$, we also have,

$$
H\left(\sum_{i} c_{i} Y_{n_{i}, m}\right)=H(Z) \leq H(Z \mid I)+H(I)=\sum_{i} c_{i} H\left(Y_{n_{i}, m}\right)-\sum_{i} c_{i} \log \left(c_{i}\right) .
$$

Dividing by $m$, and using the existence of the single block row entropy rates by Lemma 7 , a pinching argument shows the entropy rate exists and is equal to,

$$
h_{r}(Z)=\sum_{i} c_{i} h_{r, n_{i}} .
$$

In the single block row case, by Theorem 6 , we saw the distributions possessed an AEP. In the multiple block row case, in the event there is more than one maximal eigenvalue block row, the mixture of (8.18) produces a union of rare 'typical' sequences that hold the majority of the probability mass (we offer no assurance the union is disjoint).

A final remark is in order on the potential equivalence between the block rows corresponding to the maximal $Q \sum V_{i}$ eigenvalue, indexed by $V_{\max }$, and the 'maximal' block rows of Section 7.8, corresponding to the maximal Lyapunov exponent, indexed by $U_{t^{\prime}}$. Since all matrices are non-negative, a relatively large block row $Q \sum V_{i}$ matrix eigenvalue should correspond to a large $\sum_{i} S_{i}$ norm-i.e. a block row with a relatively large number of fully regular template sequences encoded in the $S_{i}$. This should also correspond to a relatively large block row Lyapunov exponent, since the norm of the matrix product corresponding to a given sequence is larger the more ways there are to create fully regular sequences out of sub- and supersequences of the given sequence. Though more detailed reasoning is not offered, this suggests the 'maximal' block row index sets $U_{t^{\prime}}$ and $V_{\max }$ in many or all cases may be the same.

In any case, if $V_{\max }$ is equal to an equivalence class, $U_{t}$, of Section 7.8, then, by definition of the equivalence classes, the entropy rates of all rows indexed by $V_{\max }$ are equal, and the total entropy rate is simply equal to the entropy rate of any of the maximal block rows, by Theorem 7 .

8.3. Monte Carlo techniques for entropy. In aim of applying Monte Carlo techniques to estimate entropy rates, consider the discussion near the end of Section 4.1. As $\delta \rightarrow 0$, samples that contribute significantly to $\left\|S_{m}\right\|_{e n}$, and by extension $\left(\left\|S_{m}\right\|_{e n} / n\right) \log \left(\left\|S_{m}\right\|_{e n} / n\right)$, become increasingly rare. The AEP result of Theorem 6 , emphasizes this situation. The proportion of 'typical' sequences to total 
sequences may approach zero asymptotically in $m$, while the typical sequences also retain the majority of the probability mass.

Since obtaining a sample entropy requires sampling sequences with the bulk of the probability mass, this means ordinary brute force Monte Carlo (BFMC) can become prohibitively time consuming: finding samples that meaningfully contribute to the quantity under estimation becomes exceedingly difficult at $m$ sufficiently large.

In [58], Vanneste creates a form of importance sampling to overcome a similar difficulty in numerically estimating generalized Lyapunov exponents of random matrix products, these being the expectation values of matrix product norms raised to the $q$ th power. With a slight modification, this technique can be applied equally well to the numerical estimates for entropy here. The algorithm follows that in [58] very closely.

We initialize with a sample size of $L$ matrices at the first iteration step, assigning the padding matrix, $E_{1}^{(l)}=P$ (I in the case of splice-only), $l \in[1, L]$, and initialize entropy values at the zeroth iteration step as $H_{0}^{(l)}=1$. At each successive iteration, $t, L$ matrices are chosen with equal probability from $\left\{P V_{1}, \ldots, P V_{k}\right\}$, and the matrix products are computed, $F_{t}^{(l)}=M_{i} E_{t}^{(l)}$, along with the associated entropy values, $H_{t}^{(l)}=-\left(c_{N}\left\|F_{t}^{(l)}\right\|_{e n}\right) \log \left(c_{N}\left\|F_{t}^{(l)}\right\|_{e n}\right)$. We then resample according to entropy growth:

$$
\alpha_{t}^{(l)}=\frac{H_{t}^{(l)}}{H_{t-1}^{(l)}}=\frac{\left(c_{N}\left\|Y_{t} \cdots Y_{1}\right\| \log \left(c_{N}\left\|Y_{t} \cdots Y_{1}\right\|\right)\right.}{\left(c_{N}\left\|Y_{t-1} \cdots Y_{1}\right\| \log \left(c_{N}\left\|Y_{t-1} \cdots Y_{1}\right\|\right)\right.}
$$

Computing $\beta=\sum_{l} \alpha_{t}^{(l)}$, we define random variable $J$ by $P(J=j)=\alpha_{t}^{(j)} / \beta$. We then resample,

$$
E_{t+1}^{(l)}=F_{t}^{(J)}
$$

This naturally favors matrix products with larger increases in entropy over the previous step. Since the $\left\{P V_{1}, \ldots, P V_{k}\right\}$ matrix selection is equiprobable, each product has probability $1 / k^{m}$. This allows expressing the estimate of the expectation value of the entropy, through estimator $Z_{m}=\beta_{1} \cdots \beta_{m} / L^{m}$ (see [58]), in terms of the total entropy of the distribution associated with the (normalized) matrix product norms (with normalization constant $c_{N}$ given by (7.14)):

$$
\mathbb{E}\left(\left(c_{N}\left\|S_{m}\right\|_{e n}\right) \log \left(c_{N}\left\|S_{m}\right\|_{e n}\right)\right)=\sum \frac{\left(c_{N}\left\|S_{m}\right\|_{e n}\right) \log \left(c_{N}\left\|S_{m}\right\|_{e n}\right)}{k^{m}} .
$$

The estimator for the total entropy, (8.1), is then simply,

$$
H(p, \delta) \approx \frac{k^{m}}{L^{m}} \beta_{1} \cdots \beta_{m} .
$$

In [58], Vanneste gives rigorous proof that the estimator for the expectation value of the norms' $q$ th power is unbiased. This is also the case here. The proof in [58] need only be modified by the exchange of Vanneste's $\alpha$ 's, which are the ratio of the $q$ th powers of the norms between the present step and the past step, for the entropy ratios in (8.19). Vanneste also offers justification for why this go-with-the-winners strategy produces a much lower sample variance. The result of modifying the associated computation of $\mathbb{E}_{p r} \mathbb{E}_{p t}\left(Z_{m}^{2}\right)$ in [58] with these new $\alpha$ 's, where $p r$ denotes the expectation over all matrix products, and $p t$ denotes the expectation over all possible (resampling) paths, merely introduces additional 
factors of magnitude $\log \left\|A_{n} \cdots A_{1}\right\|$ in the overall $Z_{m}^{2}$ expectation value. These factors have little effect on the overall sharp reduction in variance the technique provides.

In Figure 17 are entropy curves plotted vs. $\delta$ for three cases for a binary alphabet. All are from the splice-only distribution. The lowest shows the results of the resampling method at $5 e 4$ samples, plotted against the actual entropy curve at $m=20, p=3 / 5$. The two curves are indistinguishable. The middle and upper curves are for $p=4 / 5$ and $p=3 / 5$ respectively, both at $m=100$ and $1.5 e 5$ samples. The boxed endpoints in these two cases mark the exact computed entropy at the $\delta \in\{0,1\}$ extremes, as above.

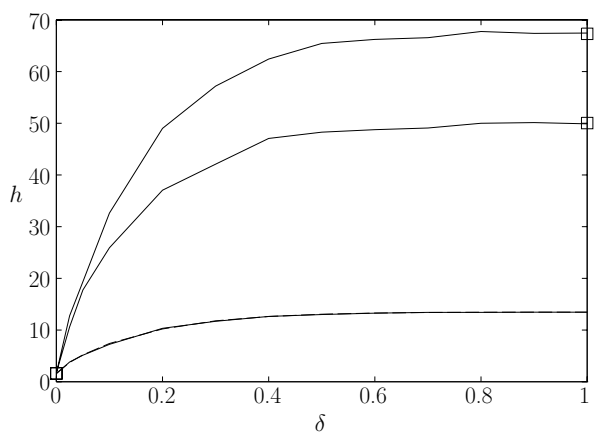

FiguRE 17. entropy curves for splice-only, from the resampled Monte Carlo routine; lowest curve is $m=20, p=3 / 5,5 e 4$ samples, with actual curve superimposed (indistinguishable); middle and upper curves are $p=4 / 5$ and $p=3 / 5$ respectively, at $m=100$ and $1.5 e 5$ samples each

For parameter set, $\left\{\left\{p_{1}, \ldots, p_{k}\right\}, \delta, \rho\right\}$, with symbol probabilities, $p_{1}, \ldots, p_{k}$, fixed, we can consider the random vector entropy rate, $h_{r}$, as a function of $\delta$ and $\rho$. Figure 18 shows the results of applying resampled Monte Carlo to estimate entropy rates over such a region. Parameters were $p=3 / 5$, pad-and-splice, where $\delta \in[0,1]$, and $s$ ranges between the minimal value, 5 , up to 20 .

The $\delta$ parameter can be interpreted as moderating between pure topological entropy at the $\delta=0$ extreme, and pure Bernoulli entropy, $h_{r}=-(3 / 5) \log (3 / 5)-$ $(2 / 5) \log (2 / 5)$, at the $\delta=1$ extreme (for all $s$ ). The entropy rate actually rises slightly from the Bernoulli entropy in the region $\delta \approx[0.5,0.95]$, before falling off as $\delta \rightarrow 0$.

The values of $h_{r}$ in the region may also be considered as the degree of (heterogeneous) 'surprise' away from the purely homogeneous $\overline{10110}$ templates (and their circular permutations), i.e. away from $h_{r}$ in the lower left corner of the plot $(\delta=0, s=5)$, which measures the entropy rate of the templates themselves (with $s$ minimal, this being $h_{r}=0$ ).

\section{Properties of the measures}

Some basic properties of the z-score measures, and their suitability as an overall sequence regularity statistic will be examined, with a focus on binary sequences. The general results will hold for longer alphabets as well. 


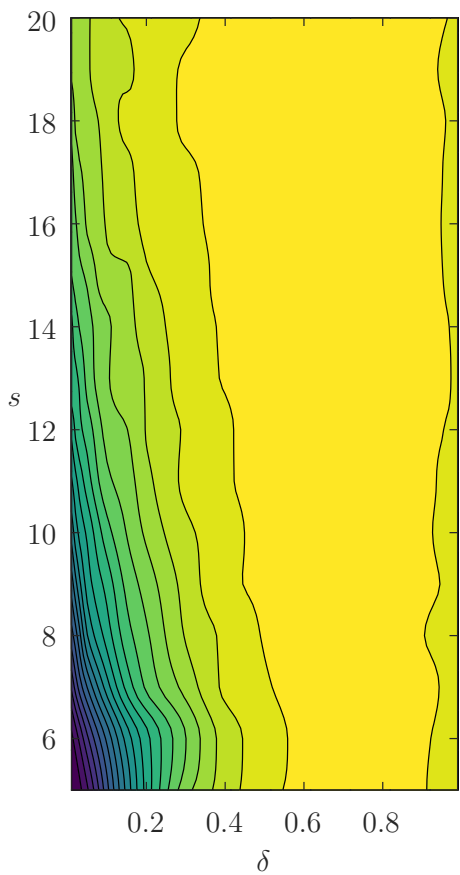

Figure 18. (color online) approximate level curves for the entropy rate, $h_{r}$, for a binary alphabet at $p=3 / 5$, over the region $\delta \in[0,1]$, $\rho=s \in[5,20] ;$ note the $\delta$ axis is continuous, while the $s$ axis is discrete; cubic interpolation was employed to produce smooth topography; along the right margin, $h_{r} \rightarrow-(3 / 5) \log (3 / 5)-$ $(2 / 5) \log (2 / 5)$ as $\delta \rightarrow 1$ for all $s$; some waviness in the contours is likely an artifact of processor limitations

First, note that up to now the binary sequences have been relatively short, producing z-scores more or less within familiar ranges. As sequence length expands, however, z-scores of fully regular or near-fully regular sequences can become quite large. This is an artifact of the central limit theorem's mean decreasing without bound as $\delta \rightarrow 0$, while sequences with high regularity can stay near 0 (see the discussion around the lognormal distribution, near the end of Section 4.1). This will be evident in some of this section's plots.

9.1. Forms of variance. We'll first examine how z-score measures differ from forms of statistical variance. Two types of variance will be considered.

Given binary sequence $S$, let $\left\{n_{l}\right\}$ index the positions of the 1 bits. Then $I_{S}=$ $\left\{n_{l+1}-n_{l}\right\}$ is simply the set of inter-1 distances of $S$. Let the inter-1 variance be the statistical variance of set $I_{S}$ :

$$
\operatorname{Var}_{1}=\operatorname{Var}\left(I_{S}\right)
$$

Let $C(j)=\sum_{j=1}^{m} s_{j}$ be the discrete function representing the cumulative sum of bits in $S$. Then we define the $q$-slope variance as the variance of the set of differences between values in $C(j)$ and a line of slope $q$ :

$$
\operatorname{Var}_{q}=\operatorname{Var}\left(\{C(j)-q j\}_{j}\right) .
$$

The $q$-slope variance is designed to be smallest when the sequence is fully $(p)$ regular. It is directly related to (binary) sequence discrepancy, taking the measure of sequence discrepancy as the sum rather than the max: $\sum_{i=1,2 ; t \in[1, m]}\left|Z_{i}(t)-p_{i} t\right|$. 
9.2. Effects of noise. The noise plot, Figure 19, shows the effects of salt-andpepper noise on an initially fully regular sequence $(p=4 / 5)$, of length $m=200$. Each of the three lower curves is the z-score under $\delta=0.1$ of each of the three main methods developed: splice-only, pad-only, and pad-and-splice. The noise is generated by over-writing randomly chosen bits, with the overwrite bit chosen by Bernoulli weighting $(\mathrm{P}(0)=p)$. The $\mathrm{x}$-axis denotes the number of overwrites. After around 100 overwrites, most of the regularity has been driven out of the sequence under all three scoring methods (z-scores near 0 ). The two curves above the three lower ones are the $q$-slope variance (magenta) and the inter- 1 variance (light blue), both rescaled for comparison purposes; the large fluctuations in both variance curves vs. the more stable behavior of the z-scores suggests the z-scores are computing something more than variance of these types. Correlation coefficients between the pad-and-splice z-score and the two variance measures, as a function of the number of overwrites (200 total), were averaged over 50 runs each. Between the pad-and-splice $\mathrm{z}$-score and the inter- 1 variance, the correlation averaged $\approx-0.63$. Between the pad-and-splice $\mathrm{z}$-score and $q$-slope variance, the average was $\approx-0.40$. The z-scores should move roughly in the opposite direction of variance.

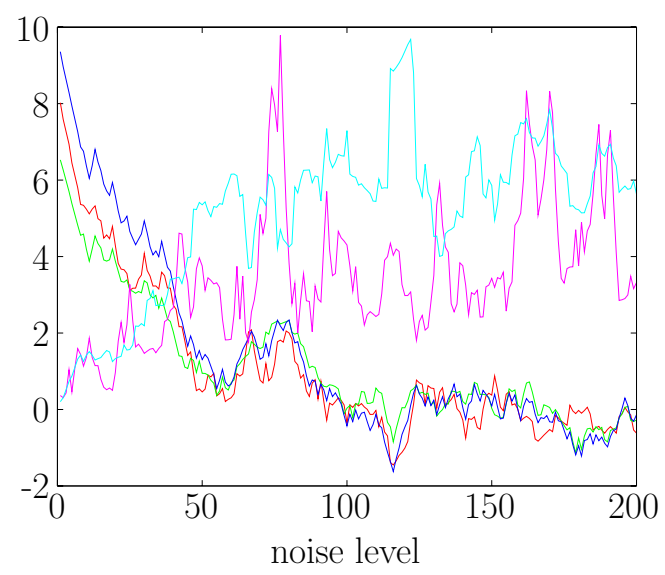

FIGURE 19. (color online) noise plot; a fully regular sequence overwritten with Bernoulli-random $0 s$ and $1 s$; three lower curves at $x>75$ are the $z$-scores; upper two curves are inter-1 variance (light blue) and q-slope variance (magenta)

9.3. Displacing a given bit. Figure 20 shows the effects of displacing a midpoint 1 in an otherwise fully regular sequence with $p \approx 1$. In particular, given a fully regular sequence on $p=19 / 20, m=240$ long (so, 12 concatenated segments of type 10...0, a 1 followed by 19 zeros), the 11th 1 (201st bit) is 'displaced' to the right by $x$ bits (the plot's horizontal axis; e.g. ...0100... under $x=1$ displacement becomes ...0010...). The z-scores from all three methods, along with their average, are computed and plotted (solid lines). The horizontal dashed lines are the respective z-scores and their average, of the sequence with the 201st bit overwritten by a 0 (i.e. the 11 th 1 
bit has been removed entirely, replaced by a 0 ). Note the initial drops in the spliceonly and pad-only plots: displacing the 1 bit by one or more positions requires at least 20 splices or pads to make the sequence fully regular again.

The pad-and-splice curve has a gentler drop because, to first order, we need only one pad and one splice to return the interval to fully regular-splice the 1 from its $x$-offset position, and pad a 1 at the midpoint. Consulting the general $\delta$ polynomial equation (5.9), this amounts to an $l_{s}+l_{p}=2$ term with $\delta$ weight $\delta^{2}(1-\delta)^{239}$. This is true for any $x \in[1,19]$. The gradual drop is due to secondary effects. The next least-costly way to correct the displaced 1 is to splice $k 0$ 's to its left, and pad $k 0$ 's to its right, with $\left(\begin{array}{c}19+k \\ k\end{array}\right)$ and $\left(\begin{array}{c}19 \\ 19-k\end{array}\right)$ ways to do so (for the second value, we have exactly $k$ pads to place among $n-k$ slots, where more than one pad can go in a slot; the padding case can therefore be treated as a weak composition). This produces a $l_{s}+l_{p}=2 k$ polynomial term with $\delta$ weight $\delta^{2 k}(1-\delta)^{240-k}$. This way, the sum of the primary and secondary costs increases as $k$ increases (the cost increases as the sum of the terms in (5.9) decreases, producing a lower norm). The curve levels off as the sheer multiplicity of choices for splicing on the left and padding on the right outweighs the increasing $\delta$ factor penalties as a function of $x$.

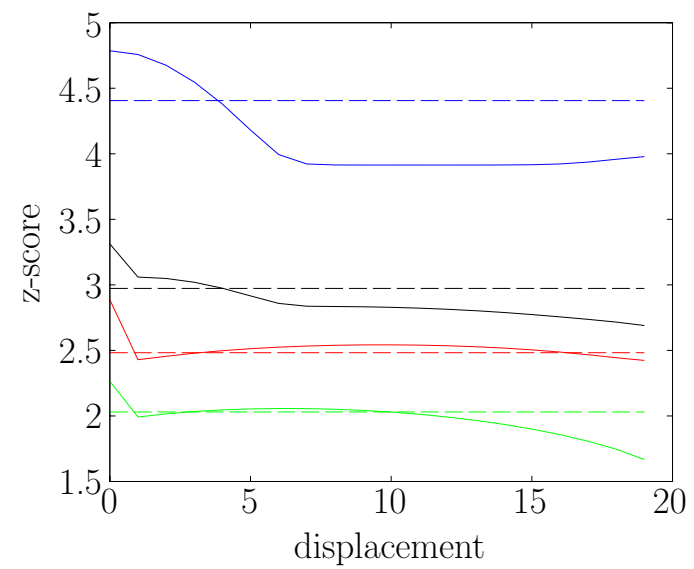

FiguRE 20. (color online) effects of midpoint displacement; padand-splice is at top, pad-only is at bottom, with splice-only just above it; the average $z$-score is 3 rd from bottom; dashed line is $z$-score with the middle bit removed entirely (set to 0)

9.4. Shuffling segments of fixed types. Let $T$ be a set of $r$ sequences of type $10 \ldots 0$, each of length $l_{i}, i \in[1, r]$. Choose $n_{1}, \ldots, n_{r}, n_{i_{1}} \approx n_{i_{2}}$, sequences of each type, and concatenate into a string of total length $\sum_{i} n_{i} l_{i}$. We're interested in the effects on z-scores of shuffling the order of the $10 \ldots 0$ segments in the concatenation.

Figure 21 depicts such a case. We start with a set of binary strings of type $10 \ldots 0$, of lengths $16,18,21,25$ (chosen so their average length is 20 ), and we concatenate approximately equal numbers of each to form a sequence of total length $m=1 e 4$. Initially the component strings are in increasing order of length (so, joining around 120 strings of length 16 , followed by $\approx 120$ strings of length 19 , and so on). A segment shuffle counts as randomly choosing two different $10 \ldots 0$ segments 
in the total sequence and swapping them. All three z-scores (and the average) with $p=19 / 20, \delta=0.1$, are plotted as a function of the number of shuffles. The lowest line in Figure 21 is the $q$-slope variance, rescaled for comparison purposes. (The inter-1 variance is of course a constant of this shuffling process, and so would have a flat line (not shown).) The pad-only z-score is effectively unchanged by the shufflings, while the splice-only scores show a fairly steady monotone decline. The pad-and-splice scores in fact also have variations, but they are on the order of $0.1 \%$ and can't be seen at the present scale. (In this particular example, the padand-splice scores trend upward, implying slightly more regularity from the shuffling process.) The average z-score reflects these characteristics, with a monotone decline, though more muted than splice-only alone. The $q$-slope variance also shows a steady decline, but is more convex; it is also generally smoother than the z-score average. Note that a decreasing $q$-slope variance would imply the sequence in some sense is growing more regular, while the z-score average's declining under the same shuffling process would imply the sequence is growing less regular against the $p$-regular fully regular sequences.

To show why splice-only z-scores tend to decrease with shuffling, assume $p$ of form $(n-1) / n$ and two different basic segments of type 10...0: segment $a$ of total length $n-\alpha$, and segment $b$ of total length $n+\beta, \alpha, \beta>0$, so that under some fixed total number of $a$ 's and $b$ 's we may construct a sequence of length $m$, with an approximately Bernoulli proportion $p$ of zeros to $m$. Consider a subsequence of type $a \ldots a b \ldots b$, with $j_{a} a$ 's followed by $j_{b} b$ 's. We want to determine the first order coverings (see Section 2.3), that is those fully regular subsequences that are the longest possible within the main sequence. The number of splices required to obtain such subsequences will give a first order estimate on the corresponding zscore. This value will be determined in pieces as follows: since $a$ can't be spliced at all to fully regular, we need to consider pairs- $a a$ needs $2(n-\alpha)-n=n-2 \alpha$ splices; $b$ simply needs $\beta$ splices; and note that $a b$ needs $2 n-\alpha+\beta-n=n+\beta-\alpha$ splices. Now if $j_{a}$ is even, the lowest number of splices on the whole block is,

$$
\frac{j_{a}}{2}(n-2 \alpha)+j_{b} \beta,
$$

while if $j_{a}$ is odd, the most efficient way to splice to fully regular is to match the rightmost $a$ with its neighboring $b$, to yield $\left(j_{a}-1\right) / 2 a a$ pairs, followed by an $a b$, followed by $j_{b}-1 b$ 's, which gives

$$
\frac{j_{a}-1}{2}(n-2 \alpha)+n+\beta-\alpha+\left(j_{b}-1\right) \beta=\frac{j_{a}}{2}(n-2 \alpha)+j_{b} \beta+\frac{n}{2},
$$

splices total in this case. They differ by the amount $n / 2$. Given an initial $m$ long ordered sequence $a \ldots a b \ldots b$, random shuffling will start to create shorter $a \ldots a b \ldots b$ subsequences. Note that concatenating such subsequences, we may simply add the splice cost of each component subsequence. Next note that by randomness, it's about as likely as not that $j_{a}$ is odd for each such subsequence. For each such $j_{a}=$ odd occurrence, there is the additional requirement of $n / 2$ more splices. So shuffling, on average, will increase the number of splices required to achieve the maximal fully regular subsequence by roughly $(n / 2)\left(c_{s} / 2\right)$ where $c_{s}$ is the number of separate $a \ldots a b \ldots b$ subsequences. The z-score under splice-only will decrease in proportion. Note though it's certainly possible to contrive mixed sequences that don't decrease in z-score (simply by requiring all $a \ldots a b \ldots b$ subsequences have $j_{a}$ even). The argument can be extended to $>2$ component segments. 
For pad-only under shuffling, it is easy to see that the number of pads required to make any ordering of a fixed number of $a$ 's and $b$ 's fully regular is independent of the ordering, up to the last segment; the $a$ s and $b$ s may be treated independently. In the $a, b$ example, if the last segment is $a$, then this is a perfectly acceptable suffix to a fully regular sequence of type $\overline{10 \ldots 0}$. If the last segment is $b$, however, it requires a padding 1 in between its $n$th and $(n+1)$ th bits. As the total sequence length $m$ increases, the relative contribution due to variations in the last segment are driven to 0 .

Though too small to see in Figure 21, the pad-and-splice curve does have fairly complex variations. Pad-and-splice under shuffling is best understood by considering two lower order 'extremes.'

One extreme is derived by splicing all $j_{a}+j_{b} 1$ s from the $a \ldots a b \ldots b$ sequence (preor post-shuffle), then padding with enough $1 \mathrm{~s}$, approximately $j_{a}+j_{b}$, to make the result fully regular. There are only at most $n$ ways to do this, and the $\delta$ factor in the polynomial of $(5.9)$ at $l_{s}+l_{p} \approx 2\left(j_{a}+j_{b}\right)$ is $\mathcal{O}\left(\delta^{2\left(j_{a}+j_{b}\right)}(1-\delta)^{m-\left(j_{a}+j_{b}\right)}\right)$. At the other extreme, we treat each $a$ and $b$ separately, padding each $a$ with $\alpha$ os, and splicing $\beta 0$ s from each $b$, to make each fully regular. There are $j_{a}\left(\begin{array}{c}19+k \\ k\end{array}\right)+j_{b}\left(\begin{array}{c}19 \\ 19-k\end{array}\right)$ ways to do this. The $\delta$ factor at $l_{s}+l_{p}=\alpha j_{a}+\beta j_{b}$ is $\delta^{\alpha j_{a}+\beta j_{b}}(1-\delta)^{m-\beta j_{b}}$. Note that both extremes are immune to shuffling. We then consider second order effects that result from hybrids of the two extremes: in some regions 1s are manipulated to make that section regular, and in other regions 0s are manipulated. If a given shuffled region of $a$ s and $b$ s happens to have length a multiple of $n$, then the section can be made fully regular just by manipulating 1 s (no need to pad or splice 0 s in addition). Since the 0 manipulation on such a stretch would cost the same regardless of the $a$ and $b$ ordering, the net effect is secondary fluctuations depending on shuffling order.

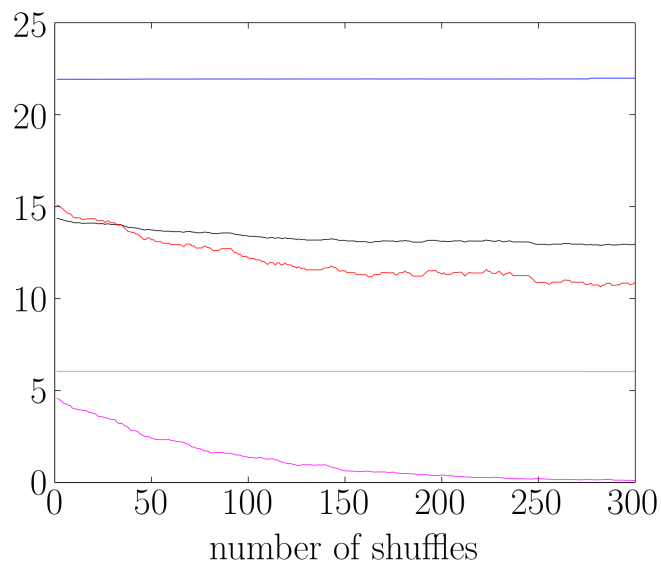

FIGURE 21. (color online) effects of shuffling $k=4$ segments of a fixed type; upper line is pad-and-splice, 2nd and 3 rd from bottom are pad-only and splice-only respectively; 2 nd from top is the z-score average; lowest is q-slope variance; though not visible, up close the pad-and-splice curve has slight variations; the inter-1 variance (not shown) would be a flat line 
9.5. Simple periodic sequences; varying the inter-1 separation. Figure 22 presents a plot of the effects of varying the inter- 1 distance on a sparse binary sequence. Given a binary sub-sequence of type $10 \ldots 0$ of length $x$, create a total sequence of fixed length $m=500$ by concatenation (so e.g. $x=10$ corresponds to $\overline{1000000000}$ ). Using a fixed regular template at $p=19 / 20$ we then compute all three z-scores, and their average, as a function of inter-1 separation distance $x$. This is done at two different $\delta$ values, 0.05 and 0.1 . Each of the four sets of plot lines correspond to one of the three z-scores, and their average. The dashed line in each pair corresponds to $\delta=0.05$, and the solid line to $\delta=0.1$. The initial (leftmost) peak reflects the fully regular sequence, $x=20$. The pronounced subsequent periodicity for both padding cases reflects the low cost of padding a sequence that is regular other than some missing 1-bits, to full regularity (the peaks at $x=40, x=60$, and $x=80$ ). The splice-only score meanwhile steadily declines. The comparatively small difference between curve pairs at $\delta=0.05$ and $\delta=0.1$ suggests the z-scores' relative stability as a function of $\delta$.

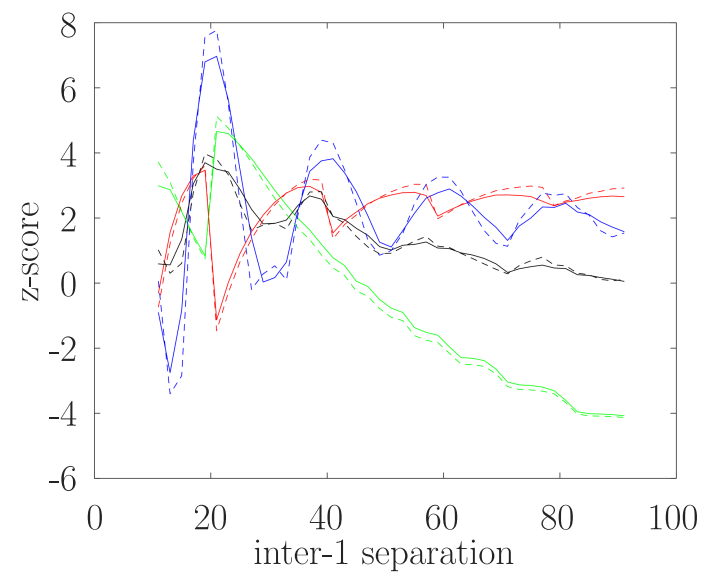

FiguRE 22. (color online) effect of changing inter-1 separation on segments of type $\overline{1 \ldots 0}$ under $p=19 / 20$; solid curves are $\delta=0.1$, dashed curves are $\delta=0.05$; curve with the largest initial peak is pad-and-splice; the curve with the sharp drop at $x=20$ is spliceonly; the pad-only curve has a sharp peak at $x=20$ then steadily declines; the remaining curve is the average

9.6. Periodicity in general. To examine the z-scores of binary periodic sequences in general, let sequence $S$ as usual be of length $m$, and suppose $S=Q Q \ldots Q$, where $Q$ is an arbitrary binary subsequence of length $t, m=j t$. Let $\alpha=\log (\rho)$ denote the log of the spectral radius of $Q$. Then if $T$ is the corresponding matrix product, $j \alpha \leq \log (\| T||)<j(\alpha+\epsilon)$ by the proof of Gelfand's formula. Let $\lambda$ as usual be the Lyapunov exponent associated with the matrix pairs at the given value of $d_{0}, d_{1}$, and $s$, and let $\sigma$ be the variance. Then for the z-score of $S$ we have,

$$
\frac{j \alpha-m \lambda}{\sqrt{m \sigma}} \leq z(S)<\frac{j(\alpha+\epsilon)-m \lambda}{\sqrt{m \sigma}} \Rightarrow\left(\frac{\alpha-t \lambda}{\sqrt{t \sigma}}\right) \sqrt{j} \leq z(S)<\left(\frac{\alpha-t \lambda+\epsilon}{\sqrt{t \sigma}}\right) \sqrt{j} .
$$


In other words, provided $\alpha-t \lambda \neq 0$, the magnitude of the associated z-score will grow without bound as $j$ increases (it may be $<0$ or $>0$, depending on the sign of $\alpha-t \lambda)$.

Now since in general $\log \left\|S_{m}\right\|$ is normally distributed about mean value $m \lambda$, almost all choices of $Q$ will see the $\log$ of the norm of the matrix product corresponding to $Q \cdots Q$ lie outside any sufficiently small neighborhood of $m \lambda$. This shows that for sufficiently large $Q$, which drives $\epsilon \rightarrow 0$, the probability that $j \alpha-m \lambda=0$, and therefore that $\alpha-t \lambda=0$, goes to zero. In the limit of the length of $Q$, and value $m$, periodic sequences will have z-scores whose magnitudes grow without bound, with probability 1.

9.7. Measures and definitions of regularity. The regularity parameter set, $\left\{\left\{p_{1}, \ldots, p_{k}\right\}, \delta, \rho\right\}$, as well as the method employed, splice-only, pad-only, or padand-splice, each relate to sequence regularity.

Each of the individual padding or splicing approaches by themselves may have their own applications. Figures 20, 21, and 22 and the associated discussions help illustrate some of their individual features.

The inter-1 separation plot, Figure 22, especially highlights their differences. There is a marked change in the z-scores when shifting from a sequence that is a predominately padded template sequence, to one that is primarily a spliced template sequence. A semi-homogeneous source that tends only to drop symbols might be well-suited for modeling with a splice-only distribution. Similarly, a source that tends only to intersperse spurious symbols within a semi-homogeneous sequence might be modeled with a pad-only distribution.

In the general case, if we want a measure of symbolic sequence regularity or homogeneity, then there are two main considerations,

What constitutes a fully regular sequence:

- the proportions of each symbol, parametrized through the $p_{i}$

- the proportion spread, parametrized through $s$ relative to $d_{0}+d_{1}\left(d_{0} \leq d_{1}\right)$ in the binary case, and through $h$ in the $(k-1)$-simplex case, $k \geq 2$.

How to measure a given sequence's closeness to a fully regular sequence:

- the processes of splicing or padding as fundamental operations

- the parameter $\delta$ to weight the cost of the fundamental operations in general, and the $p_{i}$ generalized Bernoulli weighting to inform symbol proportions

Ideally, a general measure of sequence regularity should not favor a supercover over a subcover, or vice-versa. Taking the binary example of $p=4 / 5, s=5$, where cyclic rotations of $\overline{10000}$ are fully regular, we'd like runs of type $\overline{1000}$ to be weighted about the same as runs of type $\overline{1000000}$, since they are (roughly speaking) of about equal 'closeness' to the fully regular sequence.

It is natural, then, to assume some combination of padding and splicing will be required. The two main candidates are pad-and-splice by itself, and the average of all three methods.

Figures 20, 21, and 22 help show some of the differences between the pad-andsplice z-score, and the averaged z-score, over all three methods. In all three figures, the average z-score has a stronger downward trend, under the respective effects of midpoint displacement, shuffling, and changing the inter-1 separation in a $p \approx 1$ binary sequence. In the first two, it is because pure splicing is noticeably more costly 
than either padding or pad-and-splice. In the third, it is because pure padding is more costly than the other two methods.

Overall, the benefits of the average z-score over pad-and-splice alone is somewhat open to debate. As in the discussion of Section 9.4, where segment shuffling is considered, we might consider that splice-only, by itself, is in a sense inefficient with respect to total regularity, being unable to see the possibility of padding to make the sequence regular. As a result we might safely discount the trends observed in the average score in the shuffling plot, as well as the midpoint displacement plot. Similarly, in the inter-1 separation plot the inefficiency of padding as the separation increases might be discounted. In some sense, the average risks combining two less-efficient processes with a more efficient one (pad-and-splice, by itself). Otherwise, there is little to suggest whether one is necessarily superior to the other. The applications below have employed pad-and-splice, if at least for computational savings over calculating all three z-scores. Of course all three may nonetheless be determined, as each gives specific information on the sequence under examination.

A final consideration with z-scores involves those of excessive magnitudes. From the comments following (4.4), regarding how very regular sequences can be extreme outliers relative to the Gaussian, and the remarks on the divergent z-scores of periodic sequences in Section 9.6, the z-scores for some sequences can grow in proportion to sequence length. This is undesirable if we want to make meaningful comparisons between such distribution outliers at different product lengths, or different $p_{i}$, and even different alphabets.

Two approaches are proposed to 'normalize' the z-scores in these cases. Both involve first computing the maximum possible z-score, $z_{\max }$, for a sequence of that length.

In the binary case, this is achieved by finding the z-score of the narrowest fully regular sequence under the given $p_{i}$ and $s$. By narrowest we mean the shift sequence of the line segment that occupies the lowest volume (this is the sequence that preserves the largest $S_{\alpha}$ product). These amount to the Christoffel word cycles of Section 3.

In the $k>2$ case, we estimate $z_{\max }$ by first finding all block rows that contain a minimal, $\tau$-period orbit (guaranteed to exist for all $R_{h}$ ). Such orbits correspond to the lowest-volume walks. We then choose one such minimal orbit from the block row with the largest $\left\|\sum_{i} S_{i}\right\|_{\text {en }}$ value. This gives the best chance of a minimal orbit with a maximal number of parallel orbits that fit within the bounding simplex, and a correspondingly high probability.

The first method of z-score normalization is then to simply divide the z-score by $z_{\max }$. This normalizes to values $\leq 1$, with a normalized value of 0 still corresponding to the center of the normal distribution. A disadvantage to this method is it flattens the z-scores for all sequences, including the vast majority that are not outliers, and for which the z-score accurately reflects the probability of occurrence. To preserve the bulk of the distribution then, the second method will be a hybrid. We'll leave z-scores in some reasonable range, $[-x, x]$ (say $x=4$ ) alone, but compresses those lying outside the range so that they lie in some standard interval:

$$
z_{n}= \begin{cases}z & \text { when } z \in[-x, x] \\ \operatorname{sgn}(z)\left(x+f\left(\frac{|z|-x}{z_{\max }-x}\right)\right) & \text { when } z \notin[-x, x]\end{cases}
$$


where $f$ is some appropriate monotone increasing and preferably convex function: $f(0)=0, f(1)=\xi$, and where $\xi$ is chosen so that $x+\xi$ is the maximum normalized z-score (e.g. if $x=4$, perhaps $\xi \approx 2$ ).

In addition to the z-scores, Section 8 gives the further option of computing the information-theoretic homogeneity entropy of a symbolic sequence. This may be done, for example, through use of maximum likelihood estimates, finding a best-fit parameter set, $\left\{\left\{p_{1}, \ldots, p_{k}\right\}, \delta, \rho\right\}$, as will be done with heart rate data in Section 10.2. This might be considered a more regularity-centric option among the usual entropy-based sequence statistics, such as ApEn and SampEn. While ApEn and SampEn are well-suited for general measures of intra-sequence regularity, or selfsimilarity, the homogeneity entropy can serve as a more specific, focused measure of the evenness in symbol distribution.

9.8. Contrasts with complexity measures. It's worth emphasizing, the zscores do not, strictly speaking, measure randomness. Assume a sufficiently low $\delta$, and that the sequences under study have a stable $p$-proportion of zeros to sequence length. Then:

A periodic sequence will, eventually, see its z-scores diverge from $0 ; \mathrm{z}$-scores can detect periodicity, but only for sufficiently long runs, where they approach periodicity detection with probability 1 (see Section 9.6).

Conversely, non-periodic sequences, including chaotic or random, may also have high z-scores. Consider a sequence with $p$ proportion $p=(n-1) / n, n=d_{0}+d_{1}$, $n$ sufficiently large, where for some $r<n$ at each $(j n)$ th spot in the sequence, $j=1,2, \ldots$, we place a 1 in $[j n-r, j n+r]$ with uniform probability $P=1 /(2 r+1)$; all the remaining spots in the sequence will be 0 . Provided $r$ is sufficiently small, such a sequence will likely still have a very high z-score, even though it is obviously non-periodic and very random, though on a small scale.

From a programmatic complexity standpoint, high z-scores are an accurate measure of fewer fundamental operations (pads or splices) to make a sequence fully regular, and, conversely, lower z-scores require more numerous fundamental operations to achieve full regularity. The z-scores may be a poor test of other forms of programmatic complexity.

ApEn, SampEn, and other broad-based entropic methods are better suited for testing for general complexity; the regularity z-scores and the homogeneity entropy look fairly narrowly at their own notion of regularity with respect to the given fully regular sequence templates.

\section{Applications}

In this section, some applications will be explored.

10.1. Encoding a time series. Regularity analysis for time series typically involves everything from basic statistical measures such as variance, and on to nonlinear complexity-based measures such as Lempel-Ziv, and Kolmogorov entropy and its derivatives such as Approximate Entropy [42], and Sample Entropy [47]. These last are both simplified forms of Kolmogorov-Sinai entropy [27, 50], and involve measuring degrees of self-similarity within the signal. They have applications in numerous areas, including data mining [22], DNA analysis [19], finance [44], and biological signal analysis [43, 47, 63]. 
There are also more mundane methods, such as geometric measures, which plot for instance a histogram of consecutive time step differences. Shape-fitting is then performed, the particular shape providing information on the signal [37]. There are also frequency domain methods, such as the FFT, and more complicated frequency analysis models that may be tuned with additional parameters [37]. Note however that these methods can fail to capture obvious differences in signals-i.e. large evident differences in signals may be observed in spite of having the same variance, RMSSD, and even power spectra (see [37], Fig. 6). One common area of application is measuring the regularity/irregularity of human heart rates and other physiological signals [37, 42, 47].

Keeping in mind the strengths and weaknesses explored in Section 9, we'll apply z-score regularity analysis to two different time series: human heart rate data and the logistic map. For the heart rate data we'll also calculate the homogeneity entropy. This is accomplished simply, through use of maximum likelihood estimates.

It is straightforward to encode a discrete time series for z-score analysis using the binary sequence distribution. The inter-1 distance in 'sparse' bit strings, those having a high proportion of 0 s, can be used to encode signal amplitude. We consider $p=(n-1) / n, d_{0}=1$, for some $n$ sufficiently large. The z-scores will then measure the degree of deviation from a constant signal. The following is a procedure for comparing a set of signals, $Q_{1}, Q_{2}, \ldots$, with $t$ the horizontal time axis, and $y$ the vertical amplitude axis:

Pre-processing:

- trim each signals' outliers (say beyond $4 \sigma$ )

- compute each signals' average amplitude, $\mu_{a_{j}}$; then compute the max distance below the average for each, $d_{-\mu_{j}}=\mu_{a_{j}}-\min \left(Q_{j}\right)$; the signals will be offset so their averages are in the middle of a fixed vertical window, and the window positioned so that no signal's amplitude is $<0$

- take as the basic window height, $w_{h}$, the largest min/max spread of all the (trimmed) signals

- choose a position for the middle of the window, $w_{m}$, so that $w_{m}-d_{-\mu_{j}}>0$ for all $j$; it is also advised to position the window so that the majority of the offset signals' values will fall roughly in $y \in\left[w_{h}, 2 w_{h}\right]$ (this amounts to offsetting the lower edge of the window to $y=w_{h}$ ); this helps avoid extrema in signal bit separations (avoiding 1s too close together or too far apart; this is to avoid any asymmetries in z-score weighting between superand subregular covers, asymmetries for example between weighting $110 \ldots 0$ vs. $10 \ldots 01$, each sequence of length $2 n+1)$; the more restrictive of the two conditions may be chosen

- uniformly shift each signal $Q_{j}$ 's amplitudes so that their average amplitude, $\mu_{a_{j}}$, lies in the middle of the window (the $Q_{j}$ 's amplitudes will have $w_{m}-\mu_{a_{j}}$ added to their values)

Creating the binary:

- choose a matrix size resolution, $n$; the larger the matrix, the better the resolution, but the more computationally intensive the z-score calculations (both in computing matrix products, and also in determining the Lyapunov exponent, the variance, and the correction factor-though these last need only be computed once)

- discretize the spatial $(y)$ resolution to units of size $u=w_{m} / n$ 
- for each time step, given post-processed amplitude $a_{i}$, assign the binary segment $0 \ldots 01$ such that the total segment length is equal to $\left[a_{i} / u\right]$; the length of the segment encodes the amplitudes; by construction, regardless of the signal the segment length should always be greater than 1

Converting the binary to a matrix product:

- using $p=(n-1) / n, d_{0}=1, d_{1}=n-1$, and some suitable $\delta$ (e.g. $\delta=0.1$, compute the matrix products under the three main methods, their norms, and then the z-scores

10.2. Heart rate data: z-scores and entropy of homogeneity. Heart rate data, often in terms of inter-beat, or R-R intervals, is a frequent subject of regularity analysis. It can assist in disease and cardiac anomaly detection, and was the original context for the development of approximate entropy. For a brief but general discussion of considerations on measuring complexity and regularity in physiologic signals, see [17], and [38]. To apply z-score regularity measures to heart rate data, we proceed as for a time series. The z-score analysis will give us a precise idea of how much a given heart rate time series differs from an unvarying signal-i.e. each heartbeat the same time interval from the one before it. We note that the z-score measures presented here have an advantage over other heart rate variability measures in their independence from sample length (up to some minimal recording period for the central limit theorem to take effect).

A suitable matrix size resolution is chosen. We'll choose $n=50, d_{0}=1, d_{1}=$ 49 , so that $p=49 / 50$. The parameter estimates used for z-score computation were $\lambda=-0.67820, \sigma^{2}=28.407, \xi=50.5$, for splice-only ( $\xi$ is the correction factor; see Section 4.3); $\lambda=-0.69450, \sigma^{2}=71.738, \xi=20.0$, for pad-only; and $\lambda=-0.061307, \sigma^{2}=0.30683, \xi=6.0$, for pad-and-splice.

Assume a series of inter-beat intervals under some acceptable unit of measure; in these examples, we'll use seconds.

Compute the average R-R interval, $\mu_{R-R}$, for the series. The basic time unit is then $u_{t}=\mu_{R-R} / n=\mu_{R-R} / 50$. The cumulative sum of the $\mathrm{R}-\mathrm{R}$ sequence is computed, giving a sequence of time values at which a heartbeat occurs, $\left\{t_{j}\right\}_{j=1, \ldots, j_{f}}$. This is converted into a binary, of length $\left\lfloor t_{j_{f}} / u_{t}\right\rfloor+1$, by placing a 1 at positions $\left\lfloor t_{j} / u_{t}\right\rfloor+1$, and 0 s everywhere else. Each 1-bit will represent a heartbeat; the average inter- 1 distance, in bits, has been calibrated to be $n=50$. Note this in a sense normalizes the sequence to the average heart rate for a given subject; the z-scores are only looking at variations around the average R-R interval.

One notable characteristic of heart rates is that as a person ages, their heart rate tends to become less variable [49]. One test of a regularity statistic is to check whether it produces the expected differences when comparing the heartbeat data of young vs. old cohorts. The Fantasia heart rate dataset (available through Physionet: https://physionet.org/content/fantasia/1.0.0/; [24, 16]), was created by dividing subjects into a young cohort and an older cohort, and recording their heartbeats as they watched the Disney movie Fantasia.

Shown in Figure 23 are the results of R-R interval analysis on ten subjects of the Fantasia heart rate study, five young (1-5) and five old (6-10), each sampled over approximately 2000 heartbeats. The sequence statistics include SDNN, the standard deviation of the R-R intervals (time between heartbeats), in seconds; pNN50, the number of R-R intervals that differ from the previous by $>50 m S$, 

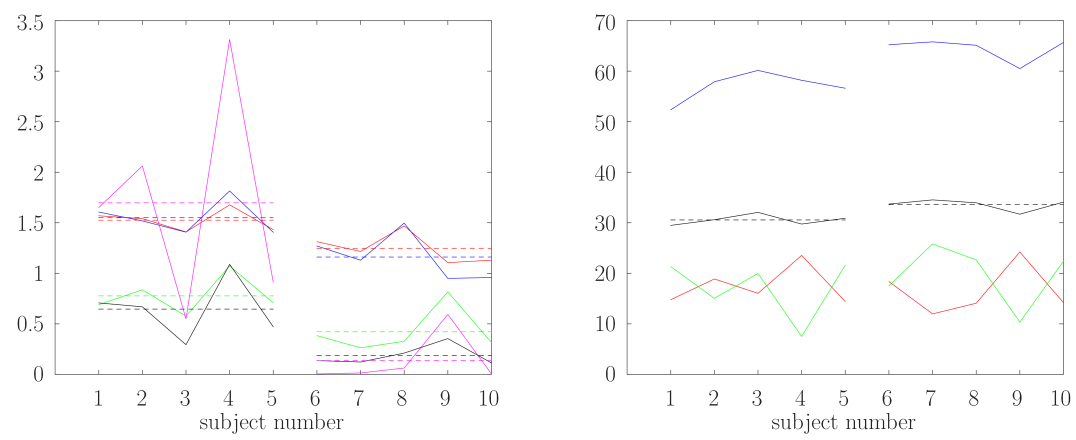

FiguRE 23. (color online) sequence statistics (left) and z-scores (right) for ten Fantasia subjects, five younger- (1-5) and five olderaged subjects (6-10); at left: $r=A p E n ; \quad b=S a m p E n ; g=S D N N$; $m=p N N 50 ;$ black $=r M S S D$; at right: $\mathrm{r}=$ splice-only; $\mathrm{g}=$ pad-only; $\mathrm{b}=$ pad-and-splice; black=average $\mathrm{z}$-score

divided by the total number of intervals; rMSSD, the root mean square of successive interval differences, computed by $\sqrt{\left(\sum_{i} I_{i+1}-I_{i}\right) /(N-1)}$, where the $N$ total R-R intervals (in milliseconds) are denoted by $I_{i}$. The two non-linear statistics computed are ApEn and SampEn. Both ApEn and SampEn utilized a filtering level of $r=$ $0.2 \sigma$, where $\sigma$ is the standard deviation of the set of R-R intervals. The SDNN, pNN50, and rMSSD have been rescaled to fit within the plot window. Dashed lines indicate the respective statistic's average over all in the young/old cohort.

For the usual sequence statistics, all the averages are lower in the older cohort, indicating less variability (in the SDNN, pNN50, and rMSSD cases), or less entropy (in the ApEn, and SampEn cases). (Note, some caution is needed in comparisons between the usual sequence statistics and the entropy measures, since the first weigh absolute differences in the R-R intervals, while ApEn and SampEn employ a relative, self-similarity-based cutoff, tailored to the R-R intervals' standard deviation.)

In the z-score plot, recall the higher the z-score, the more regular the sequence. There is little to differentiate the pad-only, and splice-only scores between cohorts, but pad-and-splice shows a noticeable difference, what contributes largely to the differences in the z-score averages. The averages are generally lower for the younger cohort, as expected. The individual exception is between subjects 3 (younger cohort) and 9 (older cohort). As with the traditional sequence statistics, the regularity z-scores for this pair are opposite expectations; the pad-and-splice scores are just barely the reverse of expectations for these subjects, while the z-score average has a more pronounced reversal.

Following the work of Section 8, the homogeneity entropy for the heartbeat data set will also be estimated. This will be accomplished with maximum likelihood estimates: finding the best-fit parameter set, $\left\{\left\{p_{1}, \ldots, p_{k}\right\}, \delta, \rho\right\}$, under pad-andsplice. As with z-score analysis, this benefits from not being dependent on sample sequence length, beyond some minimal length required to ensure accuracy of the entropy rate, as discussed in Section 8.2, and accuracy in the maximum likelihood estimate. 

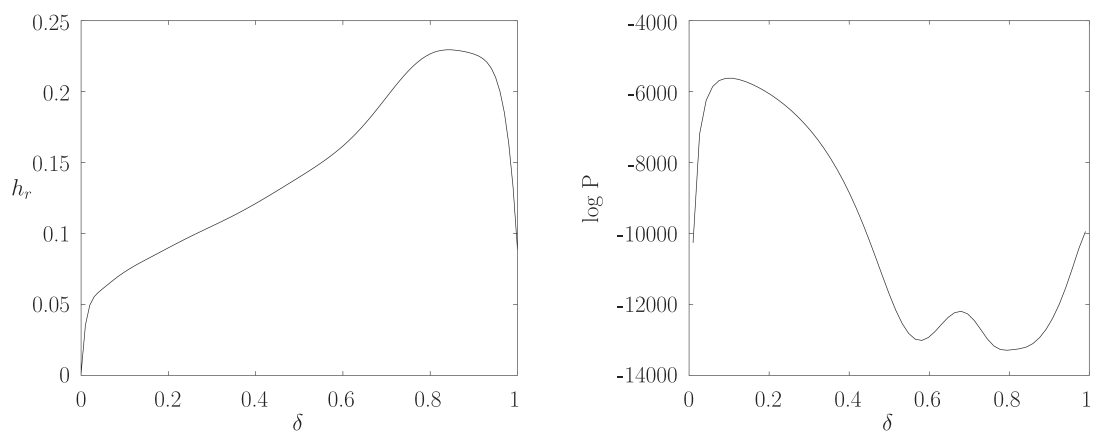

FigURE 24. on left: estimated entropy rates for the regularity distribution $p=49 / 50, s=50, \delta \in[0,1]$, pad-and-splice; on right: an example of obtaining a maximum likelihood estimate, this for heart study subject 9 (older cohort)-the maximum (log) probability occurs at $\delta \approx 0.1003(s=50)$, what corresponds to $h_{r} \approx 0.07305$ by the plot on the left

Given the heartbeat sequences are modeled by a $p=49 / 50$ distribution, it remains to estimate the other two parameters: the value of $\delta$, and the proportion spread (matrix dimension).

By the nature of heartbeats, the encoded binary sequence should be within a few edits of the regular $\overline{10 \ldots 0}$ template sequences throughout. This suggests that a model restricted to the lowest matrix dimension, $s=50$, would suffice. A more general approach is to admit potential models over the entire region $s \geq 50$.

For purposes of illustration we've chosen the second. Though with sources this homogeneous, the extension to models at $s>50$ is less critical. In fact, in spite of some of the subjects' best fitting a model at $s=51$, very little difference was found in entropy scores between the looser, $s \geq 50$, model, and the more limited $s=50$ model.

Specifically then, given binary R-R interval sequence $b_{n}$, a general maximum likelihood estimate involves computing the matrix product norm probabilities over all $\delta \in[0,1]$, and all 'reasonable' matrix dimensions $s \geq 50$. The $\{\delta, s\}$ parameter set with the highest probability is retained. The entropy rate $h_{r}$ for the parameter set is then computed, and recorded as the estimated homogeneity entropy of $b_{n}$.

For the Fantasia subjects, the maximum likelihoods occurred at matrix dimension $s=50$ for subjects 4 and $6-10$, while for subjects $1-3$ and 5 (all in the young cohort), the maximum likelihood occurred at $s=51$.

Figure 24, on the left, shows the entropy rate estimates for $p=49 / 50, s=50$, $\delta \in[0,1]$, under pad-and-splice. On the right is an example plot of the log of the probability of subject No. 9's binary R-R interval sequence as a function of $\delta$, computed as usual from the associated matrix products: $\log \left(c_{N}\left\|Q V_{m} Q \cdots V_{1} Q\right\|_{e n}\right)$. It's maximum, at around $\delta \approx 0.1003$, suggests a maximum likelihood parameter set of $\left\{\left\{p_{0}, p_{1}\right\}, \delta, s\right\}=\{\{49 / 50,1 / 50\}, 0.1003,50\}$. The associated entropy rate is approximately 0.07305 . The procedure is repeated for the other nine subjects.

Figure 25 shows the homogeneity entropy estimates for the Fantasia heart rate subjects. The ordering is, left to right, 1 through 5 for the younger subjects, and 


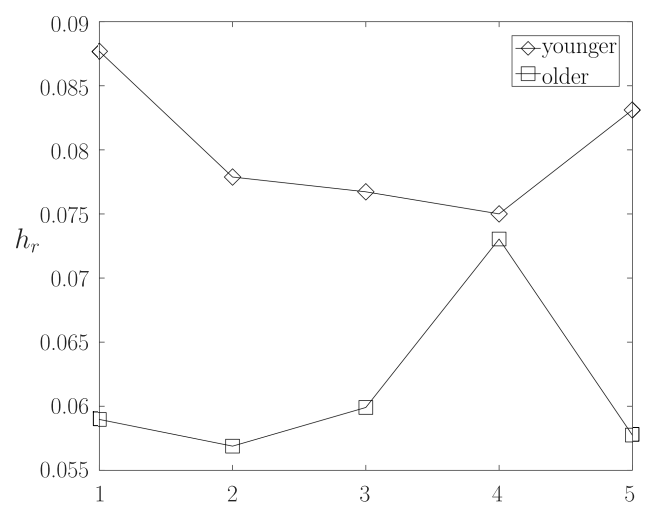

FIGURE 25. homogeneity entropy estimates for the ten Fantasia heart rate study subjects; the older cohort has been renumbered $6 \rightarrow$ $1,7 \rightarrow 2, \ldots, 10 \rightarrow 5$

6 through 10 for the older subjects. Unlike the ordinary sequence statistics, which featured subjects 3 and 9 as closest in variability, the homogeneity entropy puts subjects 4 and 9 the closest. Overall, this scoring method entirely separates the young and old cohorts into their expected regions (higher entropy for the younger, and lower entropy for the older), but for the closest to only $\approx 3 \%$.

10.3. The logistic map. In this section, z-score analysis will be applied to a true time series, using the encoding procedure outlined in Section 10.1. We'll look at the output of the logistic map at two different values of $r, 3.67$ and 3.87, with initial $x_{0}=0.5$. The sequence of amplitudes will be iterations 1001 to 1500 . Since the logistic map in these ranges stays within $[0,1]$, we'll choose a vertical window offset of 1 , putting the oscillations in the range $[1,2]$. The signal amplitudes in each case are shifted to place their average in the middle of the window.

For encoding, we'll use $\delta=0.1$, and the resolution parameter (matrix size) $n=50$, and $d_{0}=1, d_{1}=49$.

To build up a picture of the time series' regularity characteristics, it will be checked for regularity on different scales, or wavelengths. For any given wavelength, $L=1,2, \ldots$, and phase $\phi \in[1, L]$, the points $\phi, L+\phi, 2 L+\phi, \ldots$ will be sampled. The resulting subsequence is converted to a binary, as in Section 10.1, then its average z-score is determined. The average z-score over all phases at that wavelength is computed and retained.

Since the number of samples will differ between different sampling wavelengths, to compare results between wavelengths, we normalize the z-scores so their maximum is 1 (as explained in Section 9.7).

Figure 26 shows the two plots. The top plots are iterations 1001 through 1250 of the logistic map at the indicated $r$ and starting from $x_{0}$. The bottom plots are the average z-scores, averaged over all phases, at wavelengths $L \in[1,10]$ (indicated on the bottom axis).

All the z-scores will skew high because of the vertical window configuration; the average inter- 1 bit distance in the binary encoding will be 50 , and by window design, it will not go below $\approx 33$, nor above $\approx 66$. 

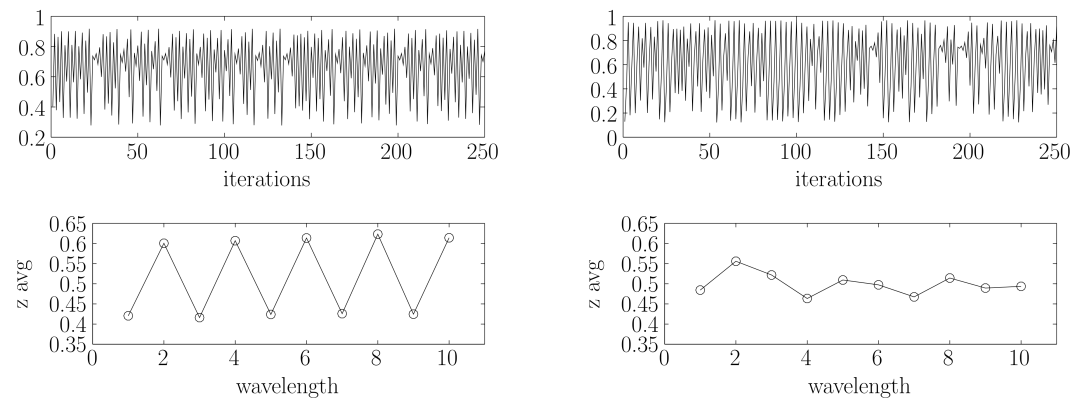

FigURE 26. plots for logistic map at $r=3.67$ (left) and $r=3.87$ (right), both at $x_{0}=0.5$, for iterations in $[1001,1250]$; the $z$ score plots (bottom) are derived from the sequence of iterations [1001,1500] (500 samples total) and are the averages of the three $z$-score averages at each phase for a given wavelength

Note that in general a high z-score suggests close-fit to a template, but a low z-score may fail to differentiate between random, and periodic or quasi-periodic sequences that are a poor fit to the template. This is illustrated in the plots at either $r$ value; the relatively lower z-scores at some wavelengths don't really reflect randomness in the sequence (it is quite periodic), but result from regular oscillations that are a poor fit to the expected fully regular $\overline{10 \ldots 0}$ template (a 1 followed by 49 0 s, and cyclic rotations).

The peaks at even values of $L$ in the $r=3.67$ plot reflect the relatively strong 2-periodicity of the logistic map's oscillations at this $r$, in spite of the varying amplitudes. (Note this does not have the advantages of, say, a Fourier spectrum: we don't have orthogonality, and a strong regularity at wavelength $L_{1}$ will likely show up again at $k L_{1}, k>1$.)

At $r=3.87$, an up-close view of the logistic map (not shown) indicates alternating $2-$ and 3 -period oscillations; i.e. concatenations of roughly $2-$ period stretches alongside 3 -period stretches. This is reflected in the z-score spectral plot, as the values at wavelengths 2 and 3 are relatively higher.

10.4. Sinai billiards. Here we apply the regularity measure to an ergodic dynamical system. For ergodic system $(X, \Sigma, \mu, \phi)$, with probability space $(X, \Sigma, \mu)$, and $\phi$-invariant, ergodic measure $\mu$, we know by ergodicity that almost all trajectories must have access to all regions of positive measure within the phase space, $X$, infinitely often. Regularity analysis provides a way to examine details of this behavior. We partition the phase space into disjoint regions, $\left\{P_{1}, \ldots, P_{k}\right\}, \sum_{i} \mu\left(P_{i}\right)=1$, and encode the dynamics of a trajectory via partition-based symbol assignments (discretizing time if necessary). We then apply regularity analysis to the symbolic sequences generated by the trajectory, starting at some $x_{0}$. Symbol $\alpha_{i}$ has expected proportion $p_{i}=\mu\left(P_{i}\right)$, and the z-score analysis is applied accordingly, at some level of proportion spread.

In the area of mathematical billiards, the Sinai billiard is a well-known example of a system that is not only ergodic, but a Bernoulli system [52, 11]. It relies on the presence of a central disc that acts as a scatterer on trajectories. 


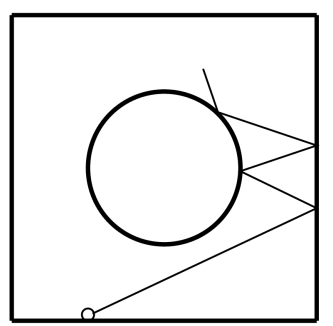

Figure

27. Sinai

billiards; the

central disc acts

as a scatterer
Here we generate an image plot corresponding to different trajectories in the Sinai billiard. To create the plot, the square billiard table $[0,1]^{2}$ was partitioned into four equal quadrants, $x<>1 / 2, y<>1 / 2$, each quadrant assigned a symbol. An arbitrary initial point of $(0.25,0.001)$ was chosen along the bottom wall. For initial angles in the range $[0, \pi]$ and unit velocity, and central disc radii in the range $[0,0.25]$ the trajectory of the particle was tracked over 300 time units (amounting to a total path length of $s=300$, or around 300 collisions with the boundaries). The Julia programming language's DynamicalBilliards.jl package [4] was used to generate the data. At every unit time, the trajectory's position was sampled, and its quadrant recorded. This produced a four-symbol sequence of length $\approx 300$. The symbol sequences' z-scores were then computed, using the four-symbol equiprobable $S_{i}$ templates under pad-and-splice.

Shown in Figure 28 is an image plot of the z-scores as a function of disc size and initial angle. The vertical streamers starting at the bottom are the result of regularity extremes from periodic or near-periodic behavior of the orbits. The central bolus and its main vertical streamer near $\pi / 2$ reveal the periodic orbits that bounce between the top and bottom walls along or near the line $x=0.25$. The streamers near 0 and $\pi$ are similar, showing bouncing between left and right walls, near the x-axis. Other streamers result from periodicities of the particle bouncing off all four walls. Two-walled bounces create two-symbol strings, which get very low z-scores; four-walled bounces tend to cover all four quadrants, and receive very high z-scores. As the central disc grows larger $(\sqrt{r}$ increases $)$, it has a greater scattering effect on the trajectories, as well as producing 'bottlenecks': the particle will have a harder time transitioning from one quadrant to the other, having to squeeze between a wall and the disc. The net effect is that the periodic and nearperiodic behavior starts to dwindle, and the overall regularity of the sequence is diminished; this is seen in the truncation of the streamers, and the darker colors (reflecting lower z-scores) as $\sqrt{r}$ increases.

\section{Discussion}

We have seen the development of a class of regularity-based probability distributions over sequences with alphabet $\mathcal{A},|\mathcal{A}|=k$, constructed around matrix product norms. The distributions were parametrized by the set $\left\{\left\{p_{1}, \ldots, p_{k}\right\}, \delta, \rho\right\}$, with $p_{i}$ the symbol probabilities. The $\delta$ parameter mediated between a topological and Bernoulli randomness, and $\rho$ was the proportion spread, what amounted to the allowed local variation in symbol density. The distribution of matrix norms, in turn, were seen to obey a central limit theorem. The distributions further paralleled an ergodic stochastic process, allowing a well-defined entropy rate, and, in the single block row case, satisfied an AEP. The existence of an AEP offers a calculable, and theoretically achievable compression limit for any sequence successfully modeled by such sources. At the core of the regularity score assignment was a process of 


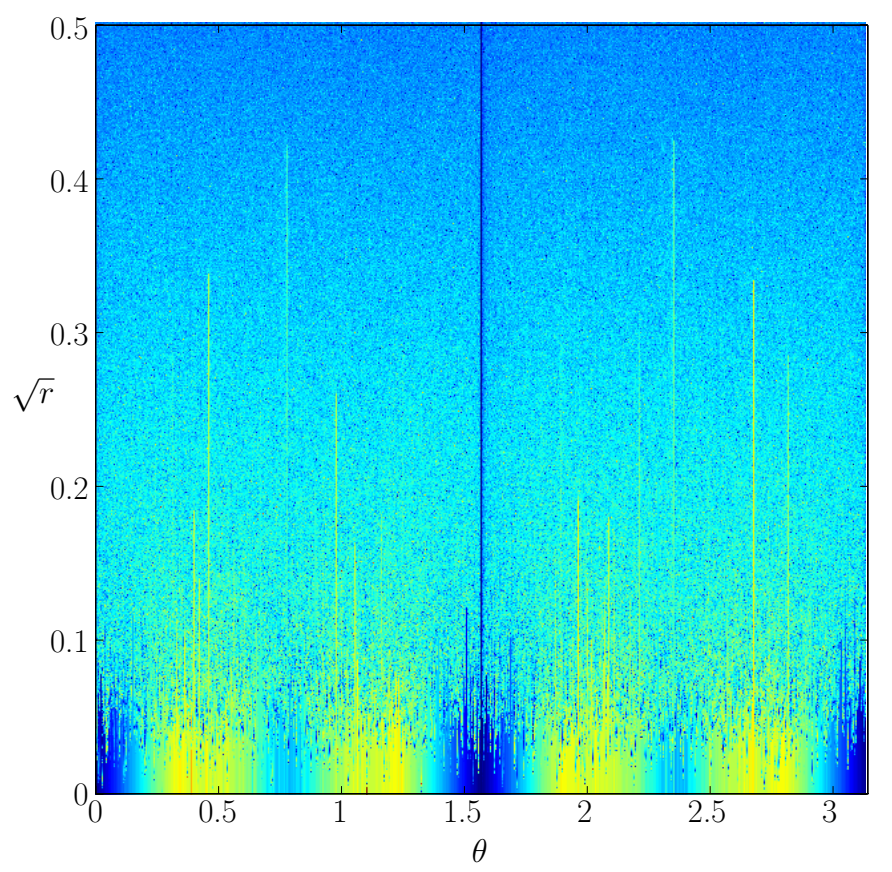

FiguRE 28. (color online) from regularity to irregularity; $z$-score image plot for Sinai billiard of side length 1 and central disc diameter $\in[0.0 .5]$; initial point is $(0.25,0.001)$, with unit velocity and initial angle $\theta$, tracked over a path length of 300 ; z-scores range from $\approx-11.2$ to $\approx 26.1$; warmer colors correspond to higher values; $\sqrt{r}$ scaling used to emphasize low-r behavior

symbol insertions and deletions, much like an aggregation of individual Levenshtein distances, performed on all scales relative to the template sequences. All of this fit naturally within the matrix structure.

It was suggested in Section 9 that the measures are sufficiently different from ordinary statistical variance to be of interest. For one, the matrix-based measure is inherently order dependent, owing to the non-commutativity of matrix products, while variance is not. In addition, the underlying structure, the padding and splicing dynamic, is of course fundamentally different from the procedure for computing variance, so we expect different behavior. This expectation is reinforced specifically in Sections 9.2 and 9.4, where although we saw correlations between intuitive notions of variance and the regularity measure, they are not nearly total. It would also be difficult to construct a simple variance measure that would allow meaningful regularity comparisons between sequences of different lengths, or those of different symbol proportions, or proportion spread. The matrix method accommodates all this in a single compact form.

It's worth emphasizing that in spite of the flexibility of the distributions, they are, on the face of it, not well-suited to model sequences such as $\overline{1111100000}$. This 
is obviously best modeled with $p=1 / 2$, but the proportion spread parameter, $\rho$, eludes quantification. Leaving the matrix dimension minimal $(s=2)$ would render this sequence highly improbable under that parameter set, while raising the matrix dimension to admit this sequence as a template sequence (to $s=6$ ) would admit a large set of other sequences with large proportion spread, and consequently fail to capture the sequence's simplicity. The remedy in this case is to window the sequence at a wavelength of 5 , an approach explored with the logistic map in Section 10.3.

Another potential drawback is that the z-scores can grow without bound, certainly for periodic sequences (Section 9.6). We've addressed this with the either one- or two-stage re-normalization process (Section 9.7).

The generation of the template sequences on alphabets of more than two symbols may in and of itself may be of interest, in that it presents a compact way, via the $S_{\alpha}$ matrices and their associated graphs, of representing all possible regular infinite sequences with a given proportion spread. At the minimal proportion spread, the template sequences offer a natural geometric extension of Christoffel words. We've also seen a potential application in ergodic systems, allowing a granular examination of the dynamics in terms of the homogeneity of trajectories through the phase space. This goes beyond what we may learn, for instance, by sorting the system along the ergodic hierarchy.

Overall, we have shown two, separate but highly related methods for qualifying the regularity of a symbolic sequence. Z-score analysis allows checking a given sequence for its degree of regularity against a fixed $\left\{\left\{p_{1}, \ldots, p_{k}\right\}, \delta, \rho\right\}$ template set. This might be useful for measuring the degree of regularity of a sequence or set of sequences under a sweep of $p_{i}$ or other parameters, as was done with the logistic map in Section 10.3. A disadvantage to z-scores is that they measure a sequence's distance away from the mean regularity of the most numerous sequences, this when the majority of probability mass from a pure $\left\{\left\{p_{1}, \ldots, p_{k}\right\}, \delta, \rho\right\}$ regularity source may lie high in the Gaussian tail, within a rare set of 'typical' sequences. If we are measuring sequences that are typical of such a source, the z-scores suffer from an associated normalization problem. Homogeneity entropy, on the other hand, is well-suited to sequences known to originate from a pure, parametrized regularity source. It also has the advantage of offering an information-theoretic classification of the test sequence. But, for at least one drawback, homogeneity entropy offers no relative measure of regularity between sequences deemed to originate from the same source. Consider, by the AEP, in the single block row case, that the probability of sequence $s$ is asymptotically assured of satisfying $-\log (P(s))=m h_{r}+\mathcal{O}\left(m^{1-\epsilon}\right)$, where $h_{r}$ is the entropy rate. Given that the variance under the central limit theorem is $\mathcal{O}(\sqrt{m})$, this permits a fair amount of regularity spread within a set of 'typical' sequences, what a plain homogeneity entropy measurement via maximum likelihood would miss. In this case, any degeneracy among the set of 'typical' sequences under study could be measured via z-score analysis, with the caveat the re-normalization process of Section 9.7 may highly compress z-score differences far in either tail of the CLT Gaussian.

Some other drawbacks and limitations of the method include the rather large matrix sizes required for longer symbol alphabets, and/or rational symbol proportions with reduced fractions of large denominators (Section 7.6). These can require large block-row matrices: matrix dimensions of order 1000 and higher even for sequences with just four symbols, and a correspondingly high demand on processing 
power. Another area of potential high demand is in estimating the homogeneity entropy of a sequence: both the computational costs of making the maximum likelihood estimate, which requires taking matrix products over possibly many values of the parameters, as well as the costs to performing the resampled Monte Carlo method of Section 8.3 to ensure a sufficiently accurate entropy rate. These latter values however could be computed and stored beforehand, as with the computations shown in the entropy level curves of Figure 18.

To overcome the difficulties with longer symbol alphabets, an approximate regularity measure may be created by treating the test sequence as a superposition of binary sequences, one for each symbol, as mentioned at the start of Section 7.7. A weighted average over all symbols, of either z-scores, or entropy rates, could then be taken as a composite measure of the sequence homogeneity. This though suffers from obvious inefficiencies, including from an entropy standpoint, since there is obviously much greater variability in the sum of binaries than in a single sequence with $k$ symbols. It may also produce distortions in the z-scores, since a sequence of $k>2$ symbols may well be maximally homogeneous in aggregate, but, because of symbol placement restrictions and inter-symbol competition the binary projection of one or more symbols, may not fit a purely homogeneous template for their respective projected parameter sets on two symbols. (See [28] for a detailed look at such placement restrictions.) Another option is to choose only one block row, or, further, only select graph routes within a given block row to construct a reduced $S_{\alpha}$ template set. Or, the simplex method may be discarded altogether, and the homogeneous $S_{\alpha}$ product templates may be constructed 'by hand,' from work such as [28] on minimizing symbolic sequence discrepancy. Note that the sequences rendered by the template matrices are all cyclic (ensuring, for instance, the central limit theorem applies), and any hand-made templates should fit this condition as well. 


\section{Appendix}

Following are the algorithms for generating the $S_{\alpha}$ matrices, in their final, condensed, block row form. For better memory utilization, all of these routines may be done in sparse matrix form.

inputs: $\left\{d_{1}, \ldots, d_{k}\right\}, h$

outputs: the $S_{\alpha}$ in block diagonal form; if $h$ is too small, output of reduction step will be null

First, generate the raw $S_{\alpha}$ :

$u=h(k-1)$

// $T$ will hold unit shear cells, in altitude coordinates

$T=$ all $k$-tuples $\left(w_{1}, \ldots, w_{k}\right), w_{i} \in[0, u-1], \sum_{i} w_{i}=u-1$

create $M: T \rightarrow[1, \operatorname{card}(T)] / /$ create one-to-one coordinate map

set each $S_{i}, i \in[1, k]$, to a zero $\operatorname{card}(T)$ by $\operatorname{card}(T)$ matrix

for $i=1$ to $k$ do

create shift vector $s_{i}=\left[-d_{1}, \ldots,(k-1) d_{i},-d_{i+1}, \ldots,-d_{k}\right]$

end for

for all $w \in T$ do

for $i=1$ to $k$ do

$v=w+s_{i}$

if $v$ is in bounds, save $k$-tuple pair $(v, w) \rightarrow U(i)$

$/ /(v$ is in bounds if all components are $\in[0, u-1])$

end for

end for

for $i=1$ to $k$ do

for 1 to $\operatorname{card}(U(i))$ do

set $S_{i}$ at $(M(v), M(w))$ equal to 1

end for

end for

Reduction step: pare the $S_{\alpha}$ of rows and columns of zeros.

$Q=\sum_{i} S_{i}$

$z c r=$ true $/ /$ there are columns of all zeros remaining

$z r r=$ true $/ /$ there are rows of all zeros remaining

while $z c r==$ true and $z r r==$ true and $Q$ is non-null do

$Z C=$ indices of the all-zero columns of $Q$

$Z R=$ indices of the all-zero rows of $Q$

$/ /$ remove rows/cols corresponding to zero rows/cols, and vice-versa:

for $i=1$ to $k$ do

remove all cols and rows indexed by $Z C$ from $S_{i}$

remove all rows and cols indexed by $Z R$ from $S_{i}$

end for

$Q=\sum_{i} S_{i}$

$z c r=i \operatorname{sempty}(Z C)$

$z r r=i \operatorname{sempty}(Z R)$

end while 
The last step finds all connected components within the pared $S_{\alpha}$. It performs a breadth-first search: starting with any node, it finds all exit nodes; these go in the 'covered' nodes list. Each exit node is then explored the same way, iteratively, giving another set of exit nodes. The algorithm ends when all exit nodes coincide with nodes already covered. Output will be one or more sets of binary matrices, $\{F(1), \ldots, F(k)\}$, forming a given block row.

// to_check, set of column indices being checked on current pass

// check_next, set of column indices to check on next pass

// checked_cols, set of all column indices that have been checked

$/ /$ for matrix $M, M(\#, c)=$ all coord pairs where $M$ has a 1 at $(r, c)$

while $S_{\alpha}$ are nonempty do

to_check $=\{1\}$

while to_check is nonempty do

for $j=1$ to size of to_check do

for $i=1$ to $k$ do

save $(r, c)$ values $S_{i}\left(\#, t_{\text {to_check }}(j)\right)$ in $Q(i)$

end for

check_next $=$ all $r$ 's in all $Q(i)$ 's not already in checked_cols

end for

include to_check in checked_cols

to_check $=$ check_next

end while

// condense the $(r, c)$ pairs in all the $Q(i)$ and save:

$R=$ set of all unique $r$ and $c$ values in all $Q(i)(r, c)$ pairs

create map $T: R \rightarrow[1, \operatorname{card}(R)]$

set each $F(i), i \in[1, k]$, to a zero $\operatorname{card}(R)$ by $\operatorname{card}(R)$ matrix

for $i=1$ to $k$ do

for all $(r, c)$ pairs in $Q(i)$ do

set $F(i)$ at $(T(r), T(c))$ equal to 1

end for

end for

save all $F(i)$ from this pass // the $F(i)$ will form a block row

// cleanup:

reduce the $S_{\alpha}$ by removing all columns in checked_cols, and assoc. rows

clear $Q$, checked_cols

end while

History of revisions (OSF postings):

Version 2 (Feb 2020): replaced the idea of 'sojourns' with Christoffel words for binary template sequences; various improvements in exposition

Version 3 (Jan 2021): correction in what is now Section 7.8, to state that for z-score analysis for longer symbol alphabets, whole matrix products must be taken (and not just the product of block rows corresponding to the largest Lyapunov exponent); the inclusion of cylinder graphs for representing template sequences on longer alphabets; substantial additions to the entropy section, what is now Section 8 , and inclusion of homogeneity entropy where appropriate throughout the paper; various other improvements in exposition 
Version 4 (May 2021): corrected inadvertent omission in entropy section-the multiple block row case, and have limited the AEP to single block row case; various improvements in exposition

\section{REFERENCES}

[1] Altman, E., Gaujal, B., Hordijk, A. Balanced sequences and optimal routing Journal of the ACM (JACM), Vol. 47, No. 4; pp. 752-775 1997.

[2] Bai, Z.Q. On the cycle expansion for the Lyapunov exponent of a product of random matrices J. Phys. A, Vol. 40, No. 29, pp 8315-8328 2007.

[3] Bárány, I. On the Power of Linear Dependencies In: Grötschel M., Katona G.O.H., Sági G. (eds), Building Bridges, Bolyai Society Mathematical Studies, vol 19. Springer, Berlin, Heidelberg 2008.

[4] Beazanson, J., Edelman, A., Karpinski, S., Shah, V. Julia: A Fresh Approach to Numerical Computing SIAM Review, Vol 59, No. 1, pp 65-98 2017.

[5] Ben-Naim, E., Krapivsky, P.L. Weak Disorder in Fibonacci Sequences Journal of Physics A: Mathematical and General, Vol. 39, No. 20, L301-L307 May 3, 2006.

[6] Benoist, Y., and Quint, J.-F. Central limit theorem for linear groups Ann. Probab. 44 (2), 1308-1340 2016.

[7] Benoist, Y., and Quint, J.-F. Random Walks on Reductive Groups Ergebnisse der Mathematik und ihrer Grenzgebiete. 3. Folge / A Series of Modern Surveys in Mathematics [Results in Mathematics and Related Areas. 3rd Series. A Series of Modern Surveys in Mathematics], vol. 62, Springer, Cham, 2016.

[8] Bougerol, P., Lacroix, J. Products of random matrices with applications to Schrödinger operators Progress in Probability and Statistics Vol. 8, Birkhäuser Boston, Boston, MA 1985.

[9] Charbonneau, P., Li, Y., Pfister, H., Yaida, S. Cycle-expansion method for the Lyapunov exponent, susceptibility, and higher moments Physical Review E 96, 032129 September 2017.

[10] Chassaing, P., Letac, G., Mora, M. Brocot sequences and random walks in SL(2,R) In: Heyer H. (eds) Probability Measures on Groups VII, Vol. 1064, pp 36-48 Springer, Berlin/Heidelberg, 1984.

[11] Chernov, N., Makarian, R. Introduction to the Ergodic Theory of Chaotic Billiards, 2nd ed. Instituto Nacional de Matematica Pura e Aplicada, Rio de Janeiro, 2003.

[12] Chernyatiev, A.L. Balanced Words and Dynamical Systems Journal of Mathematical Sciences, Volume 156, Issue 2, pp 351-358, January 2009.

[13] Crisanti, A., Paladin, G., Vulpiani, A. Products of Random Matrices in Statistical Physics Springer: Berlin/Heidelberg, Germany 1993.

[14] Droubay, X., Justin, J., Pirillo, G. Epi-Sturmian words and some constructions of de Luca and Rauzy Theoret. Comput. Sci. 255(1-2), pp. 539-553, 2001.

[15] Furstenberg, H., Kesten, H. Products of random matrices Annals of Mathematical Statistics Vol. 31, No. 2, pp. 457-469 1960.

[16] Goldberger AL, Amaral LAN, Glass L, Hausdorff JM, Ivanov PCh, Mark RG, Mietus JE, Moody GB, Peng C-K, Stanley HE PhysioBank, PhysioToolkit, and PhysioNet: Components of a New Research Resource for Complex Physiologic Signals Circulation. 101(23):e215-e220. 2003.

[17] Goldberger, A., Peng, C.-K., Lipsitz, L. What is physiologic complexity and how does it change with aging and disease? Neurobiology of Aging 23 23-26 2002.

[18] Guivarc'h, Y., Raugi, A. Frontière de Furstenberg, propriétés de contraction et théorèmes de convergence Z. Wahrsch. Verw. Gebiete 69 (2), 187-242 1985

[19] Gusev, V.D.; Nemytikova, L.A.; Chuzhanova, N.A. On the complexity measures of genetic sequences Bioinformatics, 15, 994-999, 1999.

[20] Graham, R., Knuth, D., Patashnik, O. Concrete mathematics: a foundation for computer science, 2nd Edition Addison-Wesley Professional, Boston MA 1994.

[21] Hajek, B. Extremal splittings of point processes Math. Oper. Res. 10, pp. 543-556, 1985.

[22] Holzinger, A.; Hörtenhuber, M.; Mayer, C.; Bachler, M.; Wassertheurer, S.; Pinho, A.J.; Koslicki, D. On entropy-based data mining. In Interactive Knowledge Discovery and Data Mining in Biomedical Informatics Springer: Berlin/Heidelberg, Germany; pp. 209-226, 2014.

[23] Hennion, H. Limit theorems for products of positive random matrices Annals of Probability Vol. 25, No. 4 (1997), pp. 1545-1587 1997. 
[24] Iyengar N, Peng C-K, Morin R, Goldberger AL, Lipsitz LA. Age-related alterations in the fractal scaling of cardiac interbeat interval dynamics Am J Physiol, 271:1078-1084 1996.

[25] Katok, A., Hasselblatt, B. Introduction to the Modern Theory of Dynamical Systems Cambridge University Press, New York 1995.

[26] Kleban, P, Özlük, A.E. A Farey Fraction Spin Chain Communications in Mathematical Physics Volume 203, Issue 3, pp 635-647 June, 1999.

[27] A.N Kolmogorov. A new metric invariant of transient dynamical systems and automorphisms in Lebesgue spaces Doklady Akademii Nauk SSSR, 119:768-771 1958.

[28] Kubiak, W. Fair Sequences In: Handbook of Scheduling Chapman and Hall/CRC, London/Boca Raton, 19-1 to 19-21 2004.

[29] Labbé, S., Reutenauer, C. A d-dimensional Extension of Christoffel Words Discrete Comput Geom 54, 152-181 2015.

[30] Le Page, E. Théorèmes limites pour les produits de matrices aléatoires Probability measures on groups (Oberwolfach, 1981), volume 928 of Lecture Notes in Math., pages 258-303. Springer, Berlin-New York 1982.

[31] Lima, R., Rahibe, M. Exact Lyapunov Exponent for Infinite Products of Random Matrices J Phys. A: Math. Gen. Vol. 27, No. 10, pp 3427-3437 1994.

[32] Lind, D., Marcus, B. Symbolic Dynamics and Coding Cambridge Univ. Press, Cambridge, U.K. 1995.

[33] Lorentzen, L., Waadeland, H. Continued Fractions, Vol. 1, 2nd Edition Atlantis Press, Paris, France 2008.

[34] Lyons, R. Singularity of Some Random Continued Fractions Journal of Theoretical Probability, Volume 13, Issue 2, pp 535-545 April, 2000.

[35] Mainieri, R. Cycle expansion for the Lyapunov exponent of a product of random matrices Chaos: An Interdisciplinary Journal of Nonlinear Science, Vol 2., Issue 11992.

[36] Mainieri, R. Zeta function for the Lyapunov exponent of a product of random matrices Physical Review Letters 68, 1965 March 1992.

[37] Malik M, Bigger JT, Camm AJ, Kleiger RE, Malliani A, Moss AJ, and Schwartz PJ Heart rate variability: standards of measurement, physiological interpretation, and clinical use Circulation 93: 1043-1065 1996.

[38] Mayer, C, Bachler, M, Hörtenhuber, M, Stocker, C, Holzinger, A, and Wassertheurer, S Selection of entropy-measure parameters for knowledge discovery in heart rate variability data BMC Bioinformatics; 15(Suppl 6): S2 Published online May 16, 2014.

[39] Meijer, H.G. On a distribution problem in finite sets Nederl. Akad. Wetensch. Indag. Math. 35 9-17, 1973.

[40] Morse, M., Hedlund, G. Symbolic Dynamics II. Sturmian Trajectories American Journal of Mathematics, Vol. 62, No. 1, pp. 1-42, 1940.

[41] Nielsen, J. L. Lyapunov exponents for products of random matrices unpublished project, Chaos: Classical and Quantum, Niels Bohr Institute 1997.

[42] Pincus, S. Approximate entropy as a measure of system complexity Proc. Natl. Acad. Sci. U.S.A. 88(6), 2297-301 March 15, 1991.

[43] Pincus SM, Viscarello RR Approximate entropy: a regularity measure for fetal heart rate analysis Obstet Gynecol. 79(2):249-55 February, 1992.

[44] Pincus SM Approximate Entropy as an Irregularity Measure for Financial Data Econometric Reviews, Taylor \& Francis Journals, Vol. 27 (4-6), pp. 329-362. 2008.

[45] Pincus, S. Strong Laws of Larger Numbers for Products of Random Matrices Transactions of the American Mathematical Society, Vol. 287, No. 1, pp. 65-89 January, 1985.

[46] Protasov, V. Yu, Voynov, A.S. Sets of nonnegative matrices without positive products Linear Algebra and Its Applications, 473: 749-765 2012.

[47] Richman, JS; Moorman, JR Physiological time-series analysis using approximate entropy and sample entropy American Journal of Physiology. Heart and Circulatory Physiology., 278 (6): H2039-49 June, 2000.

[48] Rosenstein, M., Collins, J., and De Luca, C. A practical method for calculating largest Lyapunov exponents from small data sets Physica D 65, Issue 1-2, pp. 117-134 May 15, 1993.

[49] Ryan, SM, Goldberger, AL, Pincus, SM, Mietus, J, Lipsitz, LA Gender- and age-related differences in heart rate dynamics: are women more complex than men? J Am Coll Cardiol.; 24(7):1700-7 December, 1994. 
[50] Sinai Ya.G. On the concept of entropy for a dynamic system Doklady Akademii Nauk SSSR, 124:768-771 1959.

[51] Sturman, R, Thiffeault, J Lyapunov Exponents for the Random Product of Two Shears Journal of Nonlinear Science, Volume 29, Issue 2, pp 593-620 April, 2019.

[52] Tabachnikov, S. Geometry and Billiards AMS, Student Mathematical Library, Vol. 30, USA, 2005.

[53] Tarsissi, L. Balance properties on Christoffel words and applications (Doctoral dissertation) Université Grenoble Alpes, 2017. retrieved from https://tel.archives-ouvertes.fr/tel01885914/document

[54] Tijdeman, R. On the distribution problem in finite and countable sets Journal of Combinatorial Theory, Series A, Vol 15, Issue 2, pp. 129-137 September, 1973.

[55] Tijdeman, R. The chairman assignment problem Discrete Mathematics, Volume 32 Issue 3, pp. 323-330 Elsevier Science Publishers B. V. Amsterdam, The Netherlands January, 1980.

[56] Trench, W. F. On the Eigenvalue Problem for Toeplitz Band Matrices Linear Algebra and Its Applications, Vol. 64, pp. 199-214 January 1985.

[57] Gaetano Valenza, Paolo Allegrini, Antonio Lanatà, and Enzo Pasquale Scilingo Dominant Lyapunov exponent and approximate entropy in heart rate variability during emotional visual elicitation Front Neuroeng. 2012; 5: 3. Published online February 29, 2012.

[58] Vanneste, J. Estimating generalized Lyapunov exponents for products of random matrices Physical Review E 81, 036701 March 2010.

[59] Viswanath, D. Random Fibonacci sequences and the number 1.13198824... Math. Comp. 69, No. 231, 1131-1155 2000.

[60] Vuillon, L. Balanced Words Bull. Belg. Math. Soc. 10, 787-805 2003.

[61] Waldspurger, C.A., Weihl, W.E. Stride Scheduling: Deterministic Proportional Share Resource Management Technical Memorandum MIT/LCS/TM-528, MIT Laboratory for Computer Science Cambridge, Massachusetts 1995.

[62] Watkins, J. C. "Limit theorems for products of random matrices" Random Matrices and Their Applications: Proceedings of the AMS-IMS-SIAM Joint Summer Research Conference June 17-23, 1984, edited by Joel E. Cohen, Harry Kesten, Charles Michael Newman, American Mathematical Society, Providence, R.I. 1986.

[63] Yentes, Jenna M.; Hunt, Nathaniel; Schmid, Kendra K.; Kaipust, Jeffrey P.; McGrath, Denise; and Stergiou, Nicholas The Appropriate Use of Approximate Entropy and Sample Entropy with Short Data Sets Annals of Biomedical Engineering, 41(2):349-65, February 2013.

[64] Yeomans, J. Optimal Level Schedules for Mixed-Model Just-in-time Assembly Systems (Doctoral dissertation) McMaster University, CA, 1992. retrieved from https://macsphere.mcmaster.ca/bitstream/11375/8675/1/fulltext.pdf

trevormwine@gmail.com (Georgia Tech Math alum) 